JOURNAL OF THE

AMERICAN MATHEMATICAL SOCIETY

Volume 24, Number 3, July 2011, Pages 603-648

S 0894-0347(2011)00691-7

Article electronically published on January 19, 2011

\title{
APOLLONIAN CIRCLE PACKINGS AND CLOSED HOROSPHERES ON HYPERBOLIC 3-MANIFOLDS
}

\author{
ALEX KONTOROVICH AND HEE OH, \\ WITH APPENDIX BY HEE OH AND NIMISH SHAH
}

\section{Contents}

1. Introduction

2. Reduction to orbital counting

3. Geometry of closed horospheres on $T^{1}\left(\Gamma \backslash \mathbb{H}^{3}\right)$

4. The base eigenfunction $\phi_{0}$

5. Spherical functions and spectral bounds

6. Equidistribution of expanding closed horospheres with respect to the Burger-Roblin measure

7. Orbital counting for a Kleinian group

8. The Selberg sieve and circles of prime curvature $\quad 636$

Acknowledgments

Appendix. Non-accumulation of expanding closed horospheres on singular tubes (by Hee Oh and Nimish Shah)

\section{INTRODUCTION}

A set of four mutually tangent circles in the plane with distinct points of tangency is called a Descartes configuration. Given a Descartes configuration, one can construct four new circles, each of which is tangent to three of the given ones. Continuing to repeatedly fill the interstices between mutually tangent circles with further tangent circles, we arrive at an infinite circle packing. It is called an Apollonian circle packing, after the great geometer Apollonius of Perga (262-190 BC).

See Figure 1 showing the first three generations of this procedure, where each circle is labeled with its curvature (that is, the reciprocal of its radius). Unlike the inner circles, the bounding circle is oriented so that its "outward" normal vector

Received by the editors January 20, 2009 and, in revised form, November 18, 2009, December 10, 2010.

2010 Mathematics Subject Classification. Primary 22E40.

Key words and phrases. Apollonian circle packing, Horospheres, Kleinian group.

The first author is supported by an NSF Postdoc, grant DMS 0802998.

The second author is partially supported by NSF grant DMS 0629322.

(C)2011 American Mathematical Society 


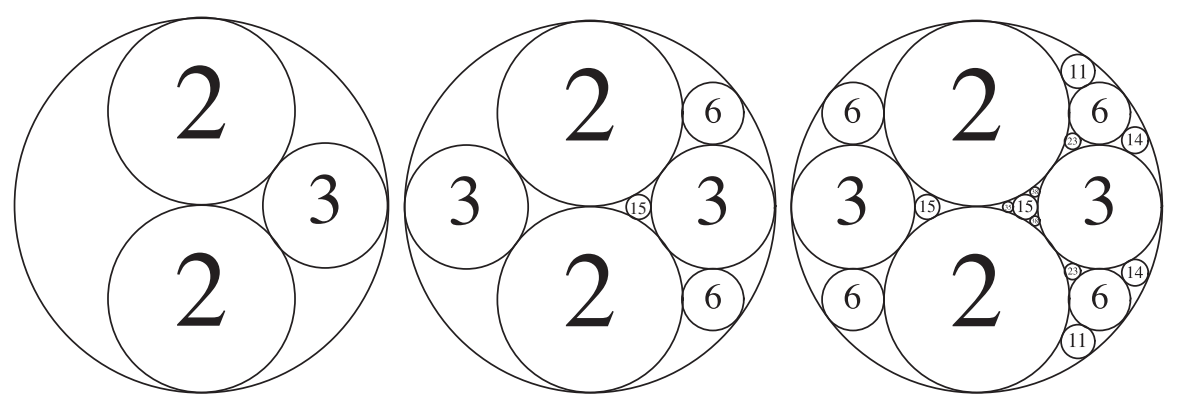

FiguRE 1. Generations 1-3

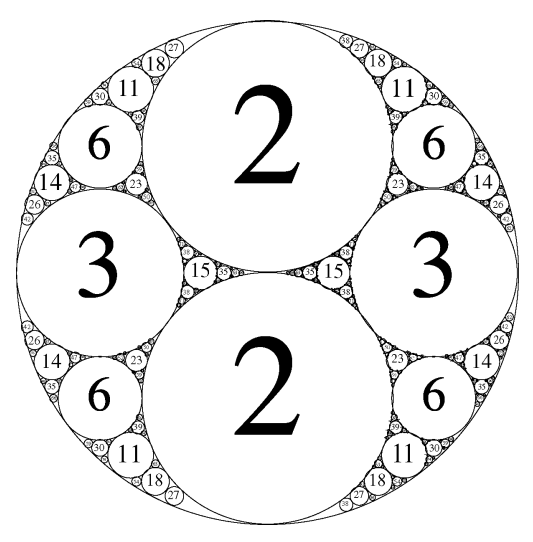

Figure 2. A bounded Apollonian circle packing

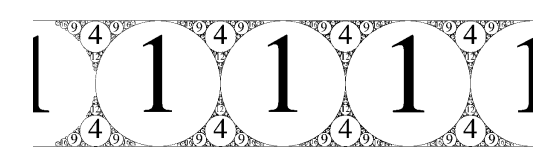

FiguRE 3. An Apollonian circle packing bounded by two parallel lines

points into the packing. In Figure 2 the outermost circle has curvature -1 (the sign conveys its orientation).

The astute reader would do well to peruse the lovely series of papers by Graham, Lagarias, Mallows, Wilks, and Yan on this beautiful topic, especially [16] and [15], as well as the recent letter of Sarnak to Lagarias [40] which inspired this paper.

Counting circles in an Apollonian packing: Let $\mathcal{P}$ be either a bounded Apollonian circle packing or an unbounded one which is congruent to the packing in Figure 3 .

For $\mathcal{P}$ bounded, denote by $N^{\mathcal{P}}(T)$ the number of circles in $\mathcal{P}$ in the packing whose curvature is at most $T$, i.e., whose radius is at least $1 / T$. For $\mathcal{P}$ congruent to the packing in Figure 3, one alters the definition of $N^{\mathcal{P}}(T)$ to count circles in a fixed period.

It is easy to see that $N^{\mathcal{P}}(T)$ is finite for any given $T>0$. The main goal of this paper is to obtain an asymptotic formula for $N^{\mathcal{P}}(T)$ as $T$ tends to infinity. To 


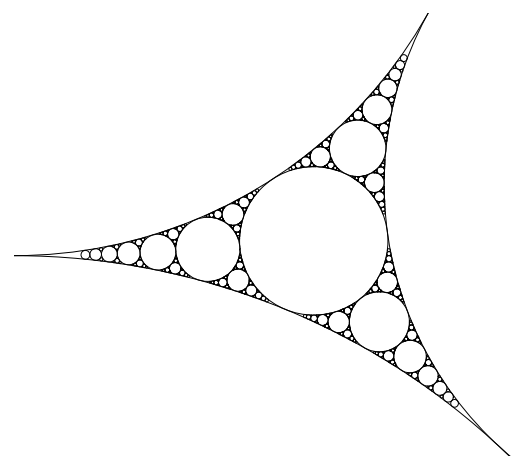

Figure 4. Circles in a curvilinear triangle

describe our results, recall that the residual set of $\mathcal{P}$ is defined to be the subset of the plane remaining after the removal of all of the interiors of circles in $\mathcal{P}$ (where the circles are oriented so that the interiors are disjoint). Let $\alpha=\alpha_{\mathcal{P}}$ denote the Hausdorff dimension of the residual set of $\mathcal{P}$. As any Apollonian packing can be moved to any other by a Möbius transformation, $\alpha$ does not depend on $\mathcal{P}$. The value of $\alpha$, though not known exactly, has been numerically computed to be $1.30568(8)$ by McMullen [29.

Boyd [6] showed in 1982 that

$$
\lim _{T \rightarrow \infty} \frac{\log N^{\mathcal{P}}(T)}{\log T}=\alpha .
$$

This confirmed Wilker's prediction [48, which was based on computer experiments.

Regarding an asymptotic formula for $N^{\mathcal{P}}(T)$, it was not clear from the literature whether one should conjecture a strictly polynomial growth rate. In fact, Boyd's numerical experiments led him to wonder whether "perhaps a relationship such as $N^{\mathcal{P}}(T) \sim c \cdot T^{\alpha}\left(\log \left(T / c^{\prime}\right)\right)^{\beta}$ might be more appropriate" (see [6], page 250).

In this paper, we show that $N^{\mathcal{P}}(T)$ has purely polynomial asymptotic growth. By $f(T) \sim g(T)$ with $T \rightarrow \infty$, we mean that $\lim _{T \rightarrow \infty} \frac{f(T)}{g(T)}=1$.

Theorem 1.1. Given an Apollonian circle packing $\mathcal{P}$ which is either bounded or congruent to Figure 3, there exists $c=c(\mathcal{P})>0$ such that as $T \rightarrow \infty$,

$$
N^{\mathcal{P}}(T) \sim c \cdot T^{\alpha} .
$$

In [6], Boyd actually considered the more general problem of counting those circles in a packing which are contained in a curvilinear triangle $\mathcal{R}$; let $N^{\mathcal{R}}(T)$ count the circles having curvature at most $T$ (see Figure 41). For this question, it does not matter whether or not the full packing is bounded; the counting function $N^{\mathcal{R}}(T)$ is always well defined. Since two such triangles are bi-Lipschitz equivalent, it follows from Theorem 1.1 that there exist constants $c_{1}, c_{2}>0$ such that for all $T \gg 1$,

$$
c_{1} \cdot T^{\alpha} \leq N^{\mathcal{R}}(T) \leq c_{2} \cdot T^{\alpha} .
$$

Though we believe that the asymptotic formula $N^{\mathcal{R}}(T) \sim c \cdot T^{\alpha}$ always holds, our techniques cannot yet establish this in full generality.

Primes and twin primes in an integral packing: A quadruple $(a, b, c, d)$ of the curvatures of four circles in a Descartes configuration is called a Descartes 
quadruple. The Descartes circle theorem (see e.g. 9]) states that any Descartes quadruple $(a, b, c, d)$ satisfies the quadratic equation 1

$$
a^{2}+b^{2}+c^{2}+d^{2}=\frac{1}{2}(a+b+c+d)^{2} .
$$

Given any three mutually tangent circles with distinct points of tangency and curvatures $a, b$ and $c$, there are exactly two circles which are tangent to all of the given ones, having curvatures $d$ and $d^{\prime}$, say. It easily follows from (1.2) that

$$
d+d^{\prime}=2(a+b+c) .
$$

In particular, this shows that if a Descartes quadruple $(a, b, c, d)$ corresponding to the initial four circles in the packing $\mathcal{P}$ is integral, then every circle in $\mathcal{P}$ also has integral curvature, as first observed by Soddy in 1936 [43]. Such a packing is called integral.

It is natural to inquire about the Diophantine properties of an integral Apollonian packing, such as how many circles in $\mathcal{P}$ have prime curvatures. By rescaling, we may assume that $\mathcal{P}$ is primitive; that is, the greatest common divisor of the curvatures is one. We call a circle prime if its curvature is a prime number. A pair of prime circles which are tangent to each other will be called twin prime circles. It is easy to see that a primitive integral packing is either bounded or the one pictured in Figure 3 ,

For $\mathcal{P}$ bounded, denote by $\pi^{\mathcal{P}}(T)$ the number of prime circles in $\mathcal{P}$ of curvature at most $T$, and by $\pi_{2}^{\mathcal{P}}(T)$ the number of twin prime circles in $\mathcal{P}$ of curvatures at most $T$. For $\mathcal{P}$ congruent to the packing in Figure 3 , one alters the definition of $\pi^{\mathcal{P}}(T)$ and $\pi_{2}^{\mathcal{P}}(T)$ to count prime circles in a fixed period.

Sarnak showed in [40] that there are infinitely many prime and twin prime circles in any primitive integral packing $\mathcal{P}$, and that

$$
\pi^{\mathcal{P}}(T) \gg \frac{T}{(\log T)^{3 / 2}} .
$$

Using the recent results of Bourgain, Gamburd and Sarnak in [4] and [5] on the uniform spectral gap property of Zariski dense subgroups of $\mathrm{SL}_{2}(\mathbb{Z}[i])$, together with the Selberg's upper bound sieve, we prove:

Theorem 1.4. Given a primitive integral Apollonian circle packing $\mathcal{P}$,

(1) $\pi^{\mathcal{P}}(T) \ll \frac{T^{\alpha}}{\log T} ;$

(2) $\pi_{2}^{\mathcal{P}}(T) \ll \frac{T^{\alpha}}{(\log T)^{2}}$.

\section{Remarks:}

(1) The number of pairs of tangent circles in $\mathcal{P}$ of curvatures at most $T$ is equal to $3 N_{\mathcal{P}}(T)$ up to an additive constant (see Lemma 2.5). Therefore, in light of Theorem 1.1 the upper bounds in Theorem 1.4 are only off by a constant multiple from the expected asymptotics.

\footnotetext{
${ }^{1}$ Arguably the most elegant formulation of this theorem is the following excerpt from the poem "The Kiss Precise" by Nobel Laureate Sir Fredrick Soddy 44]: 
(2) A suitably modified version of Conjecture 1.4 in [4, a generalization of Schinzel's hypothesis, implies that for some $c, c_{2}>0$,

$$
\pi^{\mathcal{P}}(T) \sim c \cdot \frac{T^{\alpha}}{\log T} \quad \text { and } \quad \pi_{2}^{\mathcal{P}}(T) \sim c_{2} \cdot \frac{T^{\alpha}}{(\log T)^{2}}
$$

(see the discussion in [4, Ex D]). The constants $c$ and $c_{2}$ are detailed in [13].

Orbital counting of a Kleinian group in a cone: By Descartes' theorem (1.2), any Descartes quadruple $(a, b, c, d)$ lies on the cone $Q(x)=0$, where $Q$ denotes the quadratic form

$$
Q(a, b, c, d)=2\left(a^{2}+b^{2}+c^{2}+d^{2}\right)-(a+b+c+d)^{2} .
$$

In light of (1.3), one can "flip" the quadruple $(a, b, c, d)$ into $\left(a, b, c, d^{\prime}\right)$ via leftmultiplication by $S_{4}$, where

$$
\begin{array}{rlr}
S_{1}=\left(\begin{array}{cccc}
-1 & 2 & 2 & 2 \\
0 & 1 & 0 & 0 \\
0 & 0 & 1 & 0 \\
0 & 0 & 0 & 1
\end{array}\right), & S_{2}=\left(\begin{array}{cccc}
1 & 0 & 0 & 0 \\
2 & -1 & 2 & 2 \\
0 & 0 & 1 & 0 \\
0 & 0 & 0 & 1
\end{array}\right), \\
S_{3}=\left(\begin{array}{cccc}
1 & 0 & 0 & 0 \\
0 & 1 & 0 & 0 \\
2 & 2 & -1 & 2 \\
0 & 0 & 0 & 1
\end{array}\right), & S_{4}=\left(\begin{array}{cccc}
1 & 0 & 0 & 0 \\
0 & 1 & 0 & 0 \\
0 & 0 & 1 & 0 \\
2 & 2 & 2 & -1
\end{array}\right) .
\end{array}
$$

Let $\mathcal{A}$ denote the so-called Apollonian group generated by these reflections, that is, $\mathcal{A}=\left\langle S_{1}, S_{2}, S_{3}, S_{4}\right\rangle$, and let $\mathrm{O}_{Q}$ be the orthogonal group preserving $Q$. One can check that $\mathcal{A}<\mathrm{O}_{Q}(\mathbb{Z})$ and that $Q$ has signature $(3,1)$. Therefore $\mathcal{A}$ is a Kleinian group; moreover $\mathcal{A}$ is of infinite index in $\mathrm{O}_{Q}(\mathbb{Z})$.

For a fixed packing $\mathcal{P}$, there is a labeling by the Apollonian group $\mathcal{A}$ of all the (unordered) Descartes quadruples in $\mathcal{P}$. Moreover the counting problems for $N^{\mathcal{P}}(T)$ and $N_{2}^{\mathcal{P}}(T)$ for $\mathcal{P}$ bounded can be reduced to counting elements in the orbit $\xi_{\mathcal{P}} \cdot \mathcal{A}^{t} \subset \mathbb{R}^{4}$ of maximum norm at most $T$, where $\xi_{\mathcal{P}}$ is the unique root quadruple of $\mathcal{P}$ (see Definition 2.2 and Lemma 2.5). For $\mathcal{P}$ congruent to Figure 3 , the same reduction holds with $\xi_{P}$ given by $(0,0, c, c)$ where $c$ is the curvature of the largest circle in $\mathcal{P}$.

We prove the following more general counting theorem: Let $\iota: \mathrm{PSL}_{2}(\mathbb{C}) \rightarrow$ $\mathrm{SO}_{F}(\mathbb{R})$ be a real linear representation, where $F$ is a real quadratic form in 4 variables with signature $(3,1)$. Let $\Gamma<\mathrm{PSL}_{2}(\mathbb{C})$ be a geometrically finite Kleinian group. The limit set $\Lambda(\Gamma)$ of $\Gamma$ is the set of accumulation points of $\Gamma$-orbits in the ideal boundary $\partial_{\infty}\left(\mathbb{H}^{3}\right)$ of the hyperbolic space $\mathbb{H}^{3}$. We assume that the Hausdorff dimension $\delta_{\Gamma}$ of $\Lambda(\Gamma)$ is strictly greater than one.

Theorem 1.5. Let $v_{0} \in \mathbb{R}^{4}$ be a non-zero vector lying in the cone $F=0$ with a discrete orbit $v_{0} \Gamma \subset \mathbb{R}^{4}$. Then for any norm $\|\cdot\|$ on $\mathbb{R}^{4}$, as $T \rightarrow \infty$,

$$
\#\left\{v \in v_{0} \Gamma:\|v\|<T\right\} \sim c \cdot T^{\delta_{\Gamma}},
$$

where $c>0$ is explicitly given in Theorem 7.1 .

There are two main difficulties preventing existing counting methods from tackling the above asymptotic formula. The first is that $\Gamma$ is not required to be a lattice in $\mathrm{PSL}_{2}(\mathbb{C})$ (recall that the Apollonian group $\mathcal{A}$ has infinite index in $\mathrm{O}_{Q}(\mathbb{Z})$ ), so Patterson-Sullivan theory enters in the spectral decomposition of the hyperbolic 
manifold $\Gamma \backslash \mathbb{H}^{3}$. The second difficulty noted by Sarnak in [40] stems from the fact that the stabilizer of $v_{0}$ in $\Gamma$ may not have enough unipotent elements; in the application to Apollonian packings, the stabilizer is indeed either finite or a rank one abelian subgroup, whereas the stabilizer of $v_{0}$ in the ambient group $G$ is a compact extension of a rank two abelian subgroup.

In the similar situation of an infinite area hyperbolic surface, that is, when $\Gamma<\mathrm{PSL}_{2}(\mathbb{R})$, the counting problem in a cone with respect to a Euclidean norm was solved in Kontorovich's thesis 21, under the assumption that the stabilizer of $v_{0}$ in $\Gamma$ is co-compact in the stabilizer of $v_{0}$ in $\mathrm{PSL}_{2}(\mathbb{R})$. The methods developed here are quite different. Our approach to the counting problem is via the equidistribution of expanding closed horospheres on hyperbolic 3-manifolds (see the next subsection for a more detailed discussion). Our proof of the equidistribution works for hyperbolic surfaces as well, and in particular solves the counting problem in 21] for any norm and without assumptions on the stabilizer. In 22, we apply the methods of this paper to the problem of thin orbits of Pythagorean triples having few prime factors.

Equidistribution of expanding horospheres in hyperbolic 3-manifolds: Let $\Gamma$ be a geometrically finite torsion-free discrete subgroup of $\mathrm{PSL}_{2}(\mathbb{C})$. The main ingredient of our proof of Theorem [1.5] is the equidistribution of expanding closed horospheres in $\Gamma \backslash \mathbb{H}^{3}$.

The group $G=\mathrm{PSL}_{2}(\mathbb{C})$ is the group of orientation-preserving isometries of the hyperbolic space $\mathbb{H}^{3}=\left\{\left(x_{1}, x_{2}, y\right): y>0\right\}$. The invariant measure on $\mathbb{H}^{3}$ for the action of $G$ and the Laplace operator are given respectively by

$$
\frac{d x_{1} d x_{2} d y}{y^{3}} \text { and } \Delta=-y^{2}\left(\frac{\partial^{2}}{\partial y^{2}}+\frac{\partial^{2}}{\partial x_{1}^{2}}+\frac{\partial^{2}}{\partial x_{2}^{2}}\right)+y \frac{\partial}{\partial y} \text {. }
$$

Set

$$
\begin{aligned}
N & =\left\{n_{x}:=\left(\begin{array}{ll}
1 & x \\
0 & 1
\end{array}\right): x \in \mathbb{C}\right\} \\
A & =\left\{a_{y}:=\left(\begin{array}{cc}
\sqrt{y} & 0 \\
0 & \sqrt{y}^{-1}
\end{array}\right): y>0\right\} \\
K & =\left\{g \in G: \bar{g}^{t} g=I\right\} \text { and } \\
M & =\left\{\left(\begin{array}{cc}
e^{i \theta} & 0 \\
0 & e^{-i \theta}
\end{array}\right): \theta \in \mathbb{R}\right\}
\end{aligned}
$$

By the Iwasawa decomposition $G=N A K$, any element $g \in G$ can be written uniquely as $g=n_{x} a_{y} k$ with $n_{x} \in N, a_{y} \in A$, and $k \in K$. Via the map

$$
n_{x} a_{y}(0,0,1)=(x, y),
$$

the hyperbolic space $\mathbb{H}^{3}$ and its unit tangent bundle $\mathrm{T}^{1}\left(\mathbb{H}^{3}\right)$ can be identified with the quotients $G / K$ and $G / M$, respectively.

Denoting by $[u]$ the image of $u \in G$ under the quotient map $G \rightarrow G / M$, the horospheres correspond to $N$-leaves $[u] N=[u N]$ in $G / M$; note that this is well defined as $M$ normalizes $N$. For a closed horosphere $\Gamma \backslash \Gamma[u] N$, the translates $\Gamma \backslash \Gamma[u] N a_{y}$ represent closed horospheres $\Gamma \backslash \Gamma\left[u a_{y}\right] N$, which are expanding as $y \rightarrow 0$.

In the case of a hyperbolic surface $\Gamma \backslash \mathbb{H}^{2}$ of finite area, it is a theorem of Sarnak's 39] that such long horocycle flows are equidistributed with respect to the 


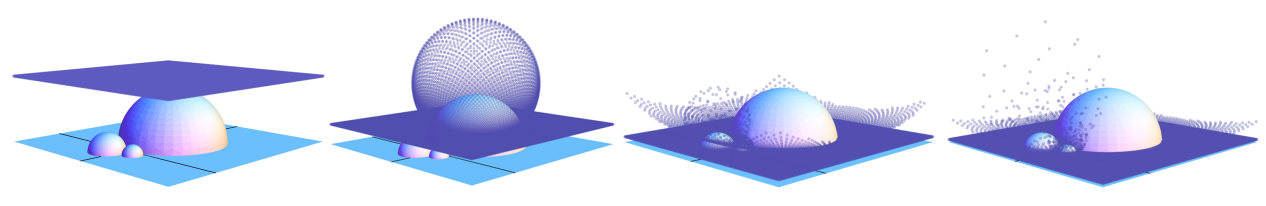

FiguRE 5. Equidistribution of expanding horospheres

Haar measure. The equidistribution of expanding horospheres for any finite volume hyperbolic manifold can also be proved using the mixing of geodesic flows. This approach appears already in Margulis's 1970 thesis [25]; see also [12].

In what follows we describe our equidistribution result for expanding closed horospheres on any geometrically finite hyperbolic 3-manifold.

Assuming $\delta_{\Gamma}>1$, Sullivan [45] showed, generalizing the work of Patterson 34, that there exists a unique positive eigenfunction $\phi_{0}$ of the Laplacian operator $\Delta$ on $\Gamma \backslash \mathbb{H}^{3}$ of lowest eigenvalue $\delta_{\Gamma}\left(2-\delta_{\Gamma}\right)$ and of unit $L^{2}$-norm, that is, $\int_{\Gamma \backslash \mathbb{H}^{3}} \phi_{0}\left(x_{1}, x_{2}, y\right)^{2} \frac{1}{y^{3}} d x_{1} d x_{2} d y=1$. Moreover the base eigenvalue-value $\delta_{\Gamma}\left(2-\delta_{\Gamma}\right)$ is isolated in the $L^{2}$-spectrum of $\Delta$ by Lax and Phillips [24.

Note that the closed leaf $\Gamma \backslash \Gamma N$ inside $\mathrm{T}^{1}\left(\Gamma \backslash \mathbb{H}^{3}\right)$ is an embedding of one of the following: a complex plane, a cylinder, or a torus. As $\phi_{0}>0$, it is a priori not clear whether the integral

$$
\phi_{0}^{N}\left(a_{y}\right):=\int_{n_{x} \in(N \cap \Gamma) \backslash N} \phi_{0}(x, y) d x
$$

converges. We show that for any $y>0$, the integral $\phi_{0}^{N}\left(a_{y}\right)$ does converge and is of the form

$$
\phi_{0}^{N}\left(a_{y}\right)=c_{\phi_{0}} y^{2-\delta_{\Gamma}}+d_{\phi_{0}} y^{\delta_{\Gamma}}
$$

for some constants $c_{\phi_{0}}>0$ and $d_{\phi_{0}} \geq 0$. By $f(y) \sim g(y)$ with $y \rightarrow 0$, we mean that $\lim _{y \rightarrow 0} \frac{f(y)}{g(y)}=1$. The measure $d n$ on $N$ is Lebesgue: $d n_{x}=d x_{1} d x_{2}$.

Theorem 1.7. Let $\Gamma<G$ be a geometrically finite torsion-free discrete subgroup with $\delta_{\Gamma}>1$ and let $\Gamma \backslash \Gamma N$ be closed. There exists $\epsilon>0$ such that for any $\psi \in$ $C_{c}^{\infty}(\Gamma \backslash G)^{K}=C_{c}^{\infty}\left(\Gamma \backslash \mathbb{H}^{3}\right)$, and for all small $y>0$,

$$
\int_{(N \cap \Gamma) \backslash N} \psi\left(\Gamma \backslash \Gamma n a_{y}\right) d n=c_{\phi_{0}} \cdot\left\langle\psi, \phi_{0}\right\rangle_{L^{2}\left(\Gamma \backslash \mathbb{H}^{3}\right)} \cdot y^{2-\delta_{\Gamma}}\left(1+O\left(y^{\epsilon}\right)\right),
$$

where the implied constant depends only on the Sobolev norm of $\psi$.

Thus as $y \rightarrow 0$, the integral of any function $\psi \in C_{c}(\Gamma \backslash G)^{K}$ along the orthogonal translate $\Gamma \backslash \Gamma N a_{y}$ converges to 0 with the speed of order $y^{2-\delta_{\Gamma}}$. It also follows that for $\psi \in C_{c}(\Gamma \backslash G)^{K}$, as $y \rightarrow 0$,

$$
\int_{(N \cap \Gamma) \backslash N} \psi\left(\Gamma \backslash \Gamma n a_{y}\right) d n \sim\left\langle\psi, \phi_{0}\right\rangle \cdot \int_{(N \cap \Gamma) \backslash N} \phi_{0}\left(\Gamma \backslash \Gamma n a_{y}\right) d n .
$$

We denote by $\tilde{\Omega}_{\Gamma}$ the set of vectors $(p, \vec{v})$ in the unit tangent bundle $\mathrm{T}^{1}\left(\mathbb{H}^{3}\right)$ such that the end point of the geodesic ray tangent to $\vec{v}$ belongs to the limit set $\Lambda(\Gamma)$ and by $\hat{\Omega}_{\Gamma}$ its image under the projection of $\mathrm{T}^{1}\left(\mathbb{H}^{3}\right)$ to $\mathrm{T}^{1}\left(\Gamma \backslash \mathbb{H}^{3}\right)$.

Roblin [38, generalizing the work of Burger [7, showed that, up to a constant multiple, there exists a unique Radon measure $\hat{\mu}$ on $T^{1}\left(\Gamma \backslash \mathbb{H}^{3}\right)$ invariant for the 
horospherical foliations which is supported on $\hat{\Omega}_{\Gamma}$ and gives zero measure to all closed horospheres.

In the appendix, written jointly by Shah and the second-named author, the following theorem is deduced from Theorem 1.7 based on the aforementioned measure classification of Burger and Roblin. In view of the isomorphism $\mathrm{T}^{1}\left(\Gamma \backslash \mathbb{H}^{3}\right)=$ $\Gamma \backslash G / M$, the following theorem shows that the orthogonal translations of closed horospheres in the expanding direction are equidistributed in $\mathrm{T}^{1}\left(\Gamma \backslash \mathbb{H}^{3}\right)$ with respect to the Burger-Roblin measure $\hat{\mu}$.

Theorem 1.8. For any $\psi \in C_{c}(\Gamma \backslash G)^{M}$, as $y \rightarrow 0$,

$$
\int_{(N \cap \Gamma) \backslash N} \psi\left(\Gamma \backslash \Gamma n a_{y}\right) d n \sim c_{\phi_{0}} \cdot y^{2-\delta_{\Gamma}} \cdot \hat{\mu}(\psi),
$$

where $\hat{\mu}$ is normalized so that $\hat{\mu}\left(\phi_{0}\right)=1$.

Theorem 1.8 was proved by Roblin 38 when $(N \cap \Gamma) \backslash N$ is compact with a different interpretation of the constant $c_{\phi_{0}}$. His proof does not yield an effective version as in Theorem 1.7 but works for any $\delta_{\Gamma}>0$.

We also remark that the quotient type equidistribution results for non-closed horocycles for geometrically finite surfaces were established by Schapira [1].

We conclude the introduction by giving a brief outline of the proof of Theorem 1.7. By Dal'bo (Theorem 3.3), we have the following classification of closed horospheres in terms of its base point in the boundary $\partial_{\infty}\left(\mathbb{H}^{3}\right): \Gamma \backslash \Gamma N$ is closed if and only if either $\infty \notin \Lambda(\Gamma)$ or $\infty$ is a bounded parabolic fixed point (see Definition 3.1). This classification is used repeatedly in our analysis, establishing the following facts:

(1) For any bounded subset $B \subset(N \cap \Gamma) \backslash N$ which properly covers $(N \cap$ $\Gamma) \backslash(\Lambda(\Gamma)-\{\infty\})$ (cf. Definition 4.5),

$$
\int_{(N \cap \Gamma) \backslash N} \phi_{0}\left(n a_{y}\right) d n=\int_{B} \phi_{0}\left(n a_{y}\right) d n+O\left(y^{\delta_{\Gamma}}\right) .
$$

(2) Denoting by $\rho_{B, \epsilon} \in C_{c}^{\infty}(\Gamma \backslash G)$ the $\epsilon$-approximation of $B$ in the transversal direction,

$$
\int_{B} \phi_{0}\left(n a_{y}\right) d n=\left\langle a_{y} \phi_{0}, \rho_{B, \epsilon}\right\rangle+O\left(\epsilon y^{2-\delta_{\Gamma}}\right)+O\left(y^{\delta_{\Gamma}}\right) .
$$

(3) For $\psi \in C_{c}\left(\Gamma \backslash \mathbb{H}^{3}\right)$, there exists a compact subset $B=B(\operatorname{supp}(\psi)) \subset$ $(N \cap \Gamma) \backslash N$ such that for all $0<y<1$,

$$
\int_{(N \cap \Gamma) \backslash N} \psi\left(n a_{y}\right) d n=\int_{B} \psi\left(n a_{y}\right) d n .
$$

These facts allow us to focus on the integral of $\psi$ over a compact region, say, $B$, of $(N \cap \Gamma) \backslash N$ instead of the whole space. In approximating $\int_{B} \psi\left(n a_{y}\right) d n$ with $\left\langle a_{y} \psi, \rho_{B, \epsilon}\right\rangle$, the usual argument based on the contracting property along the stable horospheres is not sufficient: the error terms combine with those coming from the spectral gap to overtake the main term! We develop a recursive argument, which improves the error upon each iteration, and halts in finite time, once the main term is dominant. Using the spectral theory of $L^{2}(\Gamma \backslash G)$ along with the assumption $\delta_{\Gamma}>1$, we get a control of the main term of $\left\langle a_{y} \psi, \rho_{B, \epsilon}\right\rangle$ as $\left\langle\psi, \phi_{0}\right\rangle \cdot\left\langle a_{y} \phi_{0}, \rho_{B, \epsilon}\right\rangle$. 
For the application to Apollonian packings, we need to consider the max norm, which necessitates the extension of our argument to the unit tangent bundle, that is, the deduction of Theorem 1.8 from 1.7 as done in the appendix.

We finally remark that the power savings error term in (1) of Theorem 1.7 is crucial to proving Theorem 1.4.

After this paper was submitted, the asymptotic formula for counting circles in a curvilinear triangle of any Apollonian packing was obtained in [33. See also [32] for similar counting results for hyperbolic and spherical Apollonian circle packings. We also refer to 31] for a survey on recent progress on counting circles.

\section{Reduction to orbital COUNTing}

2.1. Apollonian group. In a quadruple of mutually tangent circles, the curvatures $a, b, c, d$ satisfy the Descartes equation:

$$
2\left(a^{2}+b^{2}+c^{2}+d^{2}\right)=(a+b+c+d)^{2}
$$

as observed by Descartes in 1643 (see 9 for a proof).

Any quadruple $(a, b, c, d)$ satisfying this equation is called a Descartes quadruple. A set of four mutually tangent circles with disjoint interiors is called a Descartes configuration.

We denote by $Q$ the Descartes quadratic form given by

$$
Q(a, b, c, d)=a^{2}+b^{2}+c^{2}+d^{2}-\frac{1}{2}(a+b+c+d)^{2} .
$$

Hence $v=(a, b, c, d)$ is a Descartes quadruple if and only if $Q(v)=0$. The orthogonal group corresponding to $Q$ is given by

$$
\mathrm{O}_{Q}=\left\{g \in \mathrm{GL}_{4}: Q\left(v g^{t}\right)=Q(v) \text { for all } v \in \mathbb{R}^{4}\right\} .
$$

One can easily check that the Apollonian group $\mathcal{A}:=\left\langle S_{1}, S_{2}, S_{3}, S_{4}\right\rangle$ defined in the introduction is a subgroup of $\mathrm{O}_{Q}(\mathbb{Z}):=\mathrm{O}_{Q} \cap \mathrm{GL}_{4}(\mathbb{Z})$.

Definition 2.1. (1) For $\mathcal{P}$ bounded, denote by $N^{\mathcal{P}}(T)$ the number of circles in $\mathcal{P}$ in the packing whose curvature is at most $T$, i.e., whose radius is at least $1 / T$. Denote by $N_{2}^{\mathcal{P}}(T)$ the number of pairs of tangent circles in $\mathcal{P}$ of curvatures at most $T$.

(2) For $\mathcal{P}$ congruent to the packing in Figure 3, $N^{\mathcal{P}}(T)$ denotes the number of circles between two largest tangent circles including the lines and the largest circles. Similarly, $N_{2}^{\mathcal{P}}(T)$ denotes the number of unordered pairs of tangent circles between two largest tangent circles including the pairs containing the lines and the largest circles.

(3) For $\mathcal{P}$ bounded, denote by $\pi^{\mathcal{P}}(T)$ the number of prime circles in $\mathcal{P}$ of curvature at most $T$. Denote by $\pi_{2}^{\mathcal{P}}(T)$ the number of twin prime circles in $\mathcal{P}$ of curvatures at most $T$.

(4) For $\mathcal{P}$ congruent to the packing in Figure 3, one alters the definition of $\pi^{\mathcal{P}}(T)$ and $\pi_{2}^{\mathcal{P}}(T)$ to count prime circles in a fixed period.

We will interpret $N^{\mathcal{P}}(T)$ and $N_{2}^{\mathcal{P}}(T)$ as orbital counting functions on $\xi \mathcal{A}^{t}$ for a carefully chosen Descartes quadruple $\xi$ of $\mathcal{P}$.

Definition 2.2. A Descartes quadruple $v=(a, b, c, d)$ with $a+b+c+d>0$ is a root quadruple if $a \leq 0 \leq b \leq c \leq d$ and $a+b+c \geq d$. 
If $\mathcal{P}$ is bounded, Theorem 3.2 in [15] shows that $\mathcal{P}$ contains a unique Descartes root quadruple $\xi:=(a, b, c, d)$ with $a<0$.

Theorem 2.3 ([15, Thm. 3.3]). The set of curvatures occurring in $\mathcal{P}$, counted with multiplicity, consists of the four entries in $\xi$, together with the largest entry in each vector $\xi \gamma^{t}$ as $\gamma$ runs over all non-identity elements of the Apollonian group $\mathcal{A}$.

In [15, this is stated only for an integral Apollonian packing, but the same proof works for any bounded packing. Let $w^{(n)}$ be a non-returning walk away from the root quadruple $\xi$ along the Apollonian group, i.e., $w^{(n)}=\xi S_{i_{1}}^{t} \cdots S_{i_{n}}^{t}$ with $S_{i_{k}} \neq S_{i_{k+1}}, 1 \leq k \leq n-1$. Then the key observation in the proof of the above theorem is that $w^{(n)}$ is obtained from $w^{(n-1)}$ by changing one entry, and moreover the new entry inserted is always the largest entry in the new vector.

This theorem yields that for $T \gg 1$,

$$
N^{\mathcal{P}}(T)=\#\left\{\gamma \in \mathcal{A}:\left\|\xi \gamma^{t}\right\|_{\max }<T\right\}+3 .
$$

Consider the repeated generations of $\mathcal{P}$ with 4 initial circles given by the root quadruple. Then a geometric version of Theorem 2.3 is that for $n \geq 1$, each reduced word $\gamma=S_{i_{n}} \cdots S_{i_{1}}$ of length $n$ corresponds to exactly one new circle, say $C_{\gamma}$, added at the $n$-th generation, and the curvature of $C_{\gamma}$ is the maximum among the entries of the quadruple $\xi \gamma^{t}$ (cf. [16, Section 4]). Thus the correspondence $\phi: \gamma \mapsto C_{\gamma}$ establishes a bijection between the set of all non-identity elements of $\mathcal{A}$ and the set of all circles not in the zeroth generation. Hence the set $\left\{C_{\gamma} \mid \gamma \neq e,\left\|\xi \gamma^{t}\right\|<T\right\}$ gives all circles (excluding those 4 initial circles) of curvature at most $T$.

For each $\gamma \neq e$ in $\mathcal{A}$, set

$$
\phi_{2}(\gamma)=\left\{\left\{C_{\gamma}, C_{\gamma}(1)\right\},\left\{C_{\gamma}, C_{\gamma}(2)\right\},\left\{C_{\gamma}, C_{\gamma}(3)\right\}\right\},
$$

where $C_{\gamma}(i), i=1,2,3$, are the three circles corresponding to the quadruple $\xi \gamma^{t}$ besides $C_{\gamma}$. Noting that each $\left(C_{\gamma}, C_{\gamma}(i)\right)$ gives a pair of tangent circles, we claim that every pair of tangent circles arises as one of the triples in the image of $\phi_{2}$, provided one of the circles in the pair does not come from the zeroth stage. If $C$ and $D$ form such a pair and are not from the initial stage, then they are different generations and it is obvious that no two circles in the same generation touch each other. If, say, $D$ is generated earlier than $C$, then for the element $\gamma \in \mathcal{A}$ giving $C=C_{\gamma}$, which is necessarily a non-identity element, $D$ must be one of the $C_{\gamma}(i)$ 's. This is because it is clear from the construction of the packing that every circle is tangent to only three circles from previous generations.

Therefore $\phi_{2}$ yields a one-to-three correspondence from $\mathcal{A} \backslash\{e\}$ to the set of all unordered pairs of tangent circles in $\mathcal{P}$, at least one circle of whose pair does not correspond to the root quadruple. Since there are 6 pairs arising from the 4 initial circles, we deduce that for $T \gg 1$,

$$
N_{2}^{\mathcal{P}}(T)=3 \cdot \#\left\{\gamma \in \mathcal{A}:\left\|\xi \gamma^{t}\right\|_{\max }<T\right\}+3 .
$$

The above argument establishing (2.4) was kindly explained to us by Peter Sarnak.

If $\mathcal{P}$ lies between two parallel lines, that is, congruent to Figure 3, there exists a unique $c>0$ such that $\mathcal{P}$ contains a Descartes quadruple $\xi:=(0,0, c, c)$.

In this case, the stabilizer of $\xi$ in $\mathcal{A}^{t}$ is generated by two reflections $S_{3}^{t}$ and $S_{4}^{t}$. One can directly verify that for all $T \gg 1$,

$$
N^{\mathcal{P}}(T)=\#\left\{v \in \xi \mathcal{A}^{t}:\|v\|_{\max }<T\right\}+3
$$


and

$$
N_{2}^{\mathcal{P}}(T)=3 \cdot \#\left\{v \in \xi \mathcal{A}^{t}:\|v\|_{\max }<T\right\}+3 .
$$

Lemma 2.5. Let $\mathcal{P}$ be either bounded or congruent to Figure 3.

(1) For all $T \gg 1$,

$$
N^{\mathcal{P}}(T)= \begin{cases}\# \operatorname{Stab}_{\mathcal{A}^{t}}(\xi) \cdot \#\left\{v \in \xi \mathcal{A}^{t}:\|v\|_{\max }<T\right\}+3 & \text { for } \mathcal{P} \text { bounded, } \\ \#\left\{v \in \xi \mathcal{A}^{t}:\|v\|_{\max }<T\right\}+3 & \text { otherwise. }\end{cases}
$$

(2) For all $T \gg 1$,

$$
N_{2}^{\mathcal{P}}(T)=3 N^{\mathcal{P}}(T)-6 .
$$

(3) The orbit $\xi \mathrm{A}^{t}$ is discrete in $\mathbb{R}^{4}$.

(4) For all $T \gg 1$,

$$
\pi^{\mathcal{P}}(T) \ll \sum_{i=1}^{4} \#\left\{v=\left(v_{1}, v_{2}, v_{3}, v_{4}\right) \in \xi \mathcal{A}^{t}:\|v\|_{\max }<T, v_{i} \text { is prime }\right\} .
$$

(5) For all $T \gg 1$,

$$
\pi_{2}^{\mathcal{P}}(T) \ll \sum_{1 \leq i \neq j \leq 4} \#\left\{v \in \xi \mathcal{A}^{t}:\|v\|_{\max }<T, v_{i}, v_{j} \text { are primes }\right\} .
$$

Proof. The first two claims follow immediately from the discussion above, noting that the stabilizer of $\xi$ in $\mathcal{A}^{t}$ is finite for $\mathcal{P}$ bounded. The third claim follows from the fact that $N^{\mathcal{P}}(T)<\infty$ for any $T>0$. For claims (4) and (5), note that for $\mathcal{P}$ bounded,

$$
\begin{aligned}
\pi^{\mathcal{P}}(T) & \leq 3+\#\left\{\gamma \in \mathcal{A}:\left\|\xi \gamma^{t}\right\|_{\max } \text { is prime }<T\right\} \\
& \ll \sum_{i=1}^{4} \#\left\{v=\left(v_{1}, v_{2}, v_{3}, v_{4}\right) \in \xi \mathcal{A}^{t}:\|v\|_{\max }<T, v_{i} \text { is prime }\right\}
\end{aligned}
$$

and

$$
\begin{aligned}
\pi_{2}^{\mathcal{P}}(T) & \leq 5+\#\left\{\gamma \in \mathcal{A}:\left\|\xi \gamma^{t}\right\|_{\max } \text { is prime }<T, \text { one more entry of } \xi \gamma^{t} \text { is prime }\right\} \\
& \ll \sum_{i=1}^{4} \sum_{j \neq i} \#\left\{v=\left(v_{1}, v_{2}, v_{3}, v_{4}\right) \in \xi \mathcal{A}^{t}:\|v\|_{\max }<T, v_{i}, v_{j} \text { are primes }\right\}
\end{aligned}
$$

The claims (4) and (5) for $\mathcal{P}$ congruent to Figure 3 can be shown similarly.

We remark that there are bounded packings which are not multiples of integral packings: for instance, $\xi=(3-2 \sqrt{3}, 1,1,1)$ is a Descartes root quadruple which defines a bounded Apollonian packing. This is obvious from the viewpoint of geometry, but it is not at all clear a priori that the orbit $\xi \mathcal{A}^{t}$ should be discrete. Note also that there are other unbounded packings: by applying a suitably chosen Möbius transformation to a given packing, one can arrive at a packing which spreads uncontrollably to the entire plane, or one which is fenced off along one side by a single line. 
2.2. The residual set. We consider the upper half-space model for the hyperbolic space:

$$
\mathbb{H}^{3}=\left\{\left(x_{1}, x_{2}, y\right) \in \mathbb{R}^{3}: y>0\right\}
$$

with metric given by $\frac{d x_{1}^{2}+d x_{2}^{2}+d y^{2}}{y^{3}}$. A discrete subgroup of $\operatorname{Isom}\left(\mathbb{H}^{3}\right)$ is called a Kleinian group.

The ideal boundary $\partial_{\infty}\left(\mathbb{H}^{3}\right)$ of $\mathbb{H}^{3}$ can be identified with the set of geodesic rays emanating from a fixed point $x_{0} \in \mathbb{H}^{3}$. The topology on $\partial_{\infty}\left(\mathbb{H}^{3}\right)$ is defined via the angles between corresponding rays: two geodesic rays are close if and only if the angle between the corresponding rays is small.

In the upper half-space model, we can identify $\partial_{\infty}\left(\mathbb{H}^{3}\right)$ with the extended complex plane $\left\{\left(x_{1}, x_{2}, 0\right)\right\} \cup\{\infty\}=\mathbb{C} \cup\{\infty\}$, which is homeomorphic to the sphere $\mathbb{S}^{2}$. The space $\mathbb{H}^{3}$ has the natural compactification $\mathbb{H}^{3} \cup \partial_{\infty}\left(\mathbb{H}^{3}\right)$ (cf. [20, 3.2]).

Definition 2.6. For a Kleinian group $\Gamma$, the limit set $\Lambda(\Gamma)$ of $\Gamma$ consists of limit points of an orbit $\Gamma z, z \in \mathbb{H}^{3}$, in the ideal boundary $\hat{\mathbb{C}}=\mathbb{C} \cup\{\infty\}$. We denote by $\delta_{\Gamma}$ the Hausdorff dimension of $\Lambda(\Gamma)$.

By Sullivan, $\delta_{\Gamma}$ is equal to the critical exponent of $\Gamma$ for geometrically finite $\Gamma$. In this subsection, we realize the action of $\mathcal{A}$ on Descartes quadruples arising from $\mathcal{P}$ as the action of a subgroup, $G_{\mathcal{A}}(\mathcal{P})$, of the Möbius transformations on $\widehat{\mathbb{C}}$ in a way that the residual set of $\mathcal{P}$ coincides with the limit set of $G_{\mathcal{A}}(\mathcal{P})$. The residual set $\Lambda(\mathcal{P})$ is defined to be the closure of all the circles in $\mathcal{P}$, or equivalently, the complement in $\hat{\mathbb{C}}$ of the interiors of all circles in the packing $\mathcal{P}$ (where the circles are oriented so that the interiors are disjoint).

An oriented Descartes configuration is a Descartes configuration in which the orientations of the circles are compatible in the sense that either the interiors of all four oriented circles are disjoint or the interiors are disjoint when all the orientations are reversed. Given an ordered configuration $\mathcal{D}$ of four oriented circles $\left(C_{1}, C_{2}, C_{3}, C_{4}\right)$ with curvatures $\left(b_{1}, b_{2}, b_{3}, b_{4}\right)$ and centers $\left\{\left(x_{i}, y_{i}\right): 1 \leq i \leq 4\right\}$, set

$$
W_{\mathcal{D}}:=\left(\begin{array}{llll}
\bar{b}_{1} & b_{1} & b_{1} x_{1} & b_{1} y_{1} \\
\bar{b}_{2} & b_{2} & b_{2} x_{2} & b_{2} y_{2} \\
\bar{b}_{3} & b_{3} & b_{3} x_{3} & b_{3} y_{3} \\
\bar{b}_{4} & b_{4} & b_{4} x_{4} & b_{4} y_{4}
\end{array}\right),
$$

where $\bar{b}_{i}$ is the curvature of the circle which is the reflection of $C_{i}$ through the unit circle centered at the origin, i.e., $\bar{b}_{i}=b_{i}\left(x_{i}^{2}+y_{i}^{2}\right)-b_{i}^{-1}$ if $b_{i} \neq 0$. If one of the circles, say $C_{i}$, is a line, we interpret the center $\left(x_{i}, y_{i}\right)$ as the outward unit normal vector and set $b_{i}=\bar{b}_{i}=0$.

Then by [16, Thm. 3.2], for any ordered and oriented Descartes configuration $\mathcal{D}$, the map $g \mapsto W_{\mathcal{D}}^{-1} g W_{\mathcal{D}}$ gives an isomorphism

$$
\psi_{\mathcal{D}}: \mathrm{O}_{Q}(\mathbb{R}) \rightarrow \mathrm{O}_{Q_{W}}(\mathbb{R})
$$

where $Q_{W}$ is the Wilker quadratic form:

$$
Q_{W}=\left(\begin{array}{cccc}
0 & -4 & 0 & 0 \\
-4 & 0 & 0 & 0 \\
0 & 0 & 2 & 0 \\
0 & 0 & 0 & 2
\end{array}\right)
$$




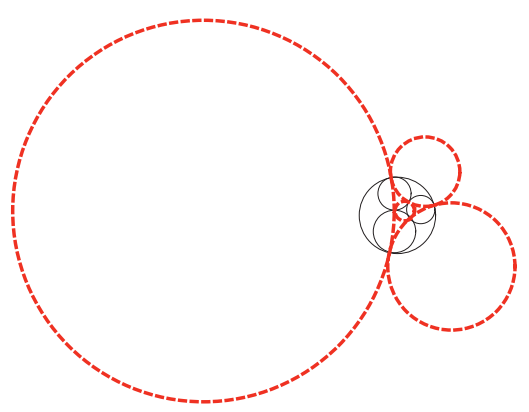

Figure 6. Action of $\mathcal{A}$ as Möbius transformations

On the other hand, if Möb(2) denotes the group of Möbius transformations and $\mathrm{GM}^{*}(2):=\operatorname{Möb}(2) \times\{ \pm I\}$ denotes the extended Möbius group, we have the following by Graham et al:

Theorem 2.7 ([16, Thm. 7.2]). There exists a unique isomorphism

$$
\pi: \mathrm{GM}^{*}(2) \rightarrow \mathrm{O}_{Q_{W}}(\mathbb{R})
$$

such that for any ordered and oriented Descartes configuration $\mathcal{D}$,

(1) $W_{\gamma(\mathcal{D})}=W_{\mathcal{D}} \pi(\gamma)^{-1} \quad$ for any $\gamma \in \operatorname{Möb}(2)$.

(2) $W_{-\mathcal{D}}=-W_{\mathcal{D}}$.

Let $\mathcal{D}_{0}=\left(C_{1}, C_{2}, C_{3}, C_{4}\right)$ denote the ordered and oriented Descartes configuration corresponding to the root quadruple $\xi$ of $\mathcal{P}$. We obtain the following isomorphism:

$$
\Phi_{\mathcal{D}_{0}}:=\pi^{-1} \circ \psi_{\mathcal{D}_{0}}: \mathrm{O}_{Q}(\mathbb{R}) \rightarrow \mathrm{GM}^{*}(2)
$$

Denote by $\mathfrak{s}_{i}:=\mathfrak{s}_{i}\left(\mathcal{D}_{0}\right)$ the Möbius transformation given by the inversion in the circle, say, $\hat{C}_{i}$, determined by the three intersection points of the circles $C_{j}$, $j \neq i$. Figure 6 depicts the root quadruple $\left(C_{1}, \ldots, C_{4}\right)$ as solid-lined circles and the corresponding dual quadruple $\left(\hat{C}_{1}, \ldots, \hat{C}_{4}\right)$ as dotted-lined circles.

Note that $\mathfrak{s}_{i}$ fixes $C_{j}, j \neq i$, and moves $C_{i}$ to the unique other circle that is tangent to the $C_{j}$ 's, $j \neq i$.

We set

$$
G_{\mathcal{A}}(\mathcal{P}):=\left\langle\mathfrak{s}_{1}, \mathfrak{s}_{2}, \mathfrak{s}_{3}, \mathfrak{s}_{4}\right\rangle .
$$

Lemma 2.8. For each $1 \leq i \leq 4$,

$$
\Phi_{\mathcal{D}_{0}}\left(S_{i}\right)=\mathfrak{s}_{i}
$$

hence

$$
\Phi_{\mathcal{D}_{0}}(\mathcal{A})=G_{\mathcal{A}}(\mathcal{P}) .
$$

Proof. If $\gamma_{i}:=\Phi_{\mathcal{D}_{0}}\left(S_{i}\right)$, then by (2.7),

$$
W_{\gamma_{i}\left(\mathcal{D}_{0}\right)}=W_{\mathcal{D}_{0}}\left(\psi_{\mathcal{D}_{0}} S_{i}\right)^{-1}=S_{i} W_{\mathcal{D}_{0}} .
$$

On the other hand, by [16, 3.25],

$$
W_{\mathfrak{s}_{i}\left(\mathcal{D}_{0}\right)}=S_{i} W_{\mathcal{D}_{0}} .
$$

Therefore

and hence $\gamma_{i}=\mathfrak{s}_{i}$.

$$
\gamma_{i}\left(\mathcal{D}_{0}\right)=\mathfrak{s}_{i}\left(\mathcal{D}_{0}\right)
$$




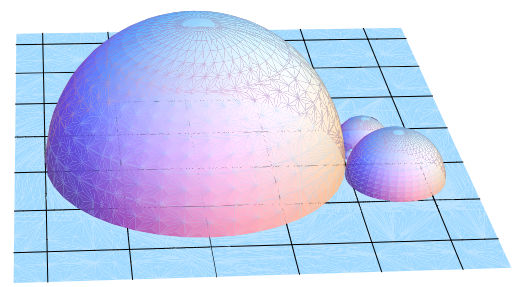

Figure 7. Fundamental domain

Each inversion $\mathfrak{s}_{i}$ extends uniquely to an isometry of the hyperbolic space $\mathbb{H}^{3}$, corresponding to the inversion with respect to the hemisphere whose boundary is $\hat{C}_{i}$. The intersection of the exteriors of these hemispheres is a fundamental domain for the action of $G_{\mathcal{A}}(\mathcal{P})$ on $\mathbb{H}^{3}$.

The Hausdorff dimension of $\Lambda(\mathcal{P})$, say $\alpha$, is independent of $\mathcal{P}$. Hirst showed in [18 that $\alpha$ is strictly between one and two. For our purpose, we only need to know that $\alpha>1$, though much more precise estimates were made by Boyd in [6] and McMullen 29].

Proposition 2.9. (1) $G_{\mathcal{A}}(\mathcal{P})$ is geometrically finite and discrete.

(2) We have $\Lambda\left(G_{\mathcal{A}}(\mathcal{P})\right)=\Lambda(\mathcal{P})$, and hence $\alpha=\delta_{G_{\mathcal{A}}(\mathcal{P})}$.

Proof. For simplicity set $\Gamma:=G_{\mathcal{A}}(\mathcal{P})$. The group $\Gamma$ is geometrically finite (that is, it admits a finite sided Dirichlet domain) by [20, Thm. 13.1] and discrete since $\mathcal{A}$ is discrete and $\Phi_{\mathcal{D}_{0}}$ is a topological isomorphism.

Clearly, $\Gamma$ is non-elementary. It is well known that $\Lambda(\Gamma)$ is the same as the set of all accumulation points in the orbit $x_{0}$ under $\Gamma$ for any (fixed) $x_{0} \in \hat{\mathbb{C}}$. On the other hand, by [16, Thm. 4.2], $\Lambda(\mathcal{P})$ is equal to the closure of all tangency points of circles in $\mathcal{P}$ and is invariant under $\Gamma$. This immediately yields

$$
\Lambda(\Gamma) \subset \Lambda(\mathcal{P}) .
$$

If $x_{0} \in \Lambda(\mathcal{P})$, then any neighborhood, say $U$, of $x_{0}$ contains infinitely many circles. Since $\bigcup_{1 \leq i \leq 4} \Gamma\left(C_{i}\right)$ is the set of all circles in $\mathcal{P}$, there exist $j$ and an infinite sequence $\gamma_{i} \in \Gamma$ such that $\gamma_{i}\left(C_{j}\right) \subset U$ for all $i$. Therefore $x_{0} \in \Lambda(\Gamma)$. This proves that $\Lambda(\mathcal{P}) \subset \Lambda(\Gamma)$.

Set $\Gamma_{\mathcal{P}}$ to be the subgroup of holomorphic elements, that is,

$$
\Gamma_{\mathcal{P}}:=G_{\mathcal{A}}(\mathcal{P}) \cap \mathrm{PSL}_{2}(\mathbb{C}),
$$

since $\operatorname{Möb}(2)$ is the semidirect product of complex conjugation with the subgroup $\mathrm{Möb}_{+}(2)=\mathrm{PSL}_{2}(\mathbb{C})$ of orientation-preserving transformations. Then the above lemma holds with $\Gamma_{\mathcal{P}}$ in place of $G_{\mathcal{A}}(\mathcal{P})$, as both properties are inherited by a subgroup of finite index. By the well-known Selberg's lemma, we can further replace $\Gamma_{\mathcal{P}}$ by a torsion-free subgroup of finite index.

2.3. Reduction to orbital counting for a Kleinian group. Let $G:=\mathrm{PSL}_{2}(\mathbb{C})$ and $\Gamma<G$ be a geometrically finite, torsion-free Kleinian subgroup. Suppose we are given a real linear representation $\iota: G \rightarrow \mathrm{SO}_{F}(\mathbb{R})$, where $F$ is a real quadratic form in 4 variables with signature $(3,1)$. As we prefer to work with a right action, we consider $\mathbb{R}^{4}$ as the set of row vectors and the action is given by $v g:=\iota(g) v^{t}$ for $g \in G$ and $v \in \mathbb{R}^{4}$. 
By Lemma 2.5 and the discussions in the previous subsection, Theorem 1.1 follows from:

Theorem 2.10. Suppose $\delta_{\Gamma}>1$. Let $v_{0} \in \mathbb{R}^{4}$ be a non-zero vector lying in the cone $F=0$ with a discrete orbit $v_{0} \Gamma \subset \mathbb{R}^{4}$. Then for any norm $\|\cdot\|$ on $\mathbb{R}^{4}$, there exists $c>0$ such that

$$
\#\left\{v \in v_{0} \Gamma:\|v\|<T\right\} \sim c \cdot T^{\delta_{\Gamma}} .
$$

It also follows from Lemma 2.5 that this theorem implies that

$$
N_{2}^{\mathcal{P}}(T) \sim(3 c) \cdot T^{\alpha}
$$

for the same $c$ as in Theorem 1.1.

\section{Geometry of Closed horospheres on $\mathrm{T}^{1}\left(\Gamma \backslash \mathbb{H}^{3}\right)$}

Let $G=\mathrm{PSL}_{2}(\mathbb{C})$ and $\mathbb{H}^{3}=\left\{\left(x_{1}, x_{2}, y\right): y>0\right\}$. We use the notation $N, A$, $K, M, n_{x}$ and $a_{y}$ as defined in (1.6). Throughout this section, we suppose that $\Gamma<G$ is a torsion-free, non-elementary (i.e., $\Lambda(\Gamma)$ consists of more than 2 points) and geometrically finite Kleinian group.

Definition 3.1. (1) A point $\xi \in \Lambda(\Gamma)$ is called a parabolic fixed point if $\xi$ is fixed by a parabolic isometry of $\Gamma$ (that is, an element of trace \pm 2 ). The rank of a parabolic fixed point $\xi$ is defined to be the rank of the abelian group $\Gamma_{\xi}$ which stabilizes $\xi$.

(2) A parabolic fixed point $\xi$ is called a bounded parabolic if it is of rank 2 or if there exists a pair of two open disjoint discs in $\hat{\mathbb{C}}$ whose union, say $U$, is precisely $\Gamma_{\xi}$-invariant (that is, $U$ is invariant by $\Gamma_{\xi}$ and $\gamma(U) \cap U \neq \emptyset$ for all $\gamma \in \Gamma$ not belonging to $\Gamma_{\xi}$ ). Or equivalently, $\xi$ is bounded parabolic if $\Gamma_{\xi} \backslash(\Lambda(\Gamma)-\{\xi\})$ is compact.

(3) A point $\xi \in \Lambda(\Gamma)$ is called a point of approximation if there exist a sequence $\gamma_{i} \in \Gamma$ and $z \in \mathbb{H}^{3}$ such that $\gamma_{i} z \rightarrow \xi$ and $\gamma_{i} z$ is within a bounded distance from the geodesic ray ending at $\xi$.

Since $\Gamma$ is geometrically finite, it is known by the work of Beardon and Maskit 2] that any limit point $\xi \in \Lambda(\Gamma)$ is either a point of approximation or a bounded parabolic fixed point.

We denote by Vis the visual map from $\mathrm{T}^{1}\left(\mathbb{H}^{3}\right)$ to the ideal boundary $\partial_{\infty}\left(\mathbb{H}^{3}\right)$ which maps the vector $(p, \vec{v})$ to the end of the geodesic ray tangent to $\vec{v}$.

Definition 3.2. - We denote by $\tilde{\Omega}_{\Gamma} \subset \mathrm{T}^{1}\left(\mathbb{H}^{3}\right)$ the set of vectors $(p, \vec{v})$ whose image under the visual map belongs to $\Lambda(\Gamma)$.

- We denote by $\hat{\Omega}_{\Gamma}$ the image of $\tilde{\Omega}_{\Gamma}$ under the projection $\mathrm{T}^{1}\left(\mathbb{H}^{3}\right) \rightarrow \mathrm{T}^{1}\left(\Gamma \backslash \mathbb{H}^{3}\right)$.

We note that $\tilde{\Omega}_{\Gamma}$ is a closed set consisting of horospheres. For comparison, if $\Gamma$ has finite co-volume, then $\Lambda_{\Gamma}=\mathbb{C} \cup\{\infty\}$ and $\hat{\Omega}_{\Gamma}=\mathrm{T}^{1}\left(\Gamma \backslash \mathbb{H}^{3}\right)$.

For $u \in \mathrm{PSL}_{2}(\mathbb{C})$, we denote by $[u]$ its image in $\mathrm{PSL}_{2}(\mathbb{C}) / M$, which we may consider as a vector in $\mathrm{T}^{1}\left(\mathbb{H}^{3}\right)$. The horosphere determined by the vector $[u]$ in $\Gamma \backslash \mathrm{T}^{1}\left(\mathbb{H}^{3}\right)$ corresponds to $\Gamma \backslash \Gamma u N M / M$, which will be simply denoted by $\Gamma \backslash \Gamma[u] N$. We note that $u(\infty)$ is identified with $\operatorname{Vis}[u]$. 
Theorem 3.3 (Dal'bo, [10]). Let $u \in \mathrm{PSL}_{2}(\mathbb{C})$.

(1) If $u(\infty) \in \Lambda(\Gamma)$ is a point of approximation, then $\Gamma \backslash \Gamma[u] N$ is dense in $\hat{\Omega}_{\Gamma}$.

(2) The orbit $\Gamma \backslash \Gamma u N$ is closed in $\Gamma \backslash G$ if and only if either $u(\infty) \notin \Lambda(\Gamma)$ or $u(\infty)$ is a bounded parabolic fixed point for $\Gamma$.

The first claim was proved in [10, Prop. $\mathrm{C}$ and Cor. 1] under the condition that the length spectrum of $\Gamma$ is not discrete in $\mathbb{R}$. As remarked there, this condition holds for non-elementary Kleinian groups by 17. The second claim follows from [10, Prop. C and Cor. 1] together with the following lemma.

Lemma 3.4. (1) We have $\Gamma \cap N M=\Gamma \cap N$.

(2) The orbit $\Gamma \backslash \Gamma N M$ is closed if and only if $\Gamma \backslash \Gamma N$ is closed.

Proof. If $\gamma \in \Gamma \cap N M$, but not in $N$, then $\operatorname{Tr}^{2}(\gamma)$ is real and $0 \leq \operatorname{Tr}^{2}(\gamma)<4$, where $\operatorname{Tr}(\gamma)$ denotes the trace of $\gamma$. Hence $\gamma$ is an elliptic element. As $\Gamma$ is discrete, $\gamma$ must be of finite order. However $\Gamma$ is torsion-free, which forces $\gamma=e$. This proves the first claim. The second claim follows easily since the first claim implies that the inclusion map $\Gamma \cap N \backslash N \rightarrow \Gamma \cap N M \backslash N M$ is proper.

It follows that if $\Gamma \backslash \Gamma N$ is closed in $\Gamma \backslash \mathrm{PSL}_{2}(\mathbb{C})$, then $\Gamma \backslash \Gamma N$ is either

(1) the embedding of a plane if $\infty \notin \Lambda(\Gamma)$;

(2) the embedding of a cylinder if $\infty$ is a bounded parabolic fixed point of rank one; or

(3) the embedding of a torus if $\infty$ is a bounded parabolic fixed point of rank two.

For $X \subset \mathbb{H}^{3} \cup \partial_{\infty}\left(\mathbb{H}^{3}\right), \bar{X}$ denotes its closure in $\mathbb{H}^{3} \cup \partial_{\infty}\left(\mathbb{H}^{3}\right)$.

Proposition 3.5. Assume that $\Gamma \backslash \Gamma N$ is closed. There exists a finite-sided fundamental polyhedron $\mathcal{F} \subset \mathbb{H}^{3}$ for the action of $\Gamma$, and also a fundamental domain $\mathcal{F}_{N} \subset \mathbb{C}$ for the action of $N \cap \Gamma$ such that for some $r \gg 1$ and for some finite subset $I_{\Gamma} \subset \Gamma$,

$$
\left\{\left(x_{1}, x_{2}, y\right) \in \mathbb{H}^{3}: x_{1}+i x_{2} \in \mathcal{F}_{N}, x_{1}^{2}+x_{2}^{2}+y^{2}>r\right\} \subset \bigcup_{\gamma \in I_{\Gamma}} \gamma \mathcal{F} .
$$

Proof. Choose a finite-sided fundamental polyhedron $\mathcal{F}$ for $\Gamma$ with $\infty \in \overline{\mathcal{F}}$. If $\infty \notin \Lambda(\Gamma)$, then $\infty$ lies in the interior of $\bigcup_{\gamma \in I_{\Gamma}} \gamma \mathcal{F}$ for some finite $I_{\Gamma} \subset \Gamma$. As the exteriors of hemispheres form a basis of neighborhoods of $\infty$ in $\mathbb{H}^{3} \cup \partial_{\infty}\left(\mathbb{H}^{3}\right)$, and $\mathcal{F}_{N}=\mathbb{C}$, the claim follows.

Now suppose that $\infty$ is a bounded parabolic fixed point of rank one. Let $n_{v_{1}} \in$ $\Gamma \cap N$ be a generator for $\Gamma \cap N$ for $v_{1} \in \mathbb{C}$, and fix a vector $v_{2} \in \mathbb{C}$ perpendicular to $v_{1}$. The set $\mathcal{F}_{N}:=\left\{s_{1} v_{1}+s_{2} v_{2} \in \mathbb{C}: 0 \leq s_{1}<1\right\}$ is a fundamental domain in $\mathbb{C}$ for the action of $\Gamma \cap N$. By replacing $\mathcal{F}$ if necessary, we may assume that $\mathcal{F} \subset \mathcal{F}_{N} \times \mathbb{R}_{>0}$. Then by [26, Prop. A. 14 in VI], for all large $c>1$, the sets

$$
S(c):=\left\{\left(s_{1} v_{1}+s_{2} v_{2}, y\right) \in \mathbb{H}^{3}: y>c \text { or }\left|s_{2}\right|>c\right\}
$$

are precisely invariant by $\Gamma \cap N$ and $\mathcal{F}-S(c)$ is bounded away from $\infty$. Since $\mathcal{F} \cap S(c) \neq \emptyset$ as $\infty \in \overline{\mathcal{F}}$ and $S(c)$ is precisely invariant by $N \cap \Gamma$, it follows that

$$
\left\{\left(x_{1}, x_{2}, y\right) \in S(c): x_{1}+i x_{2} \in \mathcal{F}_{N}\right\} \subset \mathcal{F} \text {. }
$$


Since

$$
\left\{\left(x_{1}, x_{2}, y\right): x_{1}+i x_{2} \in \mathcal{F}_{N}, x_{1}^{2}+x_{2}^{2}+y^{2}>r\right\} \subset S(c)
$$

for some large $c>0$, the claim follows in this case.

If $\infty$ is a bounded parabolic point of rank two, $S(c):=\left\{\left(x_{1}, x_{2}, y\right): y>c\right\}$ is precisely invariant by $\Gamma \cap N$ and $\mathcal{F}-S(c)$ is bounded away from $\infty$ by 26. Prop. A. 13 in VI]. Choose $\mathcal{F}_{N}$ so that $\mathcal{F} \subset \mathcal{F}_{N} \times \mathbb{R}_{>0}$. Then (3.6) holds for the same reason, and since $\mathcal{F}_{N}$ is bounded,

$$
\left\{\left(x_{1}, x_{2}, y\right): x_{1}+i x_{2} \in \mathcal{F}_{N}, x_{1}^{2}+x_{2}^{2}+y^{2}>r\right\} \subset S(c)
$$

for some large $c>0$. This proves the claim.

Considering the action of $N \cap \Gamma$ on $\partial_{\infty}\left(\mathbb{H}^{3}\right) \backslash\{\infty\}=\mathbb{C}$, we denote by $\Lambda_{N}(\Gamma)$ the image of $\Lambda(\Gamma) \backslash\{\infty\}$ in the quotient $(N \cap \Gamma) \backslash \mathbb{C}$, that is,

$$
\Lambda_{N}(\Gamma):=\left\{\left[n_{x}\right] \in(N \cap \Gamma) \backslash N: x \in \Lambda(\Gamma) \backslash\{\infty\}\right\} .
$$

Proposition 3.7. If $\Gamma \backslash \Gamma N$ is closed, the set $\Lambda_{N}(\Gamma)$ is bounded.

Proof. This is clear if $\infty$ is a bounded parabolic point of rank two, as $N \cap \Gamma \backslash N$ is compact. If $\infty \notin \Lambda(\Gamma)$, then $\Lambda(\Gamma)$ is a compact subset of $\mathbb{C}$, and hence the claim follows. In the case when $\infty$ is a bounded parabolic point of rank one, the claim follows from the well-known fact that $\Lambda(\Gamma)$ lies in a strip of finite width (see [47, proof of Prop. 8.4.3]). This can also be deduced from Proposition 3.5 using the fact that the intersection of the convex core of $\Gamma \backslash \mathbb{H}^{3}$ and the thick part of the manifold $\Gamma \backslash \mathbb{H}^{3}$ is compact for a geometrically finite group.

Proposition 3.8. Assume that $\Gamma \backslash \Gamma N$ is closed. For any compact subset $J \subset \Gamma \backslash G$, the following set is bounded:

$$
N(J):=\{[n] \in(N \cap \Gamma) \backslash N: \Gamma \backslash \Gamma n A \cap J \neq \emptyset\} .
$$

Proof. Let $\mathcal{F}_{N}$ and $\mathcal{F}$ be as in Proposition 3.5. If $\mathcal{F}_{N}$ is bounded, there is nothing to prove. Hence by Theorem [3.3, we may assume that either $\infty \notin \Lambda(\Gamma)$ or $\infty$ is a bounded parabolic fixed point of rank one.

To prove the proposition, suppose on the contrary that there exist sequences $n_{j} \in \mathcal{F}_{N} \rightarrow \infty, a_{j} \in A, \gamma_{j} \in \Gamma$ and $w_{j} \in J$ such that $n_{j} a_{j}=\gamma_{j} w_{j}$. As $J$ is bounded, we may assume $w_{j} \rightarrow w \in G$ by passing to a subsequence. Then $\gamma_{j}^{-1} n_{j} a_{j} \rightarrow w$. Let $\gamma_{0} \in \Gamma$ be such that $\gamma_{0} w(0,0,1) \in \overline{\mathcal{F}}$. By the geometric finiteness of $\Gamma$, there exists a finite union, say $\mathcal{F}^{\prime}$, of translates of $\mathcal{F}$ such that $\gamma_{0} w_{j}(0,0,1) \in \mathcal{F}^{\prime}$ for all large $j$.

As $n_{j} \rightarrow \infty$, the Euclidean norm of $n_{j} a_{j}(0,0,1)$ goes to infinity, and hence by Proposition 3.5 .

$$
n_{j} a_{j}(0,0,1) \in \mathcal{F}^{\prime} \quad \text { for all large } j
$$

by enlarging $\mathcal{F}^{\prime}$ if necessary.

Therefore for all large $j$,

$$
\gamma_{0} \gamma_{j}^{-1} n_{j} a_{j}(0,0,1)=\gamma_{0} w_{j}(0,0,1) \in \mathcal{F}^{\prime} \cap \gamma_{0} \gamma_{j}^{-1}\left(\mathcal{F}^{\prime}\right) .
$$

Since $\mathcal{F}^{\prime} \cap \gamma_{0} \gamma_{j}^{-1}\left(\mathcal{F}^{\prime}\right) \neq \emptyset$ for only finitely many $\gamma_{j}$ 's, we conclude that $\left\{\gamma_{j}\right\}$ must be a finite set. As $n_{j} a_{j}=\gamma_{j} w_{j} \in \gamma_{j} J, n_{j} a_{j}$ must be a bounded sequence, contradicting $n_{j} \rightarrow \infty$. 
Note that $N(J)$ is defined so that for all $y>0$,

$$
(N \cap \Gamma) \backslash N a_{y} \cap J \subset N(J) .
$$

Corollary 3.10. Let $\psi \in C_{c}(\Gamma \backslash G)$ have support $J$.

(1) The set $N(J)$ defined in (3.9) is bounded.

(2) For any function $\eta \in C_{c}(N \cap \Gamma \backslash N)$ with $\left.\eta\right|_{N(J)} \equiv 1$, we have for all $y>0$,

$$
\int_{(N \cap \Gamma) \backslash N} \psi\left(n a_{y}\right) d n=\int_{(N \cap \Gamma) \backslash N} \psi\left(n a_{y}\right) \eta(n) d n .
$$

Proof. The first claim is immediate from the above proposition. For (2), it suffices to note that $\psi\left(n a_{y}\right) \equiv 0$ for $n$ outside of $N(J)$, and hence

$$
\int_{(N \cap \Gamma) \backslash N} \psi\left(n a_{y}\right) \eta(n) d n=\int_{N(J)} \psi\left(n a_{y}\right) \eta(n) d n .
$$

Using $\eta \equiv 1$ on $N(J)$, the claim follows.

\section{The Base EigenfunCtion $\phi_{0}$}

In this section, we assume that $\Gamma<G=\mathrm{PSL}_{2}(\mathbb{C})$ is a geometrically finite torsion-free discrete subgroup and that the Hausdorff dimension $\delta_{\Gamma}$ of the limit set $\Lambda(\Gamma)$ is strictly greater than one. Assume also that $\Gamma \backslash \Gamma N$ is closed.

By Sullivan [45], there exists a positive $L^{2}$-eigenfunction $\phi_{0}$, unique up to a scalar multiple, of the Laplace operator $\Delta$ on $\Gamma \backslash \mathbb{H}^{3}$ with smallest eigenvalue $\delta_{\Gamma}\left(2-\delta_{\Gamma}\right)$ and which is square-integrable, that is,

$$
\int_{\Gamma \backslash \mathbb{H}^{3}} \phi_{0}(x, y)^{2} \frac{1}{y^{3}} d x d y<\infty .
$$

In this section, we study the properties of $\phi_{0}$ along closed horospheres. For the base point $o=(0,0,1)$, we denote by $\nu_{o}$ a weak limit as $s \rightarrow \delta_{\Gamma}$ of the family of measures on $\overline{\mathbb{H}}^{3}$ :

$$
\nu(s):=\frac{1}{\sum_{\gamma \in \Gamma} e^{-s d(o, \gamma o)}} \sum_{\gamma \in \Gamma} e^{-s d(o, \gamma o)} \delta_{\gamma o} .
$$

The measure $\nu_{o}$ is indeed the unique weak limit of $\{\nu(s)\}$ as $s \rightarrow \delta_{\Gamma}$. Sullivan [45] showed that $\nu_{o}$ is the unique finite measure (up to a constant multiple) supported on $\Lambda(\Gamma)$ with the property: for any $\gamma \in \Gamma$,

$$
\frac{d\left(\gamma_{*} \nu_{o}\right)}{d \nu_{o}}(\xi)=e^{-\delta_{\Gamma} \beta_{\xi}(\gamma(o), o)},
$$

where $\beta_{\xi}\left(z_{1}, z_{2}\right):=\lim _{z \rightarrow \xi} d\left(z_{1}, z\right)-d\left(z_{2}, z\right)$ is the Busemann function and $\gamma_{*} \nu_{o}(A)$ $:=\nu_{o}\left(\gamma^{-1}(A)\right)$. The measure $\nu_{o}$, called the Patterson-Sullivan measure, has no atoms.

The base eigenfunction $\phi_{0}$ can be explicitly written as the integral of the Poisson kernel against $\nu_{o}$ :

$$
\begin{aligned}
\phi_{0}(x+i y) & =\int_{u \in \Lambda(\Gamma)} e^{-\delta_{\Gamma} \beta_{u}(x+i y, o)} d \nu_{o}(u) \\
& =\int_{u \in \Lambda(\Gamma) \backslash\{\infty\}}\left(\frac{\left(\|u\|^{2}+1\right) y}{\|x-u\|^{2}+y^{2}}\right)^{\delta_{\Gamma}} d \nu_{o}(u),
\end{aligned}
$$


where $\Lambda(\Gamma)$ is identified as a subset of $\mathbb{C} \cup\{\infty\}$. The main goal of this section is to study the average of $\phi_{0}$ along the translates $\Gamma \backslash \Gamma N a_{y}$.

Definition 4.4. For a given $\psi \in C(\Gamma \backslash G)^{K}$, define

$$
\psi^{N}\left(a_{y}\right):=\int_{n \in(N \cap \Gamma) \backslash N} \psi\left(n a_{y}\right) d n .
$$

We will show that the integral $\phi_{0}^{N}\left(a_{y}\right)$ above converges, and moreover that all of the action in this integral takes place only over the following bounded set (see Proposition 3.7):

$$
\Lambda_{N}(\Gamma)=\left\{\left[n_{x}\right] \in(N \cap \Gamma) \backslash N: x \in \Lambda(\Gamma) \backslash\{\infty\}\right\} .
$$

If $\infty$ is a bounded parabolic fixed point of rank one, then there exists a vector $v_{1} \in \mathbb{R}^{2}$ such that $n_{v_{1}}$ is a generator for $N \cap \Gamma$. Fixing vector $v_{2}$ perpendicular to $v_{1}$, we can decompose $x \in \mathbb{R}^{2}$ as $x=x_{1} v_{1}+x_{2} v_{2}$.

Definition 4.5. We say that an open subset $B \subset(N \cap \Gamma) \backslash N$ properly covers $\Lambda_{N}(\Gamma)$ if the following hold:

(1) if $\infty \notin \Lambda(\Gamma)$, then $\epsilon_{0}(B):=\inf _{u \in \Lambda_{N}(\Gamma), x \notin B}\|x-u\|>0$;

(2) if $\infty$ is a bounded parabolic fixed point of rank one, then

$$
\epsilon_{0}(B):=\inf _{x \notin B, u \in \Lambda_{N}(\Gamma)}\left|u_{2}-x_{2}\right|>0 ;
$$

(3) if $\infty$ is a bounded parabolic fixed point of rank two, then $B=(N \cap \Gamma) \backslash N$.

Proposition 4.6. (1) $\phi_{0}^{N}\left(a_{y}\right) \gg y^{2-\delta_{\Gamma}}$ for all $0<y \ll 1$.

(2) For any open subset $B \subset(N \cap \Gamma) \backslash N$ which properly covers $\Lambda_{N}(\Gamma)$, and all small $y>0$,

$$
\phi_{0}^{N}\left(a_{y}\right)=\int_{B} \phi_{0}\left(n_{x} a_{y}\right) d x+O_{\epsilon_{0}(B)}\left(y^{\delta_{\Gamma}}\right) .
$$

Proof. Choose a fundamental domain $\mathcal{F}_{N} \subset \mathbb{C}$ for $(N \cap \Gamma) \backslash N$. We first show that $\phi_{0}^{N}\left(a_{y}\right) \gg y^{2-\delta_{\Gamma}}$ for all $0<y \ll 1$. For a subset $Q \subset \mathcal{F}_{N}$, the notation $Q^{c}$ denotes the complement of $Q$ in $\mathcal{F}_{N}$. Observe that for a subset $Q \subset \mathcal{F}_{N}$,

$$
\begin{aligned}
& \int_{x \in Q} \phi_{0}\left(n_{x} a_{y}\right) d x \\
& =\int_{u \in \Lambda(\Gamma)}\left(\|u\|^{2}+1\right)^{\delta_{\Gamma}} \int_{x \in Q}\left(\frac{y}{\|x-u\|^{2}+y^{2}}\right)^{\delta_{\Gamma}} d x d \nu_{o}(u) \\
& =\int_{u \in \Lambda(\Gamma)}\left(\int_{x \in Q-u}\left(\|u\|^{2}+1\right)^{\delta_{\Gamma}}\left(\frac{y}{\|x\|^{2}+\|y\|^{2}}\right)^{\delta_{\Gamma}} d x\right) d \nu_{o}(u),
\end{aligned}
$$

where the interchange of orders is justified since everything is non-negative.

We observe that by changing the variable $w=\frac{x}{y}$,

$$
\begin{aligned}
\int_{x \in \mathbb{R}^{2}}\left(\frac{y}{\|x\|^{2}+\|y\|^{2}}\right)^{\delta_{\Gamma}} d x & =y^{2-\delta_{\Gamma}} \int_{w \in \mathbb{R}^{2}}\left(\frac{1}{\|w\|^{2}+1}\right)^{\delta_{\Gamma}} d w \\
& =y^{2-\delta_{\Gamma}} 2 \pi \int_{r>0} \frac{r}{\left(r^{2}+1\right)^{\delta_{\Gamma}}} d r \\
& =\frac{\pi}{\delta_{\Gamma}-1} y^{2-\delta_{\Gamma}} .
\end{aligned}
$$


Case I: $\infty \notin \Lambda(\Gamma)$. In this case, we have

$$
\omega_{0}=\int_{u \in \Lambda(\Gamma)}\left(\|u\|^{2}+1\right)^{\delta_{\Gamma}} d \nu_{o}(u) \ll \nu_{o}(\Lambda(\Gamma))<\infty .
$$

Therefore for $Q=\mathbb{C}$, we obtain from (4.8) and (4.9) that

$$
\begin{aligned}
\phi_{0}^{N}\left(a_{y}\right) & =\int_{u \in \Lambda(\Gamma)}\left(\|u\|^{2}+1\right)^{\delta_{\Gamma}} d \nu_{o}(u) \cdot \int_{x \in \mathbb{R}^{2}}\left(\frac{y}{\|x\|^{2}+\|y\|^{2}}\right)^{\delta_{\Gamma}} d x \\
& =\frac{\omega_{0} \cdot \pi}{\delta_{\Gamma}-1} y^{2-\delta_{\Gamma}} .
\end{aligned}
$$

This proves (1). To prove (2), let $B$ be an open subset of $\mathbb{C}$ which properly covers $\Lambda_{N}(\Gamma)$. Then we have

$$
\epsilon_{0}:=\inf _{u \in \Lambda_{N}(\Gamma), x \in B^{c}}\|x-u\|=\inf \left\{\|x\|: x \in B^{c}-\Lambda_{N}(\Gamma)\right\}>0 .
$$

By setting $Q=B^{c}$ in (4.8), we deduce

$$
\begin{aligned}
\int_{x \in B^{c}} \phi_{0}\left(n_{x} a_{y}\right) d x & \leq \omega_{0} \cdot \int_{\|x\|>\epsilon_{0}}\left(\frac{y}{\|x\|^{2}+\|y\|^{2}}\right)^{\delta_{\Gamma}} d x \\
& \leq \omega_{0} y^{2-\delta_{\Gamma}} \cdot \int_{\|w\|>\epsilon_{0} y^{-1}}\left(\frac{1}{\|w\|^{2}+1}\right)^{\delta_{\Gamma}} d w \\
& =2 \pi \omega_{0} y^{2-\delta_{\Gamma}} \cdot \int_{r>\epsilon_{0} y^{-1}} \frac{r}{\left(r^{2}+1\right)^{\delta_{\Gamma}}} d r \\
& =2 \pi \omega_{0} y^{2-\delta_{\Gamma}}\left(\frac{\epsilon_{0}^{2}}{y^{2}}+1\right)^{1-\delta_{\Gamma}} \\
& \ll y^{\delta_{\Gamma}} .
\end{aligned}
$$

Case II: $\infty$ is a bounded parabolic fixed point of rank one.

We may assume without loss of generality that $\Gamma \cap N$ is generated by $(1,0)$, that is, $x \mapsto x+1$, and $\mathcal{F}_{N}=\left\{\left(x_{1}, x_{2}\right): 0 \leq x_{1}<1, x_{2} \in \mathbb{R}\right\}$. There exists $T>0$ such that $\Lambda(\Gamma) \subset \mathbb{R} \times[-T, T]$ by [47, proof of Prop. 8.4.3].

By computing the Busemann function, we deduce from (4.1) for $k=\left(k_{1}, k_{2}\right) \in$ $\Gamma \cap N$ that

$$
d\left(\left(n_{-k}\right)_{*} \nu_{o}\right)(u)=\left(\frac{\|u\|^{2}+1}{\|k-u\|^{2}+1}\right)^{\delta_{\Gamma}} d \nu_{o}(u) .
$$

We have by changing orders of integrations of $x_{1}$ and $u_{1}$ that

$$
\begin{aligned}
& \int_{x \in \mathcal{F}_{N}} \phi_{0}(x, y) d x \\
& =\int_{u \in \mathbb{R} \times[-T, T]} \int_{x \in([0,1] \times \mathbb{R})-u}\left(\frac{\left(\|u\|^{2}+1\right) y}{\|x\|^{2}+y^{2}}\right)^{\delta_{\Gamma}} d x d \nu_{o}(u) \\
& =\int_{x \in \mathbb{R}^{2}}\left(\frac{y}{\|x\|^{2}+y^{2}}\right)^{\delta_{\Gamma}} \int_{u \in\left[-x_{1}, 1-x_{1}\right] \times[-T, T]}\left(\|u\|^{2}+1\right)^{\delta_{\Gamma}} d \nu_{o}(u) d x .
\end{aligned}
$$

We set $c_{1}$ and $c_{2}$ to be, respectively, the infimum and the supremum of

$$
e_{x_{1}}:=\int_{u \in\left[-x_{1}, 1-x_{1}\right] \times[-T, T]}\left(\|u\|^{2}+1\right)^{\delta_{\Gamma}} d \nu_{o}(u)
$$


over all $x_{1} \in \mathbb{R}$. We claim that

$$
0<c_{1} \leq c_{2}<\infty
$$

For any $k_{1} \in \mathbb{Z}$, by changing the variable $u_{1} \mapsto u_{1}+k_{1}$ and recalling (4.11), we deduce

$$
\begin{aligned}
e_{x_{1}}= & \int_{u \in\left[-x_{1}, 1-x_{1}\right] \times[-T, T]}\left(\|u\|^{2}+1\right)^{\delta_{\Gamma}} d \nu_{o}(u) \\
& =\int_{u_{1}=k_{1}-x_{1}}^{u_{1}=k_{1}+1-x_{1}} \int_{u_{2}=-T}^{u_{2}=T}\left(\|u-k\|^{2}+1\right)^{\delta_{\Gamma}} d\left(\left(n_{-k}\right)_{*} \nu_{o}\right)(u) \\
& =\int_{u_{1}=k_{1}-x_{1}}^{u_{1}=k_{1}+1-x_{1}} \int_{u_{2}=-T}^{u_{2}=T}\left(\|u\|^{2}+1\right)^{\delta_{\Gamma}} d \nu_{o}(u) .
\end{aligned}
$$

Choosing $k_{1}$ so that $x_{1} \in\left[k_{1}-1, k_{1}\right)$, we have $e_{x_{1}} \ll \nu_{o}([0,2] \times[-T, T])$ and hence $c_{2}<\infty$. Note that $e_{x_{1}} \asymp e_{x_{1}+1}$, where the implied constant is independent of $x_{1} \in \mathbb{R}$, and that

$$
e_{x_{1}}+e_{x_{1}+1}=\int_{u_{1}=k_{1}-x_{1}-1}^{u_{1}=k_{1}+1-x_{1}} \int_{u_{2}=-T}^{u_{2}=T}\left(\|u\|^{2}+1\right)^{\delta_{\Gamma}} d \nu_{o}(u) \gg \nu_{o}([0,1] \times[-T, T]) .
$$

On the other hand, since the $N \cap \Gamma$-translates of $[0,1] \times[-T, T]$ cover the support of $\nu_{o}$ except for $\infty$ and $\nu_{o}$ is atom-free, we have $\nu_{o}([0,1] \times[-T, T])>0$. This proves that $c_{1}=\inf e_{x_{1}}>0$.

We now deduce from (4.12) and (4.9) that

$$
\int_{x \in \mathcal{F}_{N}} \phi_{0}(x, y) d x \gg c_{1} \int_{x \in \mathbb{R}^{2}}\left(\frac{y}{\|x\|^{2}+y^{2}}\right)^{\delta_{\Gamma}} d x \gg y^{2-\delta_{\Gamma}},
$$

proving (1).

If $B$ is an open subset of $\mathcal{F}_{N}=[0,1] \times \mathbb{R}$ which properly covers $\Lambda_{N}(\Gamma)$, we have

$$
\epsilon_{0}:=\inf _{x \in B^{c}, u \in \Lambda(\Gamma)}\left|u_{2}-x_{2}\right|>0 .
$$

Hence $\left\{(x, u) \in \mathbb{R}^{2} \times \Lambda_{N}(\Gamma): x \in B^{c}-u\right\}$ is contained in the set

$$
\left\{u_{1} \in \mathbb{R},-u_{1}<x_{1}<1-u_{1},\left|u_{2}\right|<T,\left|x_{2}\right|>\epsilon_{0}\right\} .
$$

Since

$$
\left\{u_{1} \in \mathbb{R}, \quad-u_{1}<x_{1}<1-u_{1}\right\}=\left\{x_{1} \in \mathbb{R}, \quad-x_{1}<u_{1}<1-x_{1}\right\},
$$

we deduce from (4.8) by changing the order of integrations that

$$
\begin{aligned}
& \int_{x \in B^{c}} \phi_{0}(x, y) d x \\
& =\int_{u \in \Lambda(\Gamma)} \int_{x \in B^{c}-u}\left(\frac{\left(\|u\|^{2}+1\right) y}{\|x\|^{2}+y^{2}}\right)^{\delta_{\Gamma}} d x d \nu_{o}(u) \\
& \leq \int_{x_{1} \in \mathbb{R},\left|x_{2}\right|>\epsilon_{0}}\left(\frac{y}{\|x\|^{2}+y^{2}}\right)^{\delta_{\Gamma}}\left(\int_{u_{1}=-x_{1}}^{u_{1}=1-x_{1}} \int_{u_{2}=-T}^{u_{2}=T}\left(\|u\|^{2}+1\right)^{\delta_{\Gamma}} d \nu_{o}(u)\right) d x_{2} d x_{1} \\
& \leq c_{2} \cdot \int_{x_{1} \in \mathbb{R},\left|x_{2}\right|>\epsilon_{0}}\left(\frac{y}{\|x\|^{2}+y^{2}}\right)^{\delta_{\Gamma}} d x_{2} d x_{1} .
\end{aligned}
$$


The $x_{1}$ integral can be evaluated explicitly and yields:

$$
\begin{aligned}
\int_{x \in B^{c}} \phi_{0}(x, y) d x & \ll \frac{\sqrt{\pi} \cdot \Gamma\left(\delta_{\Gamma}-1 / 2\right)}{\Gamma\left(\delta_{\Gamma}\right)} y^{\delta_{\Gamma}} \int_{\left|x_{2}\right|>\epsilon_{0}}\left(\frac{1}{x_{2}^{2}+y^{2}}\right)^{\delta_{\Gamma}-1 / 2} d x_{2} \\
& \ll \frac{\sqrt{\pi} \cdot \Gamma\left(\delta_{\Gamma}-1 / 2\right)}{\Gamma\left(\delta_{\Gamma}\right)} y^{\delta_{\Gamma}} \int_{\left|x_{2}\right|>\epsilon_{0}}\left(\frac{1}{x_{2}^{2}}\right)^{\delta_{\Gamma}-1 / 2} d x_{2} \\
& \ll y^{\delta_{\Gamma}} .
\end{aligned}
$$

Hence (2) is proved.

Case III: $\infty$ is a bounded parabolic fixed point of rank two. In this case (2) holds for a trivial reason. But we still need to show (1). The argument is similar to case II. Without loss of generality, we assume that $N \cap \Gamma$ is generated by $(1,0)$ and $(0,1)$, so that $\mathcal{F}_{N}=[0,1] \times[0,1]$.

Similarly to (4.12) we have

$$
\phi_{0}^{N}\left(a_{y}\right)=\int_{x \in \mathbb{R}^{2}}\left(\frac{y}{\|x\|^{2}+y^{2}}\right)^{\delta_{\Gamma}} \int_{u \in\left[-x_{1}, 1-x_{1}\right] \times\left[-x_{2}, 1-x_{2}\right]}\left(\|u\|^{2}+1\right)^{\delta_{\Gamma}} d \nu_{o}(u) d x .
$$

By (4.9), it suffices to show that

$$
c_{0}:=\inf _{x_{1}, x_{2} \in \mathbb{R}} \int_{u \in\left[-x_{1}, 1-x_{1}\right] \times\left[-x_{2}, 1-x_{2}\right]}\left(\|u\|^{2}+1\right)^{\delta_{\Gamma}} d \nu_{o}(u)>0 .
$$

For $\left(x_{1}, x_{2}\right) \in\left[k_{1}-1, k_{1}\right) \times\left[k_{2}-1, k_{2}\right)$, by changing the variables $u_{i} \rightarrow u_{i}+k_{i}$ for $k_{i} \in \mathbb{Z}$ and by using (4.11), we deduce

$$
\begin{aligned}
\int_{u_{1}=-x_{1}}^{u_{1}=1-x_{1}} \int_{u_{2}=-x_{2}}^{u_{2}=1-x_{2}}\left(\|u\|^{2}+1\right)^{\delta_{\Gamma}} d \nu_{o}(u) & \\
& =\int_{u_{1}=k_{1}-x_{1}}^{u_{1}=k_{1}+1-x_{1}} \int_{u_{2}=k_{2}-x_{2}}^{u_{2}=k_{2}+1-x_{2}}\left(\|u\|^{2}+1\right)^{\delta_{\Gamma}} d \nu_{o}(u) .
\end{aligned}
$$

Hence

$$
c_{0} \gg \inf _{k_{i}-1 \leq x_{i} \leq k_{i}} \nu_{o}\left(\left[k_{i}-x_{i}, k_{i}-x_{i}+1-x_{i}\right]\right) \gg \nu_{o}\left([0,1]^{2}\right)>0
$$

Corollary 4.13. For any $y>0$, there exist $c_{\phi_{0}}>0$ and $d_{\phi_{0}} \geq 0$ such that

$$
\phi_{0}^{N}\left(a_{y}\right)=c_{\phi_{0}} y^{2-\delta_{\Gamma}}+d_{\phi_{0}} y^{\delta_{\Gamma}} .
$$

If $\infty \notin \Lambda(\Gamma)$, then $d_{\phi_{0}}=0$.

Proof. Since $\Delta \phi_{0}=\delta_{\Gamma}\left(2-\delta_{\Gamma}\right) \phi_{0}$, it follows that

$$
-y^{2} \frac{\partial^{2}}{\partial y^{2}} \phi_{0}^{N}+y \frac{\partial}{\partial y} \phi_{0}^{N}=\delta_{\Gamma}\left(2-\delta_{\Gamma}\right) \phi_{0}^{N} .
$$

As both $y^{\delta_{\Gamma}}$ and $y^{2-\delta_{\Gamma}}$ satisfy the above differential equation, we have

$$
\phi_{0}^{N}\left(a_{y}\right)=c_{\phi_{0}} y^{2-\delta_{\Gamma}}+d_{\phi_{0}} y^{\delta_{\Gamma}}
$$

for some $c_{\phi_{0}}, d_{\phi_{0}} \geq 0$. Proposition 4.6 (1) implies that $c_{\phi_{0}}>0$.

In the case when $\mathcal{F}_{N}=\mathbb{C}$, the last claim is proved in (4.10). 


\section{SPHERICAL FUNCTIONS AND SPECTRAL BOUNDS}

We keep the notation set up in section 3 . Let $0 \leq s \leq 2$ and consider the character $\chi_{s}$ on the subgroup $B:=A M N$ of $G$ defined by

$$
\chi_{s}\left(a_{y} m n\right)=y^{s}
$$

where $a_{y} \in A$ is given as before, and $m \in M$ and $n \in N$.

The unitarily induced representation $\left(\pi_{s}:=\operatorname{Ind}_{B}^{G} \chi_{s}, V_{s}\right)$ admits a unique $K$ invariant unit vector, say $v_{s}$.

By the theory of spherical functions,

$$
f_{s}(g):=\left\langle\pi_{s}(g) v_{s}, v_{s}\right\rangle=\int_{K} v_{s}(k g) d k
$$

is the unique bi- $K$-invariant function of $G$ with $f_{s}(e)=1$ and with $\mathcal{C} f_{s}=s(2-s) f_{s}$, where $\mathcal{C}$ is the Casimir operator of $G$. Moreover, there exist $c_{s}>0$ and $\epsilon>0$ such that for all $y$ small,

$$
f_{s}\left(a_{y}\right)=c_{s} \cdot y^{2-s}\left(1+O\left(y^{\epsilon}\right)\right)
$$

by [14, 4.6].

Since the Casimir operator is equal to the Laplace operator $\Delta$ on $K$-invariant functions, this implies:

Theorem 5.2. Let $\Gamma<G$ be a discrete subgroup. Let $\phi_{s} \in L^{2}(\Gamma \backslash G)^{K} \cap C^{\infty}(\Gamma \backslash G)$ satisfy $\Delta \phi_{s}=s(2-s) \phi_{s}$ and $\left\|\phi_{s}\right\|_{2}=1$. Then there exist $c_{s}>0$ and $\epsilon>0$ such that for all small $0<y<1$,

$$
\left\langle a_{y} \phi_{s}, \phi_{s}\right\rangle_{L^{2}(\Gamma \backslash G)}=c_{s} \cdot y^{2-s}\left(1+O\left(y^{\epsilon}\right)\right) .
$$

In the unitary dual of $G$, the spherical part consists of the principal series and the complementary series. We use the parametrization of $s \in\{1+i \mathbb{R}\} \cup[1,2]$ so that $s=$ 2 corresponds to the trivial representation and the vertical line $1+i \mathbb{R}$ corresponds to the tempered spectrum. Then the complementary series is parametrized by $V_{s}$, $1<s \leq 2$, defined before.

Let $\left\{X_{i}\right\}$ denote an orthonormal basis of the Lie algebra of $K$ with respect to an $A d$-invariant scalar product, and define $\omega:=1-\sum X_{i}^{2}$. This is a differential operator in the center of the enveloping algebra of Lie $(K)$ and acts as a scalar on each $K$-isotypic component of $V_{s}$.

Proposition 5.3. Fix $1<s_{0}<2$. Let $(V, \pi)$ be a representation of $G$ which does not weakly contain any complementary series representation $V_{s}$ with parameter $s \geq s_{0}$. Then for any $\epsilon>0$, there exists $c_{\epsilon}>0$ such that for any smooth vectors $w_{1}, w_{2} \in V$, and $y<1$,

$$
\left|\left\langle a_{y} w_{1}, w_{2}\right\rangle\right| \leq c_{\epsilon} \cdot y^{2-s_{0}-\epsilon} \cdot\left\|\omega\left(w_{1}\right)\right\| \cdot\left\|\omega\left(w_{2}\right)\right\| .
$$

Proof. (We refer to 42 for the arguments below.) As a $G$-representation, $\pi$ has a Hilbert integral decomposition $\pi=\int_{z \in \hat{G}} \oplus^{m_{z}} \rho_{z} d \nu(z)$, where $\hat{G}$ denotes the unitary dual of $G, \rho_{z}$ is irreducible and $m_{z}$ is the multiplicity of $\rho_{z}$, and $\nu$ is the spectral measure on $\hat{G}$. By the assumption on $\pi$, for almost all $z, \rho_{z}$ is either tempered or isomorphic to $\pi_{s}$ for $1 \leq s \leq s_{0}$. As $1<3-s_{0}<2$, there exists the complementary series $\left(V_{3-s_{0}}, \pi_{3-s_{0}}\right)$. We claim that the tensor product $\rho_{z} \otimes \pi_{3-s_{0}}$ is a tempered representation. Recall that a unitary representation of $G$ is tempered if and only if there exists a dense subset of vectors whose matrix coefficients are $L^{2+\epsilon}$-integrable 
for any $\epsilon>0$. If $\rho_{z}$ is tempered, $\rho_{z} \otimes \pi_{3-s_{0}}$ is clearly tempered. If $\rho_{z}$ is isomorphic to $\pi_{s}$ for some $1 \leq s \leq s_{0}$, and $v_{z}$ denotes the spherical vector of $\rho_{z}$ of norm one,

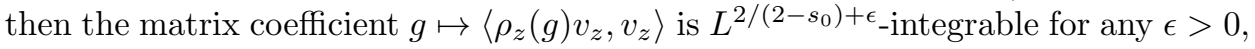
by (5.1) together with the fact that the Haar measure on $G$ satisfies $d\left(k_{1} a_{y} k_{2}\right) \asymp$ $y^{-3} d k_{1} d y d k_{2}$ for all $0<y \leq 1$. Since $\rho_{z}$ is irreducible, it follows that there exists a dense set of vectors whose matrix coefficients are $L^{2 /\left(2-s_{0}\right)+\epsilon}$-integrable for any $\epsilon>0$. Similarly there exists a dense set of vectors in $V_{3-s_{0}}$ whose matrix coefficients are $L^{2 /\left(s_{0}-1\right)+\epsilon}$-integrable for any $\epsilon>0$. Hence by the Hölder inequality, there exists a dense set of vectors in $\rho_{z} \otimes \pi_{3-s_{0}}$ whose matrix coefficients are $L^{2+\epsilon}$-integrable for any $\epsilon>0$, implying that $\rho_{z} \otimes \pi_{3-s_{0}}$ is tempered.

Since $\pi \otimes \pi_{3-s_{0}}=\int_{z} \oplus^{m_{z}}\left(\rho_{z} \otimes \pi_{3-s_{0}}\right) d \nu(z)$, we deduce that $\pi \otimes \pi_{3-s_{0}}$ is tempered.

We now claim that for any $\epsilon>0$, there is a constant $c_{\epsilon}>0$ such that for any $K$-finite unit vectors $w_{1}$ and $w_{2}$, we have

$$
\left|\left\langle a_{y} w_{1}, w_{2}\right\rangle\right| \leq c_{\epsilon} \cdot y^{2-s_{0}-\epsilon} \cdot \prod_{i} \sqrt{\left(\operatorname{dim}\left\langle K w_{i}\right\rangle\right)} .
$$

Noting that the $K$-span of $w_{i} \otimes v_{3-s_{0}}$ has the same dimension as the $K$-span of $w_{i}$, the temperedness of $\pi \otimes \pi_{3-s_{0}}$ implies that for any $\epsilon>0$, there exists a constant $c_{\epsilon}>0$ such that

$$
\begin{aligned}
\left\langle a_{y}\left(w_{1} \otimes v_{3-s_{0}}\right),\left(w_{2} \otimes v_{3-s_{0}}\right)\right\rangle & =\left\langle a_{y} w_{1}, w_{2}\right\rangle \cdot\left\langle a_{y} v_{3-s_{0}}, v_{3-s_{0}}\right\rangle \\
& \leq c_{\epsilon} \cdot y^{1+\epsilon} \cdot \prod_{i} \sqrt{\left(\operatorname{dim}\left\langle K w_{i}\right\rangle\right)}
\end{aligned}
$$

As $\left\langle a_{y} v_{3-s_{0}}, v_{3-s_{0}}\right\rangle=c \cdot y^{-1+s_{0}}\left(1+O\left(y_{0}^{\epsilon}\right)\right)$ for some $\epsilon_{0}>0$, the claim (5.4) follows. Passing from the above bounds of (6.6) for $K$-finite vectors to those for smooth vectors has been detailed in [28, proof of Thm. 6]. In particular, in the case of $G=\mathrm{SL}_{2}(\mathbb{C})$, the above degree of Sobolev norm suffices.

Definition 5.5. For a geometrically finite discrete subgroup $\Gamma$ of $G$ with $\delta_{\Gamma}>1$, we fix $1<s_{\Gamma}<\delta_{\Gamma}$ so that there is no eigenvalue of $\Delta$ between $s_{\Gamma}\left(2-s_{\Gamma}\right)$ and the base eigenvalue $\lambda_{0}=\delta_{\Gamma}\left(2-\delta_{\Gamma}\right)$ in $L^{2}(\Gamma \backslash G)$.

By the theorem of Lax-Phillips [24], the Laplace spectrum on $L^{2}(\Gamma \backslash G)^{K}$ has only finitely many eigenvalues outside the tempered spectrum. Therefore $1<s_{\Gamma}<\delta_{\Gamma}$ exists. The maximum difference between $\delta_{\Gamma}$ and $s_{\Gamma}$ will be referred to as the spectral gap for $\Gamma$.

Let $\left\{Z_{1}, \ldots, Z_{6}\right\}$ denote an orthonormal basis of the Lie algebra of $G$. Let $\Gamma<G$ be a discrete subgroup of $G$. For $f \in C^{\infty}(\Gamma \backslash G) \cap L^{2}(\Gamma \backslash G)$, we consider the following Sobolev norm $\mathcal{S}_{m}(f)$ :

$$
\mathcal{S}_{m}(f)=\max \left\{\left\|Z_{i_{1}} \cdots Z_{i_{\ell}}(f)\right\|_{2}: 1 \leq i_{j} \leq 6,0 \leq \ell \leq m\right\} .
$$

Corollary 5.6. Let $\Gamma$ be a geometrically finite discrete subgroup of $G$ with $\delta_{\Gamma}>1$. Then for any $\psi_{1} \in L^{2}(\Gamma \backslash G) \cap C^{\infty}(\Gamma \backslash G)^{K}, \psi_{2} \in C_{c}^{\infty}(\Gamma \backslash G)$ and $0<y<1$,

$$
\left\langle a_{y} \psi_{1}, \psi_{2}\right\rangle=\left\langle\psi_{1}, \phi_{0}\right\rangle\left\langle a_{y} \phi_{0}, \psi_{2}\right\rangle+O\left(y^{2-s_{\Gamma}} \mathcal{S}_{2}\left(\psi_{1}\right) \cdot \mathcal{S}_{2}\left(\psi_{2}\right)\right)
$$

Here $\phi_{0} \in L^{2}(\Gamma \backslash G)^{K}$ is the unique eigenfunction of $\Delta$ with eigenvalue $\delta_{\Gamma}\left(2-\delta_{\Gamma}\right)$ with unit $L^{2}$-norm.

Proof. We have

$$
L^{2}(\Gamma \backslash G)=W_{\delta_{\Gamma}} \oplus V
$$


where $W_{\delta_{\Gamma}}$ is isomorphic to $V_{\delta_{\Gamma}}$ as a $G$-representation and $V$ does not contain any complementary series $V_{s}$ with parameter $s>s_{\Gamma}$. Write $\psi_{1}=\left\langle\psi_{1}, \phi_{0}\right\rangle \phi_{0}+\psi_{1}^{\perp}$. Since $\phi_{0}$ is the unique $K$-invariant vector in $W_{\delta_{\Gamma}}$ up to a constant multiple, we have $\psi_{1}^{\perp} \in V^{K}$. Hence by Proposition [5.3, for any $\epsilon>0$ and $y \leq 1$,

$$
\begin{aligned}
\left\langle a_{y} \psi_{1}, \psi_{2}\right\rangle & =\left\langle\psi_{1}, \phi_{0}\right\rangle\left\langle a_{y} \phi_{0}, \psi_{2}\right\rangle+\left\langle a_{y} \psi_{1}^{\perp}, \psi_{2}\right\rangle \\
& =\left\langle\psi_{1}, \phi_{0}\right\rangle\left\langle a_{y} \phi_{0}, \psi_{2}\right\rangle+O\left(y^{2-s_{\Gamma}} \mathcal{S}_{2}\left(\psi_{2}\right) \cdot \mathcal{S}_{2}\left(\psi_{2}\right)\right)
\end{aligned}
$$

since $\mathcal{S}_{2}\left(\psi_{i}^{\perp}\right) \ll \mathcal{S}\left(\psi_{i}\right)$.

\section{Equidistribution of EXPANDing ClOSED hOROSPHERES With RESPECT TO THE Burger-RoBlin MEASURE}

Let $\Gamma<\mathrm{PSL}_{2}(\mathbb{C})$ be a geometrically finite Kleinian group with $\delta_{\Gamma}>1$. Assume that $\Gamma \backslash \Gamma N$ is closed. Let $\mu$ denote the Haar measure on $G=N A K$ given by

$$
d \mu\left(n_{x} a_{y} k\right)=y^{-3} d x d y d k,
$$

where $d k$ is the probability Haar measure on $K$. We normalize $\phi_{0}$ so that

$$
\int_{\Gamma \backslash \mathbb{H}^{3}} \phi_{0}(x, y)^{2} \frac{1}{y^{3}} d x d y=1 .
$$

By Corollary 4.13, we have

$$
\int_{n_{x} \in(N \cap \Gamma) \backslash N} \phi_{0}(x, y) d x=c_{\phi_{0}} y^{2-\delta_{\Gamma}}+d_{\phi_{0}} y^{\delta_{\Gamma}},
$$

where $c_{\phi_{0}}>0$ and $d_{\phi_{0}} \geq 0$.

In this section, we aim to prove the following theorem.

Theorem 6.1. For any $\psi \in C_{c}^{\infty}(\Gamma \backslash G)^{K}$,

$$
\int_{n_{x} \in(N \cap \Gamma) \backslash N} \psi\left(n_{x} a_{y}\right) d x=\left\langle\psi, \phi_{0}\right\rangle \cdot c_{\phi_{0}} \cdot y^{2-\delta_{\Gamma}}\left(1+O\left(y^{\frac{2}{7}\left(\delta_{\Gamma}-s_{\Gamma}\right)}\right)\right),
$$

where the implied constant depends only on the Sobolev norms of $\psi$, the volume of $N(\operatorname{supp}(\psi))$ and the volume of an open subset of $(N \cap \Gamma) \backslash N$ which properly covers $\Lambda_{N}(\Gamma)$.

Most of this section is devoted to a proof of Theorem 6.1.

Definition 6.2. For a given $\psi \in C^{\infty}(\Gamma \backslash G)^{K}$ and $\eta \in C_{c}((N \cap \Gamma) \backslash N)$, define the function $\mathrm{I}_{\eta}(\psi)$ on $G$ by

$$
\mathrm{I}_{\eta}(\psi)\left(a_{y}\right):=\int_{n_{x} \in(N \cap \Gamma) \backslash N} \psi\left(n_{x} a_{y}\right) \eta\left(n_{x}\right) d x .
$$

We denote by $N^{-}$the strictly lower triangular subgroup of $G$ :

$$
N^{-}:=\left\{\left(\begin{array}{ll}
1 & 0 \\
x & 1
\end{array}\right): x \in \mathbb{C}\right\} .
$$

The product map $N \times A \times N^{-} \times M \rightarrow G$ is a diffeomorphism at a neighborhood of $e$. Let $\nu$ be a smooth measure on $A N^{-} M$ such that $d n \otimes \nu\left(a n^{-} m\right)=d \mu$.

Fix a left-invariant Riemannian metric $d$ on $G$ and denote by $U_{\epsilon}$ the ball of radius $\epsilon$ about $e$ in $G$. 
Definition 6.3. - We fix a non-negative function $\eta \in C_{c}^{\infty}((N \cap \Gamma) \backslash N)$ with $\eta=1$ on a bounded open subset of $\mathcal{F}_{N}$ which properly covers $\Lambda_{N}(\Gamma)$.

- Fix $\epsilon_{0}>0$ so that for the $\epsilon_{0}$-neighborhood $U_{\epsilon_{0}}$ of $e$, the multiplication map

$$
\operatorname{supp}(\eta) \times\left(U_{\epsilon_{0}} \cap A N^{-} M\right) \rightarrow \operatorname{supp}(\eta)\left(U_{\epsilon_{0}} \cap A N^{-} M\right) \subset \Gamma \backslash G
$$

is a bijection onto its image.

- For each $\epsilon<\epsilon_{0}$, let $r_{\epsilon}$ be a non-negative smooth function in $A N^{-} M$ whose support is contained in

$$
W_{\epsilon}:=\left(U_{\epsilon} \cap A\right)\left(U_{\epsilon_{0}} \cap N^{-}\right)\left(U_{\epsilon_{0}} \cap M\right)
$$

and $\int_{W_{\epsilon}} r_{\epsilon} d \nu=1$.

- We define the following function $\rho_{\eta, \epsilon}$ on $\Gamma \backslash G$ which is 0 outside $\operatorname{supp}(\eta) U_{\epsilon_{0}}$ and for $g=n_{x} a n^{-} m \in \operatorname{supp}(\eta)\left(U_{\epsilon_{0}} \cap A N^{-} M\right)$,

$$
\rho_{\eta, \epsilon}(g):=\eta\left(n_{x}\right) \otimes r_{\epsilon}\left(a n^{-} m\right) .
$$

Recall from Proposition 4.6 that

$$
\phi_{0}^{N}\left(a_{y}\right)=\mathrm{I}_{\eta}\left(\phi_{0}\right)\left(a_{y}\right)+O\left(y^{\delta_{\Gamma}}\right) .
$$

Proposition 6.4. We have for all small $0<\epsilon \ll \epsilon_{0}$ and for all $0<y<1$,

$$
\phi_{0}^{N}\left(a_{y}\right)=\left\langle a_{y} \phi_{0}, \rho_{\eta, \epsilon}\right\rangle_{L^{2}(\Gamma \backslash G)}+O_{\eta}\left(\epsilon \cdot y^{2-\delta_{\Gamma}}\right)+O\left(y^{\delta_{\Gamma}}\right),
$$

where the first implied constant depends on the Lipschitz constant for $\eta$.

Proof. Let $h=a_{y_{0}} n_{x}^{-} m \in W_{\epsilon}$. Then for $n \in N$ and $y>0$, we have

$$
n h a_{y}=n a_{y y_{0}} n_{y x}^{-} m .
$$

As the product map $A \times N \times K \rightarrow G$ is a diffeomorphism and hence a bi-Lipschitz map at a neighborhood of $e$, there exists $\ell>0$ such that the $\epsilon$-neighborhood of $e$ in $G$ is contained in the product $A_{\ell \epsilon} N_{\ell \epsilon} K_{\ell \epsilon}$ of $\ell \epsilon$-neighborhoods for all small $\epsilon>0$.

Therefore we may write

$$
n_{y x}^{-}=a_{y_{1}} n_{x_{1}} k_{1} \in A_{\ell y \epsilon_{0}} N_{\ell y \epsilon_{0}} K
$$

and hence

$$
\begin{aligned}
n h a_{y} & =n a_{y y_{0} y_{1}} n_{x_{1}} k_{1} m \\
& =n\left(a_{y y_{0} y_{1}} n_{x_{1}} a_{y y_{0} y_{1}}^{-1}\right) a_{y y_{0} y_{1}} k_{1} m=n\left(n_{x_{1} y y_{0} y_{1}}\right) a_{y y_{0} y_{1}} k_{1} m .
\end{aligned}
$$

As $\phi_{0}$ is $K$-invariant and $d n$ is $N$-invariant,

$$
\begin{aligned}
\int_{N \cap \Gamma \backslash N} \phi_{0}\left(n h a_{y}\right) \cdot \eta(n) d n & =\int_{N \cap \Gamma \backslash N} \phi_{0}\left(n\left(n_{x_{1} y y_{0} y_{1}}\right) a_{y y_{0} y_{1}}\right) \cdot \eta(n) d n \\
& =\int_{N \cap \Gamma \backslash N} \phi_{0}\left(n a_{y y_{0} y_{1}}\right)(\eta(n)+O(\epsilon)) d n
\end{aligned}
$$


as $\eta(n)-\eta\left(n n^{\prime}\right)=O_{\eta}(\epsilon)$ for all $n \in N$ and $n^{\prime} \in N \cap U_{\epsilon}$. By Corollary 4.13, we deduce

$$
\begin{aligned}
\int_{N \cap \Gamma \backslash N} \phi_{0}\left(n h a_{y}\right) \cdot \eta(n) d n \\
\quad=\int_{N \cap \Gamma \backslash N} \phi_{0}\left(n a_{y y_{0} y_{1}}\right) \eta(n) d n+O_{\eta}\left(\epsilon \phi_{0}^{N}\left(a_{y y_{0} y_{1}}\right)\right) \\
=c_{\phi_{0}}\left(y y_{0} y_{1}\right)^{2-\delta_{\Gamma}}+d_{\phi_{0}}\left(y y_{0} y_{1}\right)^{\delta_{\Gamma}}+O_{\eta}\left(\epsilon y^{2-\delta_{\Gamma}}\right) \\
=c_{\phi_{0}} y^{2-\delta_{\Gamma}}\left(1+O\left(1-\left(y_{0} y_{1}\right)^{2-\delta_{\Gamma}}\right)\right)+O_{\eta}\left(\epsilon y^{2-\delta_{\Gamma}}\right)+O\left(y^{\delta_{\Gamma}}\right) \\
=c_{\phi_{0}} y^{2-\delta_{\Gamma}}(1+O(\epsilon))+O\left(y^{\delta_{\Gamma}}\right)
\end{aligned}
$$

as $\left|y_{0}-1\right|=O(\epsilon)$ and $\left|y_{1}-1\right|=O(y \epsilon)$.

As $\int r_{\epsilon} d \nu(h)=1$, we deduce

$$
\begin{aligned}
\left\langle a_{y} \phi_{0}, \rho_{\eta, \epsilon}\right\rangle & =\int_{W_{\epsilon}} r_{\epsilon}(h)\left(\int_{N \cap \Gamma \backslash N} \phi_{0}\left(n h a_{y}\right) \eta(n) d n\right) d \nu(h) \\
& =c_{\phi_{0}} y^{2-\delta_{\Gamma}}\left(1+O_{\eta}(\epsilon)\right)+O\left(y^{\delta_{\Gamma}}\right) \\
& =\phi_{0}^{N}\left(a_{y}\right)+O_{\eta}\left(\epsilon y^{2-\delta_{\Gamma}}\right)+O\left(y^{\delta_{\Gamma}}\right) .
\end{aligned}
$$

Lemma 6.5. For $\psi \in C_{c}^{\infty}(\Gamma \backslash G)^{K}$, there exists $\hat{\psi} \in C_{c}^{\infty}(\Gamma \backslash G)^{K}$ such that

(1) for all small $\epsilon>0$ and $h \in U_{\epsilon}$,

$$
|\psi(g)-\psi(g h)| \leq \epsilon \cdot \hat{\psi}(g) \quad \text { for all } g \in \Gamma \backslash G ;
$$

(2) $\mathcal{S}_{m}(\hat{\psi}) \ll \mathcal{S}_{5}(\psi)$ for any $m \in \mathbb{N}$, where the implied constant depends only on $\operatorname{supp}(\psi)$.

Proof. Fix $\epsilon_{0}>0$. Let $f_{0} \in C_{c}^{\infty}(\Gamma \backslash G)^{K}$ be such that $f_{0}(g)=1$ for all $g \in$ $\operatorname{supp}(\psi) U_{\epsilon_{0}}^{-1} K$ and $f_{0}(g)=0$ for all $g \in \Gamma \backslash G-\operatorname{supp}(\psi) U_{2 \epsilon_{0}}^{-1} K$.

Set $C_{\psi}:=\sup _{g \in \operatorname{supp}(\psi)} \sum_{i=1}^{6}\left|X_{i}(\psi)(g)\right|$. Then there exists a constant $c_{0} \geq 1$ such that for all $g \in \Gamma \backslash G$ and $h \in U_{\epsilon}$ for $\epsilon<\epsilon_{0}$,

$$
|\psi(g)-\psi(g h)| \leq \epsilon \cdot c_{0} C_{\psi} .
$$

Hence if we define $\hat{\psi} \in C_{c}^{\infty}(\Gamma \backslash G)^{K}$ by $\hat{\psi}(g)=c_{0} C_{\psi} f_{0}(g)$ for $g \in \Gamma \backslash G$, then (1) holds.

Now by the Sobolev imbedding theorem (cf. [1, Thm. 2.30]), we have

$$
C_{\psi} \leq \mathcal{S}_{5}(\psi)
$$

Since $\mathcal{S}_{m}(\hat{\psi}) \ll C_{\psi}$, this proves $(2)$.

Proposition 6.6. Let $\psi \in C^{\infty}(\Gamma \backslash G)^{K}$. Then for any $0<y<1$ and any small $\epsilon>0$,

$$
\left|\mathrm{I}_{\eta}(\psi)\left(a_{y}\right)-\left\langle a_{y} \psi, \rho_{\eta, \epsilon}\right\rangle\right| \ll(\epsilon+y) \cdot \mathrm{I}_{\eta}(\hat{\psi})\left(a_{y}\right) .
$$

Proof. If $a n^{-} m \in W_{\epsilon}=\left(U_{\epsilon} \cap A\right)\left(U_{\epsilon_{0}} \cap N^{-}\right)\left(U_{\epsilon_{0}} \cap M\right)$, then

$$
\left(a n^{-} m\right) a_{y}=a_{y} a\left(a_{y^{-1}} n^{-} a_{y}\right) m \in a_{y} W_{\epsilon}
$$

since $a_{y^{-1}}$ contracts $N^{-}$by conjugation as $0<y<1$. 
As $\psi$ is $M$-invariant, for any $h=a n^{-} m \in W_{\epsilon}$, there exists an $h^{\prime} \in\left(U_{\epsilon} \cap A\right)\left(U_{y \epsilon_{0}} \cap\right.$ $N^{-}$) such that

$$
\left|\psi\left(n a_{y}\right)-\psi\left(n h a_{y}\right)\right|=\left|\psi\left(n a_{y}\right)-\psi\left(n a_{y} h^{\prime}\right)\right| \ll \hat{\psi}\left(n a_{y}\right)(\epsilon+y) .
$$

Hence

$$
\left|\psi\left(n a_{y}\right)-\int_{h \in W_{\epsilon}} \psi\left(n h a_{y}\right) r_{\epsilon}(h) d \nu(h)\right| \ll \hat{\psi}\left(n a_{y}\right)(\epsilon+y) .
$$

Therefore

$$
\left|\mathrm{I}_{\eta}(\psi)\left(a_{y}\right)-\left\langle a_{y} \psi, \rho_{\epsilon}\right\rangle_{L^{2}(\Gamma \backslash G)}\right| \ll(\epsilon+y) \cdot \int_{(N \cap \Gamma) \backslash N} \hat{\psi}\left(n a_{y}\right) \eta(n) d n .
$$

Proof of Theorem 6.1. Recall that $\eta, \epsilon_{0}, \rho_{\eta, \epsilon}=\eta \otimes r_{\epsilon}$ are as in Definition 6.3. For simplicity, we set $\rho_{\epsilon}=\rho_{\eta, \epsilon}$. Noting that $r_{\epsilon}$ is essentially an $\epsilon$-approximation only in the $A$-direction, we obtain that $\mathcal{S}_{2}\left(\rho_{\epsilon}\right)=O_{\eta}\left(\epsilon^{-5 / 2}\right)$.

We may further assume that $\eta=1$ on $N(\operatorname{supp}(\psi))$ by Corollary 3.10 so that

$$
\int_{(N \cap \Gamma) \backslash N} \psi\left(n a_{y}\right) d n=\mathrm{I}_{\eta}(\psi)\left(a_{y}\right) .
$$

By Proposition 4.6, we also have

$$
\phi_{0}^{N}\left(a_{y}\right)=\mathrm{I}_{\eta}\left(\phi_{0}\right)\left(a_{y}\right)+O\left(y^{\delta_{\Gamma}}\right) .
$$

Set $p=5 / 2$. Fix $\ell \in \mathbb{N}$ so that

$$
\ell>\frac{\left(2-\delta_{\Gamma}\right)(p+1)}{\left(\delta_{\Gamma}-s_{\Gamma}\right)} .
$$

Setting $\psi_{0}(g):=\psi(g)$, we define for $1 \leq i \leq \ell$, inductively

$$
\psi_{i}(g):=\hat{\psi}_{i-1}(g),
$$

where $\hat{\psi}_{i-1}$ is given by Lemma 6.5 ,

Applying Proposition 6.6 to each $\psi_{i}$, we obtain for $0 \leq i \leq \ell-1$,

$$
\mathrm{I}_{\eta}\left(\psi_{i}\right)\left(a_{y}\right)=\left\langle a_{y} \psi_{i}, \rho_{\epsilon}\right\rangle+O\left((\epsilon+y) \mathrm{I}_{\eta}\left(\psi_{i+1}\right)\left(a_{y}\right)\right)
$$

and

$$
\mathrm{I}_{\eta}\left(\psi_{\ell}\right)\left(a_{y}\right)=\left\langle a_{y} \psi_{\ell}, \rho_{\epsilon}\right\rangle+O_{\eta}\left((\epsilon+y) \mathcal{S}_{2}\left(\psi_{\ell}\right)\right),
$$

where the implied constant in the $O_{\eta}$ notation depends on $\int \eta d n$.

Note that by Corollary 5.6. we have for each $1 \leq i \leq \ell$,

$$
\begin{aligned}
\left\langle a_{y} \psi_{i}, \rho_{\epsilon}\right\rangle & =\left\langle\psi_{i}, \phi_{0}\right\rangle\left\langle a_{y} \phi_{0}, \rho_{\epsilon}\right\rangle+O\left(y^{2-s_{\Gamma}} \mathcal{S}_{2}\left(\rho_{\epsilon}\right) \mathcal{S}_{2}\left(\psi_{i}\right)\right) \\
& =O\left(\left\langle a_{y} \phi_{0}, \rho_{\epsilon}\right\rangle \cdot\left\|\psi_{i}\right\|_{2}\right)+O\left(y^{2-s_{\Gamma}} \mathcal{S}_{2}\left(\rho_{\epsilon}\right) \mathcal{S}_{2}\left(\psi_{i}\right)\right) \\
& =O\left(\left\langle a_{y} \phi_{0}, \rho_{\epsilon}\right\rangle \cdot \mathcal{S}_{5}(\psi)\right)+O\left(y^{2-s_{\Gamma}} \epsilon^{-p} \mathcal{S}_{5}(\psi)\right) .
\end{aligned}
$$


Hence for any $y<\epsilon$,

$$
\begin{aligned}
& \mathrm{I}_{\eta}(\psi)\left(a_{y}\right)=\left\langle a_{y} \psi, \rho_{\epsilon}\right\rangle+\sum_{k=1}^{\ell-1} O\left(\left\langle a_{y} \psi_{k}, \rho_{\epsilon}\right\rangle(\epsilon+y)^{k}\right)+O_{\eta}\left(\mathcal{S}_{5}(\psi)(\epsilon+y)^{\ell}\right) \\
& =\left\langle a_{y} \psi, \rho_{\epsilon}\right\rangle+O\left(\left\langle a_{y} \phi_{0}, \rho_{\epsilon}\right\rangle \mathcal{S}_{5}(\psi)\right)+O\left(\epsilon \mathcal{S}_{5}(\psi) y^{2-s_{\Gamma}} \epsilon^{-p}\right)+O\left(\mathcal{S}_{5}(\psi) \epsilon^{\ell}\right) \\
& =\left\langle\psi, \phi_{0}\right\rangle \cdot\left\langle a_{y} \phi_{0}, \rho_{\epsilon}\right\rangle+O\left(\left\langle a_{y} \phi_{0}, \rho_{\epsilon}\right\rangle \epsilon\right)+O\left(y^{2-s_{\Gamma}} \epsilon^{-p}\right)+O\left(\epsilon^{\ell}\right) \\
& =\left\langle\psi, \phi_{0}\right\rangle \cdot \phi_{0}^{N}\left(a_{y}\right)+O\left(y^{\delta_{\Gamma}}\right)+O\left(\epsilon y^{2-\delta_{\Gamma}}\right)+O\left(y^{2-s_{\Gamma}} \epsilon^{-p}\right)+O\left(\epsilon^{\ell}\right)
\end{aligned}
$$

by Proposition 6.4, where the implied constants depend on the Sobolev norm $\mathcal{S}_{5}(\psi)$ and $\int \eta d n$.

Equating the two error terms $O\left(\epsilon y^{2-\delta_{\Gamma}}\right)$ and $O\left(y^{2-s_{\Gamma}} \epsilon^{-p}\right)$ gives the choice $\epsilon=$ $y^{\left(\delta_{\Gamma}-s_{\Gamma}\right) /(p+1)}$. By the condition on $\ell$, we then have $\epsilon^{\ell} \ll y^{2-\delta_{\Gamma}+\frac{2}{7}\left(\delta_{\Gamma}-s_{\Gamma}\right)}$. Hence we deduce:

$$
\int_{(N \cap \Gamma) \backslash N} \psi\left(n a_{y}\right) d n=\mathrm{I}_{\eta}(\psi)\left(a_{y}\right)=\left\langle\psi, \phi_{0}\right\rangle \cdot \phi_{0}^{N}\left(a_{y}\right) \cdot\left(1+O\left(y^{\frac{2}{7}\left(\delta_{\Gamma}-s_{\Gamma}\right)}\right) .\right.
$$

Note that the implied constant depends on the Sobolev norm $\mathcal{S}_{5}(\psi)$ of $\psi$ and the $L^{1}$-norm $\int \eta d n$, which in turn depends only on the volumes $N(\operatorname{supp}(\psi))$ and any open subset of $(N \cap \Gamma) \backslash N$ which properly covers $\Lambda_{N}(\Gamma)$.

Burger-Roblin measure $\hat{\mu}$ : In identifying $\partial_{\infty}\left(\mathbb{H}^{3}\right)$ with $K / M$, we may define the following measure $\hat{\mu}$ on $\mathrm{T}^{1}\left(\mathbb{H}^{3}\right)=G / M$ : for $\psi \in C_{c}(G / M)$,

$$
\hat{\mu}(\psi)=\int_{k \in K} \int_{a_{y} n_{x} \in A N} \psi\left(k a_{y} n_{x}\right) y^{\delta-1} d y d x d \nu_{o}(k),
$$

where we consider the Patterson-Sullivan measure $\nu_{o}$ in section 4 as a measure on $K$ via the projection $K \rightarrow K / M$ : for $f \in C(K)$,

$$
\nu_{o}(f)=\int_{k \in K / M} \int_{m \in M} f(k m) d m d \nu_{o}(k)
$$

for the probability invariant measure $d m$ on $M$.

By the conformal property of $\nu_{o}$, the measure $\hat{\mu}$ is left $\Gamma$-invariant and hence induces a Radon measure on $T^{1}\left(\Gamma \backslash \mathbb{H}^{3}\right)$ via the canonical projection.

Lemma 6.7. For a $K$-invariant function $\psi \in C_{c}(G)$, we have

$$
\hat{\mu}(\psi)=\left\langle\psi, \phi_{0}\right\rangle .
$$

Proof. It is easy to compute that for the base point $o=(0,0,1) \in \mathbb{H}^{3}$,

$$
\beta_{\infty}\left(a_{y} n_{x} o, o\right)=-\log y
$$


for any $0<y \leq 1$ and $x \in \mathbb{C}$. We note that $d g=y^{-1} d y d x d k$ for $g=a_{y} n_{x} k$ is a Haar measure on $G$. Therefore, using that $\psi$ is $K$-invariant,

$$
\begin{aligned}
& \hat{\mu}(\psi)=\int_{K} \int_{A N} \int_{k_{0} \in K} \psi\left(k a_{y} n_{x} k_{0}\right) d\left(k_{0}\right) y^{\delta-1} d y d x d \nu_{o}(k) \\
& =\int_{g \in G} \int_{K} \psi(k g) e^{-\delta \beta_{\infty}(g o, o)} d \nu_{o}(k) d g \\
& =\int_{G} \psi(g) \int_{k \in K / M} e^{-\delta \beta_{\infty}\left(k^{-1} g o, o\right)} d \nu_{o}(k) d g \\
& =\int_{G} \psi(g) \int_{K / M} e^{-\delta \beta_{k(\infty)}(g o, o)} d \nu_{o}(k) d g=\left\langle\psi, \phi_{0}\right\rangle
\end{aligned}
$$

as $\phi_{0}(g)=\int_{K / M} e^{-\delta \beta_{k(\infty)}(g o, o)} d \nu_{o}(k)$.

Generalizing Burger's result [7, Roblin [38, Thm. 6.4] proved:

Theorem 6.9. The measure $\hat{\mu}$ is, up to a constant multiple, the unique Radon measure on $\mathrm{T}^{1}\left(\Gamma \backslash \mathbb{H}^{3}\right)$ invariant for the horospherical foliations whose support is $\hat{\Omega}_{\Gamma}$ and which gives zero measure to closed horospheres.

We call the measure $\hat{\mu}$ the Burger-Roblin measure.

In the appendix, the following theorem is deduced from Theorem 6.1

Theorem 6.10. For $\psi \in C_{c}\left(\mathrm{~T}^{1}\left(\Gamma \backslash \mathbb{H}^{3}\right)\right)$,

$$
\int_{n_{x} \in(N \cap \Gamma) \backslash N} \psi\left(n_{x} a_{y}\right) d x \sim c_{\phi_{0}} \cdot \hat{\mu}(\psi) \cdot y^{2-\delta_{\Gamma}} \quad \text { as } y \rightarrow 0 .
$$

\section{Orbital COUnting for a KLEINian group}

Let $\iota: G=\mathrm{PSL}_{2}(\mathbb{C}) \rightarrow \mathrm{SO}_{F}(\mathbb{R})$ be a real linear representation, where $F$ is a real quadratic form in 4 variables of signature $(3,1)$. Let $\Gamma<G$ be a geometrically finite torsion-free discrete subgroup with $\delta_{\Gamma}>1$. Let $v_{0} \in \mathbb{R}^{4}$ be a non-zero (row) vector with $F\left(v_{0}\right)=0$ such that the orbit $v_{0} \Gamma$ is discrete in $\mathbb{R}^{4}$.

Since the orthogonal group $\mathrm{O}_{F}(\mathbb{R})$ acts transitively on the set of non-zero vectors of the cone $F=0$, there exists $g_{0}^{t} \in \mathrm{O}_{F}(\mathbb{R})$ such that the stabilizer of $g_{0}^{t} v_{0}^{t}$ is equal to $N^{-} M$, where $N^{-}$is the strictly lower triangular subgroup. In fact, $g_{0}^{t} v_{0}^{t}$ is unique up to homothety. Set

$$
\Gamma_{0}=g_{0}^{-1} \Gamma g_{0} .
$$

As $v_{0} \Gamma$ is discrete, it follows that $\Gamma_{0} \backslash \Gamma_{0} N M$ is closed. Hence by Lemma 3.4, the orbit $\Gamma \backslash \Gamma g_{0} N$ is closed; equivalently $\Gamma_{0} \backslash \Gamma_{0} N$ is closed.

Denote by $\phi_{0} \in L^{2}\left(\Gamma_{0} \backslash \mathbb{H}^{3}\right)$ the unique positive eigenfunction of $\Delta$ with eigenvalue $\delta_{\Gamma}\left(2-\delta_{\Gamma}\right)$ and of unit $L^{2}$-norm $\int_{\Gamma_{0} \backslash \mathbb{H}^{3}} \phi_{0}(x, y)^{2} y^{-3} d x d y=1$. By Corollary 4.13, we have

$$
\phi_{0}^{N}\left(a_{y}\right)=c_{\phi_{0}} y^{2-\delta_{\Gamma}}+d_{\phi_{0}} y^{\delta_{\Gamma}},
$$

where $c_{\phi_{0}}>0$ and $d_{\phi_{0}} \geq 0$. Recall the Patterson-Sullivan measure $\nu_{o}$ on $K$, which is normalized so that

$$
\phi_{0}(x, y)=\int_{u \in \Lambda(\Gamma) \backslash\{\infty\}}\left(\frac{\left(\|u\|^{2}+1\right) y}{\|x-u\|^{2}+y^{2}}\right)^{\delta_{\Gamma}} d \nu_{o}(u) .
$$


Theorem 7.1. For any norm $\|\cdot\|$ on $\mathbb{R}^{4}$, we have, as $T \rightarrow \infty$,

$$
\#\left\{v \in v_{0} \Gamma:\|v\|<T\right\} \sim \delta_{\Gamma}^{-1} \cdot c_{\phi_{0}} \cdot\left(\int_{k \in K}\left\|v_{0}\left(g_{0} k^{-1} g_{0}^{-1}\right)\right\|^{-\delta_{\Gamma}} d \nu_{o}(k)\right) \cdot T^{\delta_{\Gamma}} .
$$

If $\|\cdot\|$ is $g_{0} K g_{0}^{-1}$-invariant, then there exists $\epsilon>0$ such that

$$
\#\left\{v \in v_{0} \Gamma:\|v\|<T\right\}=\delta_{\Gamma}^{-1} \cdot \phi_{0}(e) \cdot c_{\phi_{0}} \cdot\left\|v_{0}\right\|^{-\delta_{\Gamma}} \cdot T^{\delta_{\Gamma}}\left(1+O\left(T^{-\epsilon}\right)\right),
$$

where $\epsilon$ depends only on the spectral gap $\delta_{\Gamma}-s_{\Gamma}$ and the implied constant depends only on $\Lambda_{N}(\Gamma)$.

By replacing $\Gamma$ with $\Gamma_{0}=g_{0}^{-1} \Gamma g_{0}$, we may assume henceforth that $g_{0}=e$, and thus the stabilizer of $v_{0}$ in $G$ is $N M$. By Lemma 3.4, the stabilizer of $v_{0}$ in $\Gamma$ is simply $\Gamma \cap N$.

Note that $N^{-} M$ fixes $v_{0}^{t}$, and that the highest weight $\beta$ of the (irreducible) representation of $\iota$ is given by $\beta\left(a_{y}\right)=y^{-1}$. It follows that $\iota\left(a_{y}\right) v_{0}^{t}=y^{-1} v_{0}^{t}$, and hence $v_{0} a_{y}=y^{-1} v_{0}$.

Set

$$
B_{T}:=\left\{v \in v_{0} G:\|v\|<T\right\} .
$$

Define the following function on $\Gamma \backslash G$ :

$$
F_{T}(g):=\sum_{\gamma \in(N \cap \Gamma) \backslash \Gamma} \chi_{B_{T}}\left(v_{0} \gamma g\right)
$$

Since $v_{0} \Gamma$ is discrete, $F_{T}(g)$ is well defined and

$$
F_{T}(e)=\#\left\{v \in v_{0} \Gamma:\|v\|<T\right\} .
$$

We use the notation: for $\psi \in C_{c}(\Gamma \backslash G)$,

$$
\psi^{N}\left(a_{y}\right):=\int_{(N \cap \Gamma) \backslash N} \psi\left(n a_{y}\right) d n .
$$

Lemma 7.2. For any $\psi \in C_{c}(\Gamma \backslash G)$ and $T>0$,

$$
\left\langle F_{T}, \psi\right\rangle=\int_{k \in M \backslash K} \int_{y>T^{-1}\left\|v_{0} k\right\|} \psi_{k}^{N}\left(a_{y}\right) y^{-3} d y d k,
$$

where $\psi_{k}(g)=\int_{m \in M} \psi(g m k) d m$. 
Proof. Observe:

$$
\begin{aligned}
\left\langle F_{T}, \psi\right\rangle & =\int_{\Gamma \backslash G} \sum_{\gamma \in(N \cap \Gamma) \backslash \Gamma} \chi_{B_{T}}\left(v_{0} \gamma g\right) \psi(g) d g \\
& =\int_{g \in(N \cap \Gamma) \backslash G} \chi_{B_{T}}\left(v_{0} g\right) \psi(g) d g \\
& =\int_{k \in M \backslash K} \int_{y:\left\|v_{0} a_{y} k\right\|<T} \int_{n_{x} m \in(N \cap \Gamma) \backslash N M} \psi\left(n_{x} m a_{y} k\right) y^{-3} d x d m d y d k \\
& =\int_{k \in M \backslash K} \int_{y>T^{-1}\left\|v_{0} k\right\|}\left(\int_{n_{x} \in(N \cap \Gamma) \backslash N} \int_{m \in M} \psi\left(n_{x} a_{y} m k\right) d m d x\right) y^{-3} d y d k \\
& =\int_{k \in M \backslash K} \int_{y>T^{-1}\left\|v_{0} k\right\|}\left(\int_{n_{x} \in(N \cap \Gamma) \backslash N} \psi_{k}\left(n_{x} a_{y}\right) d x\right) y^{-3} d y d k \\
& =\int_{k \in M \backslash K} \int_{y>T^{-1}\left\|v_{0} k\right\|} \psi_{k}^{N}\left(a_{y}\right) y^{-3} d y d k .
\end{aligned}
$$

Define a function $\xi_{v_{0}}: K \rightarrow \mathbb{R}$ by

$$
\xi_{v_{0}}(k)=\left\|v_{0} k\right\|^{-\delta_{\Gamma}} .
$$

For $\psi \in C_{c}(\Gamma \backslash G)$, the convolution $\xi_{v_{0}} * \psi$ is then given by

$$
\xi_{v_{0}} * \psi(g)=\int_{k \in K} \psi(g k)\left\|v_{0} k\right\|^{-\delta_{\Gamma}} d k .
$$

Corollary 7.3. For any $\psi \in C_{c}(\Gamma \backslash G)$, we have, as $T \rightarrow \infty$,

$$
\left\langle F_{T}, \psi\right\rangle \sim \delta_{\Gamma}^{-1} \cdot c_{\phi_{0}} \cdot T^{\delta_{\Gamma}} \cdot \hat{\mu}\left(\xi_{v_{0}} * \psi\right) .
$$

For $\psi \in C_{c}^{\infty}(\Gamma \backslash G)^{K}$ and $\|\cdot\| K$-invariant,

$$
\left\langle F_{T}, \psi\right\rangle=\left\langle\psi, \phi_{0}\right\rangle \cdot \delta_{\Gamma}^{-1} \cdot c_{\phi_{0}} \cdot T^{\delta_{\Gamma}} \cdot\left\|v_{0}\right\|^{-\delta_{\Gamma}}\left(1+O\left(T^{-\frac{2}{7}\left(\delta_{\Gamma}-s_{\Gamma}\right)}\right)\right),
$$

where $s_{\Gamma}$ is as in Definition 5.5 and the implied constant depends only on the Sobolev norm of $\psi, \Lambda_{N}(\Gamma)$ and $N(\operatorname{supp}(\psi))$.

Proof. Note that for any $\psi \in C_{c}(\Gamma \backslash G)$ and $k \in K$, the function $\psi_{k}$, defined in Lemma 7.2, is $M$-invariant. Applying Theorem 6.10 to $\psi_{k}$, we obtain that as $y \rightarrow 0$,

$$
\int_{n_{x} \in(N \cap \Gamma) \backslash N} \psi_{k}\left(n_{x} a_{y}\right) d x \sim c_{\phi_{0}} \cdot y^{2-\delta_{\Gamma}} \cdot \hat{\mu}\left(\psi_{k}\right) .
$$

Hence by applying Lemma 7.2, inserting the definition of $\psi_{k}$, and evaluating the $y$-integral, we get

$$
\begin{aligned}
\left\langle F_{T}, \psi\right\rangle & \sim c_{\phi_{0}} \cdot \int_{M \backslash K} \int_{y>T^{-1}\left\|v_{0} k\right\|} y^{-1-\delta_{\Gamma}} \hat{\mu}\left(\psi_{k}\right) d y d k \\
& =\delta_{\Gamma}^{-1} \cdot c_{\phi_{0}} \cdot T^{\delta_{\Gamma}} \int_{M \backslash K} \int_{M} \hat{\mu}(\psi(g m k)) \cdot\left\|v_{0} k\right\|^{-\delta_{\Gamma}} d m d k \\
& =\delta_{\Gamma}^{-1} \cdot c_{\phi_{0}} \cdot T^{\delta_{\Gamma}} \cdot \hat{\mu}\left(\xi_{v_{0}} * \psi\right),
\end{aligned}
$$

proving the first claim. 
Now suppose that both $\psi$ and the norm $\|\cdot\|$ are $K$-invariant.

As $\psi_{k}=\psi$, by Theorem 6.1, we can replace (7.4) by an asymptotic formula with power savings error term:

$$
\int_{n_{x} \in(N \cap \Gamma) \backslash N} \psi\left(n_{x} a_{y}\right) d x=c_{\phi_{0}} y^{2-\delta_{\Gamma}}\left\langle\psi, \phi_{0}\right\rangle\left(1+O\left(y^{\frac{2}{7}\left(\delta_{\Gamma}-s_{\Gamma}\right)}\right)\right),
$$

and the implied constant depends on the Sobolev norm of $\psi$ and $\Lambda_{N}(\Gamma)$.

On the other hand,

$$
\xi_{v_{0}} * \psi=\left\|v_{0}\right\|^{-\delta_{\Gamma}} \cdot \psi,
$$

and hence

$$
\hat{\mu}\left(\xi_{v_{0}} * \psi\right)=\left\|v_{0}\right\|^{-\delta_{\Gamma}} \cdot\left\langle\psi, \phi_{0}\right\rangle
$$

Therefore

$$
\begin{aligned}
\left\langle F_{T}, \psi\right\rangle & =c_{\phi_{0}} \int_{y>T^{-1}\left\|v_{0}\right\|} y^{-1-\delta_{\Gamma}}\left\langle\psi, \phi_{0}\right\rangle\left(1+O\left(y^{\frac{2}{7}\left(\delta_{\Gamma}-s_{\Gamma}\right)}\right)\right) d y \\
& =\delta_{\Gamma}^{-1} \cdot c_{\phi_{0}} \cdot T^{\delta_{\Gamma}} \cdot\left\|v_{0}\right\|^{-\delta_{\Gamma}}\left(1+O\left(T^{-\frac{2}{7}\left(\delta_{\Gamma}-s_{\Gamma}\right)}\right)\right),
\end{aligned}
$$

where the implied constant depends only on the Sobolev norm of $\psi$ and the set $\Lambda_{N}(\Gamma)$.

Theorem 7.5. As $T \rightarrow \infty$,

$$
F_{T}(e) \sim \delta_{\Gamma}^{-1} \cdot c_{\phi_{0}} \cdot\left(\int_{K}\left\|v_{0} k^{-1}\right\|^{-\delta_{\Gamma}} d \nu_{o}(k)\right) \cdot T^{\delta_{\Gamma}} .
$$

If $\|\cdot\|$ is $K$-invariant, then for some $\epsilon>0$ (depending only on the spectral gap $\left.\delta_{\Gamma}-s_{\Gamma}\right)$,

$$
F_{T}(e)=\delta_{\Gamma}^{-1} \cdot \phi_{0}(e) \cdot c_{\phi_{0}} \cdot\left\|v_{0}\right\|^{-\delta_{\Gamma}} \cdot T^{\delta_{\Gamma}}\left(1+O\left(T^{-\epsilon}\right)\right),
$$

where the implied constant depends only on $\Lambda_{N}(\Gamma)$.

Proof. For all small $\epsilon>0$, we choose a symmetric $\epsilon$-neighborhood $U_{\epsilon}$ of $e$ in $G$, which injects to $\Gamma \backslash G$, such that for all $T \gg 1$ and all $0<\epsilon \ll 1$,

$$
B_{T} U_{\epsilon} \subset B_{(1+\epsilon) T} \quad \text { and } \quad B_{(1-\epsilon) T} \subset \bigcap_{u \in U_{\epsilon}} B_{T} u .
$$

For $\epsilon>0$, let $\phi_{\epsilon} \in C_{c}^{\infty}(G)$ denote a non-negative function supported on $U_{\epsilon}$ and with $\int_{G} \phi_{\epsilon} d g=1$. We lift $\phi_{\epsilon}$ to $\Gamma \backslash G$ by averaging over $\Gamma$ :

$$
\Phi_{\epsilon}(\Gamma g)=\sum_{\gamma \in \Gamma} \phi_{\epsilon}(\gamma g) .
$$

Then

$$
\left\langle F_{(1-\epsilon) T}, \Phi_{\epsilon}\right\rangle \leq F_{T}(e) \leq\left\langle F_{(1+\epsilon) T}, \Phi_{\epsilon}\right\rangle .
$$

Note that

$$
\left\langle F_{(1 \pm \epsilon) T}, \Phi_{\epsilon}\right\rangle \sim \delta_{\Gamma}^{-1} \cdot c_{\phi_{0}} \cdot(T(1 \pm \epsilon))^{\delta_{\Gamma}} \cdot \hat{\mu}\left(\xi_{v_{0}} * \Phi_{\epsilon}\right) .
$$

Considering the function $R_{v_{0}}: G \rightarrow \mathbb{R}$ given by

$$
R_{v_{0}}(g):=y^{\delta_{\Gamma}} \xi_{v_{0}}\left(k_{0}\right)
$$


for $g=a_{y} n_{x} k_{0} \in A N K$, we have

$$
\begin{aligned}
\hat{\mu}\left(\xi_{v_{0}} * \Phi_{\epsilon}\right) & =\int_{g \in U_{\epsilon}} \int_{k_{0} \in K} \phi_{\epsilon}\left(g k_{0}\right) \xi_{v_{0}}\left(k_{0}\right) d\left(k_{0}\right) d \hat{\mu}(g) \\
& =\int_{k a_{y} n_{x} \in K A N \cap U_{\epsilon}} \int_{k_{0} \in K} \phi_{\epsilon}\left(k a_{y} n_{x} k_{0}\right) \xi_{v_{0}}\left(k_{0}\right) y^{\delta-1} d\left(k_{0}\right) d y d x d \nu_{o}(k) \\
& =\int_{k \in K} \int_{g \in G} \phi_{\epsilon}(k g) R(g) d g d \nu_{o}(k) \\
& =\int_{g \in U_{\epsilon}} \phi_{\epsilon}(g) \int_{k \in K} R\left(k^{-1} g\right) d \nu_{o}(k) d g \\
& =\int_{k \in K} R\left(k^{-1}\right) d \nu_{o}(k)+O(\epsilon) \\
& =\int_{k \in K / M}\left\|v_{0} k^{-1}\right\|^{-\delta_{\Gamma}} d \nu_{o}(k)+O(\epsilon)
\end{aligned}
$$

as $\int_{G} \phi_{\epsilon} d g=1$, where the implied constant depends only on the Lipschitz constant for $R$.

As $\epsilon>0$ is arbitrary, we deduce that

$$
F_{T}(e) \sim \delta_{\Gamma}^{-1} \cdot c_{\phi_{0}} \cdot \int_{k \in K}\left\|v_{0} k^{-1}\right\|^{-\delta_{\Gamma}} d \nu_{o}(k) \cdot T^{\delta_{\Gamma}} .
$$

If $\|\cdot\|$ is $K$-invariant, we may take both $U_{\epsilon}$ and $\phi_{\epsilon}$ to be $K$-invariant. Hence by Corollary [7.3, we may replace (7.7) by

$$
\left\langle F_{(1 \pm \epsilon) T}, \Phi_{\epsilon}\right\rangle=\delta_{\Gamma}^{-1} \cdot c_{\phi_{0}} \cdot\left\|v_{0}\right\|^{-\delta_{\Gamma}} \cdot(T(1 \pm \epsilon))^{\delta_{\Gamma}} \cdot\left\langle\phi_{0}, \Phi_{\epsilon}\right\rangle \cdot\left(1+O\left(T^{-\frac{2}{7}\left(\delta_{\Gamma}-s_{\Gamma}\right)}\right)\right),
$$

where the implied constant depends on $\mathcal{S}_{5}\left(\Phi_{\epsilon}\right)=\mathcal{S}_{5}\left(\phi_{\epsilon}\right)$ and the sets $\Lambda_{N}(\Gamma)$ and $N\left(U_{\epsilon}\right):=\left\{[n] \in(N \cap \Gamma) \backslash N: \Gamma n A \cap U_{\epsilon} \neq \emptyset\right\}$.

Since

$$
\left\langle\phi_{0}, \Phi_{\epsilon}\right\rangle=\phi_{0}(e)+O(\epsilon)
$$

we have

$$
\left\langle F_{(1 \pm \epsilon) T}, \Phi_{\epsilon}\right\rangle=\delta_{\Gamma}^{-1} \cdot c_{\phi_{0}} \cdot\left\|v_{0}\right\|^{-\delta_{\Gamma}} \cdot T^{\delta_{\Gamma}} \cdot \phi_{0}(e)+O\left(\epsilon T^{\delta_{\Gamma}}\right)+O\left(\epsilon^{-q} T^{\delta_{\Gamma}-\frac{2}{7}\left(\delta_{\Gamma}-s_{\Gamma}\right)}\right)
$$

for some $q>0$ depending on $\mathcal{S}_{5}\left(\phi_{\epsilon}\right)$. Therefore by setting $\epsilon^{1+q}=T^{-\frac{2}{7}\left(\delta_{\Gamma}-s_{\Gamma}\right)}$,

$$
F_{T}(e)=\delta_{\Gamma}^{-1} \cdot c_{\phi_{0}} \cdot\left\|v_{0}\right\|^{-\delta_{\Gamma}} \cdot T^{\delta_{\Gamma}} \cdot \phi_{0}(e) \cdot\left(1+O\left(T^{-\epsilon^{\prime}}\right)\right)
$$

for $\epsilon^{\prime}=\frac{2}{7(1+q)}\left(\delta_{\Gamma}-s_{\Gamma}\right)$, where the implied constant depends only on $\Lambda_{N}(\Gamma)$.

\section{The Selberg sieve and Circles of Prime curvature}

8.1. Selberg's sieve. We first recall the Selberg upper bound sieve. Let A denote the finite sequence of real non-negative numbers $\mathrm{A}=\left\{a_{n}\right\}$, and let $P$ be a finite product of distinct primes. We are interested in an upper bound for the quantity

$$
S(\mathrm{~A}, P):=\sum_{(n, P)=1} a_{n}
$$

To estimate $S(\mathrm{~A}, P)$ we need to know how A is distributed along certain arithmetic progressions. For $d \nmid P$, define

$$
\mathrm{A}_{d}:=\left\{a_{n} \in \mathrm{A}: n \equiv 0(d)\right\}
$$


and set $\left|\mathrm{A}_{d}\right|:=\sum_{n \equiv 0(d)} a_{n}$. We record

Theorem 8.1 ([19, Theorem 6.4]). Suppose that there exists a finite set $S$ of primes such that $P$ has no prime factor in $S$. Suppose that there exist $\mathcal{X}>1$ and a function $g$ on square-free integers with $0<g(p)<1$ for $p \mid P$ and $g$ is multiplicative outside $S$ (i.e., $g\left(d_{1} d_{2}\right)=g\left(d_{1}\right) g\left(d_{2}\right)$ if $d_{1}$ and $d_{2}$ are square-free integers with no factors in $S)$ such that for all $d \nmid P$ square-free,

$$
\left|\mathrm{A}_{d}\right|=g(d) \mathcal{X}+r_{d}(\mathrm{~A}) .
$$

Let $h$ be the multiplicative function on square-free integers (outside $S$ ) given by $h(p)=\frac{g(p)}{1-g(p)}$. Then for any $D>1$, we have that

$$
S(\mathrm{~A}, P) \leq \mathcal{X}\left(\sum_{d<\sqrt{D}, d \mid P} h(d)\right)^{-1}+\sum_{d<D, d \mid P} \tau_{3}(d) \cdot\left|r_{d}(\mathrm{~A})\right|,
$$

where $\tau_{3}(d)$ denotes the number of representations of $d$ as the product of three natural numbers.

8.2. Executing the sieve. Recall from section 2 that $Q$ denotes the Descartes quadratic form and $\mathcal{A}$ denotes the Apollonian group in $\mathrm{O}_{Q}(\mathbb{Z})$. Fix a primitive integral Apollonian packing $\mathcal{P}$ with its root quadruple $\xi \in \mathbb{Z}^{4}$. Then $\mathcal{P}$ is either bounded or given by the circle packing in Figure 3 .

To execute the sieve, it is important to work with a simply connected group. Hence we will set $\Gamma_{\mathcal{A}}$ to be the preimage of $\mathrm{SO}_{Q}(\mathbb{R})^{\circ} \cap \mathcal{A}$ in the spin double cover $\operatorname{Spin}_{Q}(\mathbb{R})$ of $\mathrm{SO}_{Q}(\mathbb{R})$. Recall that $\alpha$ denotes the Hausdorff dimension of the residual set of the packing $\mathcal{P}$. As shown in section 2.5, $\alpha$ is equal to $\delta_{\Gamma_{\mathcal{A}}}$, the Hausdorff dimension of the limit set of $\Gamma_{\mathcal{A}}$. As $\operatorname{Spin}_{Q}(\mathbb{R})$ is isomorphic to $\mathrm{SL}_{2}(\mathbb{C})$, we have a real linear representation $\iota: \mathrm{SL}_{2}(\mathbb{C}) \rightarrow \mathrm{SO}_{Q}(\mathbb{R})$ which factors through the quotient map $\mathrm{SL}_{2}(\mathbb{C}) \rightarrow \mathrm{PSL}_{2}(\mathbb{C})$. By setting $\Gamma$ to be the preimage of $\Gamma_{\mathcal{A}}$ under $\iota$, the counting results in the previous section are all valid for $\Gamma$.

As before, $B_{T}$ denotes the ball in the cone:

$$
B_{T}:=\left\{v \in \mathbb{R}^{4}: Q\left(v^{t}\right)=0,\|v\|<T\right\} .
$$

Since we are only looking for an upper bound, we may assume $\|\cdot\|$ is $g_{0} K g_{0}^{-1}$ invariant, where $g_{0} \in \mathrm{O}_{Q}(\mathbb{R})$ is such that the stabilizer of $g_{0}^{t} v_{0}^{t}$ is equal to $N^{-} M$. We fix a small $\epsilon>0$ and let $\phi_{\epsilon} \in C_{c}^{\infty}\left(\mathbb{H}^{3}\right)=C_{c}^{\infty}(G)^{K}$ be as in the proof of Theorem 7.5 with $G=\mathrm{SL}_{2}(\mathbb{C})$ and $K=\mathrm{SU}(2)$.

Definition 8.3 (Weight). We define the smoothed weight to each $\gamma \in \Gamma$,

$$
w_{T}(\gamma):=\int_{G / K} \chi_{B_{T}}(\xi \gamma g) \phi_{\epsilon}(g) d \mu(g) .
$$

Let $f$ be a primitive integral polynomial in 4 variables. Consider the sequence $\mathrm{A}(T)=\left\{a_{n}(T)\right\}$, where

$$
a_{n}(T):=\sum_{\substack{\gamma \in \operatorname{Stab}_{\Gamma}(\xi) \backslash \Gamma \\ f(\xi \gamma)=n}} w_{T}(\gamma)
$$

Clearly,

$$
|\mathrm{A}(T)|=\sum_{n} a_{n}(T)=\sum_{\gamma \in \operatorname{Stab}_{\Gamma}(\xi) \backslash \Gamma} w_{T}(\gamma)
$$


and

$$
\left|\mathrm{A}_{d}(T)\right|=\sum_{n \equiv 0(d)} a_{n}(T)=\sum_{\substack{\gamma \in \Gamma \\ f(\xi \gamma) \equiv 0(d)}} w_{T}(\gamma)
$$

For any subgroup $\Gamma_{0}$ of $\Gamma$ with

$$
\operatorname{Stab}_{\Gamma}(\xi)=\operatorname{Stab}_{\Gamma_{0}}(\xi)
$$

we define

$$
F_{T}^{\Gamma_{0}}(g):=\sum_{\gamma \in \operatorname{Stab}_{\Gamma}(\xi) \backslash \Gamma_{0}} \chi_{B_{T}}(\xi \gamma g)
$$

and for each element $\gamma_{1} \in \Gamma$, we also define a function on $\Gamma_{0} \backslash \mathbb{H}^{3}$ by

$$
\Phi_{\epsilon, \gamma_{1}}^{\Gamma_{0}}(g):=\sum_{\gamma \in \Gamma_{0}} \phi_{\epsilon}\left(\gamma_{1}^{-1} \gamma g\right)
$$

which is an $\epsilon$-approximation to the identity about $\left[\gamma_{1}^{-1}\right]$ in $\Gamma_{0} \backslash \mathbb{H}^{3}$. The function $\Phi_{\epsilon, e}^{\Gamma_{0}}$ is simply the lift of $\phi_{\epsilon}$ to $\Gamma_{0} \backslash \mathbb{H}^{3}$ and will be denoted by $\Phi_{\epsilon}^{\Gamma_{0}}$.

For $d \in \mathbb{Z}$, let $\Gamma_{\xi}(d)$ be the subgroup of $\Gamma$ which stabilizes $\xi \bmod d$, i.e.,

$$
\Gamma_{\xi}(d):=\{\gamma \in \Gamma: \xi \gamma \equiv \xi(d)\} .
$$

Note that

$$
\operatorname{Stab}_{\Gamma}(\xi)=\operatorname{Stab}_{\Gamma_{\xi}(d)}(\xi) .
$$

Lemma 8.5. (1) $|\mathrm{A}(T)|=\left\langle F_{T}^{\Gamma}, \Phi_{\epsilon}^{\Gamma}\right\rangle_{L^{2}\left(\Gamma \backslash \mathbb{H}^{3}\right)}$.

(2) For any integer $d$,

$$
\left|\mathrm{A}_{d}(T)\right|=\sum_{\substack{\gamma_{1} \in \Gamma_{\xi}^{(d) \backslash \Gamma} \\ f\left(\xi \gamma_{1}\right) \equiv 0(d)}}\left\langle F_{T}^{\Gamma_{\xi}^{(d)}}, \Phi_{\epsilon, \gamma_{1}}^{\Gamma_{\xi}(d)}\right\rangle_{L^{2}\left(\Gamma_{\xi}(d) \backslash \mathbb{H}^{3}\right)} .
$$

Proof. We have

$$
\begin{aligned}
|\mathrm{A}(T)| & =\sum_{\gamma \in \operatorname{Stab}_{\Gamma}(\xi) \backslash \Gamma} w_{T}(\gamma) \\
& =\sum_{\gamma \in \operatorname{Stab}_{\Gamma}(\xi) \backslash \Gamma} \int_{G / K} \chi_{B_{T}}(\xi \gamma g) \phi_{\epsilon}(g) d \mu(g) \\
& =\int_{G / K} F_{T}^{\Gamma}(g) \phi_{\epsilon}(g) d \mu(g) \\
& =\int_{\Gamma \backslash G / K} F_{T}^{\Gamma}(g) \Phi_{\epsilon}^{\Gamma}(g) d \mu(g) \\
& =\left\langle F_{T}^{\Gamma}, \Phi_{\epsilon}^{\Gamma}\right\rangle_{L^{2}\left(\Gamma \backslash \mathbb{H}^{3}\right)} .
\end{aligned}
$$

Expand (8.4) as

$$
\left|\mathrm{A}_{d}(T)\right|=\sum_{\substack{\gamma_{1} \in \Gamma_{\xi}(d) \backslash \Gamma \\ f\left(\xi \gamma_{1}\right) \equiv 0(d)}} \sum_{\gamma \in \operatorname{Stab}_{\Gamma}(\xi) \backslash \Gamma_{\xi}(d)} w_{T}\left(\gamma \gamma_{1}\right) .
$$


The inner sum is

$$
\begin{aligned}
\sum_{\gamma \in \operatorname{Stab}_{\Gamma}(\xi) \backslash \Gamma_{\xi}(d)} w_{T}\left(\gamma \gamma_{1}\right) & =\sum_{\gamma \in \operatorname{Stab}_{\Gamma}(\xi) \backslash \Gamma_{\xi}(d)} \int_{G / K} \chi_{B_{T}}(\xi \gamma g) \phi_{\epsilon}\left(\gamma_{1}^{-1} g\right) d \mu(g) \\
& =\int_{G / K} F_{T}^{\Gamma_{\xi}(d)}(g) \phi_{\epsilon}\left(\gamma_{1}^{-1} g\right) d \mu(g) \\
& =\int_{\Gamma_{\xi}(d) \backslash G / K} F_{T}^{\Gamma_{\xi}(d)}(g) \Phi_{\epsilon, \gamma_{1}}^{\Gamma_{\xi}(d)}(g) d \mu(g) .
\end{aligned}
$$

Thus

$$
\sum_{\gamma \in \operatorname{Stab}_{\Gamma}(\xi) \backslash \Gamma_{\xi}(d)} w_{T}\left(\gamma \gamma_{1}\right)=\left\langle F_{T}^{\Gamma_{\xi}(d)}, \Phi_{\epsilon, \gamma_{1}}^{\Gamma_{\xi}(d)}\right\rangle_{L^{2}\left(\Gamma_{\xi}(d) \backslash \mathbb{H}^{3}\right)}
$$

Denote by $\operatorname{Spec}\left(\Gamma \backslash \mathbb{H}^{3}\right)$ the spectrum of the Laplace operator on $L^{2}\left(\Gamma \backslash \mathbb{H}^{3}\right)$. As mentioned before, the work of Sullivan, generalizing Patterson's, implies that $\operatorname{Spec}\left(\Gamma \backslash \mathbb{H}^{3}\right) \cap[0,1) \neq \emptyset$, in which case $\lambda_{0}=\alpha(2-\alpha)$ is the base eigenvalue of $\Delta$. For the principal congruence subgroup

$$
\Gamma(d):=\{\gamma \in \Gamma: \gamma=I(\bmod d)\}
$$

of $\Gamma$ of level $d$, we have

$$
\operatorname{Spec}\left(\Gamma \backslash \mathbb{H}^{3}\right) \subset \operatorname{Spec}\left(\Gamma(d) \backslash \mathbb{H}^{3}\right) .
$$

The following is obtained by Bourgain, Gamburd and Sarnak in 4] and [5].

Theorem 8.6. Let $\Gamma$ be a Zariski dense subgroup of $\operatorname{Spin}_{Q}(\mathbb{Z})$ with $\delta_{\Gamma}>1$. Then there exist $1 \leq \theta<\delta_{\Gamma}$ such that we have for all square-free integers $d$,

$$
\operatorname{Spec}\left(\Gamma(d) \backslash \mathbb{H}^{3}\right) \cap\left[\theta(2-\theta), \delta_{\Gamma}\left(2-\delta_{\Gamma}\right)\right]=\left\{\delta_{\Gamma}\left(2-\delta_{\Gamma}\right)\right\} .
$$

In order to control the error term for $\left|\mathrm{A}_{d}(T)\right|$, we need a version of Corollary 7.3 uniform over all congruence subgroups $\Gamma_{\xi}(d)$ of $\Gamma$.

Proposition 8.7. There exists $\epsilon_{0}>0$, uniform over all square-free integers $d$, such that for any $\gamma_{1} \in \Gamma$ and for any congruence subgroup $\Gamma_{\xi}(d)$ of $\Gamma$, we have

$$
\left\langle F_{T}^{\Gamma_{\xi}(d)}, \Phi_{\epsilon, \gamma_{1}}^{\Gamma_{\xi}(d)}\right\rangle_{L^{2}\left(\Gamma_{\xi}(d) \backslash \mathbb{H}^{3}\right)}=\frac{c_{\phi_{0}} \cdot d_{\epsilon}}{\delta_{\Gamma} \cdot\left[\Gamma: \Gamma_{\xi}(d)\right]} \cdot\|\xi\|^{\alpha} \cdot T^{\alpha}+O\left(\epsilon^{-3} T^{\alpha-\epsilon_{0}}\right)
$$

for some $d_{\epsilon}>0$, where the implied constant depends only on $\Lambda_{N}(\Gamma)$.

Proof. Note that the congruence subgroup $\Gamma(d)$ of level $d$ is a finite index subgroup of $\Gamma_{\xi}(d)$. Since

$$
\operatorname{Spec}\left(\Gamma_{\xi}(d) \backslash \mathbb{H}^{3}\right) \subset \operatorname{Spec}\left(\Gamma(d) \backslash \mathbb{H}^{3}\right),
$$

the spectral gap Theorem 8.6 holds for the family $\Gamma_{\xi}(d), d$ square-free, as well.

As we are assuming $\|\cdot\|$ is $g_{0} K g_{0}^{-1}$-invariant, by Corollary 7.3, we have

$$
\left\langle F_{T}, \Phi_{\epsilon, \gamma_{1}}^{\Gamma_{\xi}(d)}\right\rangle=\left\langle\Phi_{\epsilon, \gamma_{1}}^{\Gamma_{\xi}(d)}, \phi_{0}^{\Gamma_{\xi}^{(d)}}\right\rangle \cdot \delta_{\Gamma_{\xi}(d)}^{-1} \cdot c_{\phi_{0}^{\Gamma_{\xi}(d)}} \cdot T^{\delta_{\Gamma}} \cdot\|\xi\|^{\alpha} \cdot\left(1+O\left(T^{-\epsilon_{0}}\right)\right),
$$

where $\epsilon_{0}$ depends only on the spectral gap for $L^{2}\left(\Gamma_{\xi}(d) \backslash \mathbb{H}^{3}\right)$ and the implied constant depends only on

$$
\Lambda_{N}\left(g_{0}^{-1} \Gamma_{\xi}(d) g_{0}\right)=\left\{\left[n_{x}\right] \in\left(N \cap g_{0}^{-1} \Gamma_{\xi}(d) g_{0}\right) \backslash N: x \in \Lambda\left(g_{0}^{-1} \Gamma_{\xi}(d) g_{0}\right)\right\} .
$$


As $\Gamma_{\xi}(d)$ is a subgroup of finite index in $\Gamma, \delta_{\Gamma_{\xi}(d)}=\delta_{\Gamma}$ and

$$
\Lambda\left(g_{0}^{-1} \Gamma_{\xi}(d) g_{0}\right)=\Lambda\left(g_{0}^{-1} \Gamma g_{0}\right),
$$

and moreover by the definition of $\Gamma_{\xi}(d)$, we have

$$
\operatorname{Stab}_{\Gamma}(\xi)=\operatorname{Stab}_{\Gamma_{\xi}(d)}(\xi)
$$

implying

$$
N \cap g_{0}^{-1} \Gamma_{\xi}(d) g_{0}=N \cap g_{0}^{-1} \Gamma g_{0} .
$$

Hence

$$
\Lambda_{N}\left(g_{0}^{-1} \Gamma_{\xi}(d) g_{0}\right)=\Lambda_{N}\left(g_{0}^{-1} \Gamma g_{0}\right),
$$

yielding that the implied constant in (8.8) is independent of $d$.

By Theorem 8.6 $\epsilon_{0}$ can be taken to be uniform over all $d$. If $\Gamma_{0}<\Gamma$ is a subgroup of finite index, the base eigenfunction in $L^{2}\left(\Gamma_{0} \backslash \mathbb{H}^{3}\right)$ with the unit $L^{2}$-norm is given by

with $\phi_{0}=\phi_{0}^{\Gamma}$. Therefore

$$
\phi_{0}^{\Gamma_{0}}:=\frac{1}{\sqrt{\left[\Gamma: \Gamma_{0}\right]}} \phi_{0}
$$

$$
\left\langle\Phi_{\epsilon, \gamma_{1}}^{\Gamma_{\xi}^{(d)}}, \phi_{0}^{\Gamma_{\xi}(d)}\right\rangle \cdot c_{\phi_{0}^{\Gamma_{\xi}(d)}}=\left\langle\Phi_{\epsilon, e}, \phi_{0}\right\rangle \cdot c_{\phi_{0}} \cdot \frac{1}{\left[\Gamma: \Gamma_{\xi}(d)\right]} .
$$

This finishes the proof as $\delta_{\Gamma}=\alpha$, by setting $d_{\epsilon}=\left\langle\Phi_{\epsilon, e}, \phi_{0}\right\rangle$.

Setting

$$
\mathcal{X}=\delta_{\Gamma}^{-1} \cdot c_{\phi_{0}} \cdot d_{\epsilon} \cdot\|\xi\|^{\alpha} \cdot T^{\alpha},
$$

the following corollary is immediate from Lemma 8.5 and Proposition 8.7

Corollary 8.9. There exists $\epsilon_{0}>0$ uniform over all square-free integers $d$ such that

where

$$
\left|\mathrm{A}_{d}(T)\right|=\mathcal{O}_{f}^{0}(d)\left(\frac{1}{\left[\Gamma: \Gamma_{\xi}(d)\right]} \mathcal{X}+O\left(\mathcal{X}^{1-\epsilon_{0}}\right)\right),
$$

$$
\mathcal{O}_{f}^{0}(d)=\sum_{\substack{\gamma \in \Gamma_{\xi}(d) \backslash \Gamma \\ f(\xi \gamma) \equiv 0(d)}} 1 .
$$

8.3. Proof of Theorem 1.4. We set

$$
f_{1}\left(x_{1}, x_{2}, x_{3}, x_{4}\right)=x_{1} \quad \text { and } \quad f_{2}\left(x_{1}, x_{2}, x_{3}, x_{4}\right)=x_{1} x_{2} .
$$

For $d$ square-free and $i=1,2$, set

$$
g_{i}(d)=\mathcal{O}_{f_{i}}^{0}(d) /\left[\Gamma: \Gamma_{\xi}(d)\right] .
$$

Proposition 8.10. There exists a finite set $S$ of primes such that:

(1) for any square-free integer $d=d_{1} d_{2}$ with no prime factors in $S$ and for each $i=1,2$,

$$
g_{i}\left(d_{1} d_{2}\right)=g_{i}\left(d_{1}\right) \cdot g_{i}\left(d_{2}\right)
$$

(2) for any prime $p$ outside $S$,

$$
g_{1}(p) \in(0,1) \quad \text { and } \quad g_{1}(p)=p^{-1}+O\left(p^{-3 / 2}\right) ;
$$

(3) for any prime $p$ outside $S$,

$$
g_{2}(p) \in(0,1) \quad \text { and } \quad g_{2}(p)=2 p^{-1}+O\left(p^{-3 / 2}\right) .
$$


Proof. According to the theorem of Matthews, Vaserstein and Weisfeiler [27, there exists a finite set of primes $S$ so that

- for all primes $p$ outside $S, \Gamma$ projects onto $G\left(\mathbb{F}_{p}\right)$;

- for $d=p_{1} \cdots p_{t}$ square-free with $p_{i} \notin S$, the diagonal reduction

$$
\Gamma \rightarrow G(\mathbb{Z} / d \mathbb{Z}) \rightarrow G\left(\mathbb{F}_{p_{1}}\right) \times \cdots \times G\left(\mathbb{F}_{p_{t}}\right)
$$

is surjective.

Enlarge $S$ so that the $G\left(\mathbb{F}_{p}\right)$ 's have no common composition factors for different $p$ 's outside $S$. This is possible because $G=\operatorname{Spin}(Q)$ can be realized as $\mathrm{SL}_{2}$ over $\mathbb{Q}[\sqrt{-1}]$. Hence there exists a finite set $S$ of primes such that for $p$ outside $S$,

$$
G\left(\mathbb{F}_{p}\right)=\left\{\begin{array}{lr}
\mathrm{SL}_{2}\left(\mathbb{F}_{p}\right) \times \mathrm{SL}_{2}\left(\mathbb{F}_{p}\right) & \text { for } p \equiv 1(4) \\
\mathrm{SL}_{2}\left(\mathbb{F}_{p^{2}}\right) & \text { for } p \equiv 3(4) .
\end{array}\right.
$$

It then follows from Goursat's lemma [23, p.75] that $\Gamma$ surjects onto $G\left(\mathbb{Z} / d_{1} \mathbb{Z}\right) \times$ $G\left(\mathbb{Z} / d_{2} \mathbb{Z}\right)$ for any square-free $d_{1}$ and $d_{2}$ with no prime factors in $S$. This implies that for $d=d_{1} d_{2}$ square-free and without any prime factors in $S$, the orbit of $\xi$ $\bmod d$, say $\mathcal{O}(d)$, is equal to $\mathcal{O}\left(d_{1}\right) \times \mathcal{O}\left(d_{2}\right)$ in $\left(\mathbb{Z} / d_{1} \mathbb{Z}\right)^{4} \times\left(\mathbb{Z} / d_{2} \mathbb{Z}\right)^{4}=(\mathbb{Z} / d \mathbb{Z})^{4}$. It also follows that $\mathcal{O}^{0}(d)$ is equal to $\mathcal{O}^{0}\left(d_{1}\right) \times \mathcal{O}^{0}\left(d_{2}\right)$. Therefore $g(d)=g\left(d_{1}\right) g\left(d_{2}\right)$, as desired.

Denote by $V$ the cone defined by $Q=0$ minus the origin, i.e.,

$$
V=\left\{\left(x_{1}, x_{2}, x_{3}, x_{4}\right) \neq 0: \sum_{i=1}^{4} 2 x_{i}^{2}-\left(\sum_{i=1}^{4} x_{i}\right)^{2}=0\right\} .
$$

Note that

$$
W_{1}:=\left\{x \in V: f_{1}(x)=0\right\}=\left\{\left(0, x_{2}, x_{3}, x_{4}\right) \neq 0: \sum_{i=2}^{4} 2 x_{i}^{2}-\left(\sum_{i=2}^{4} x_{i}\right)^{2}=0\right\} .
$$

Since both quadratic forms

$$
Q\left(x_{1}, x_{2}, x_{3}, x_{4}\right)=\sum_{i=1}^{4} 2 x_{i}^{2}-\left(\sum_{i=1}^{4} x_{i}\right)^{2} \text { and } Q\left(0, x_{2}, x_{3}, x_{4}\right)=\sum_{i=2}^{4} 2 x_{i}^{2}-\left(\sum_{i=2}^{4} x_{i}\right)^{2}
$$

are absolutely irreducible, we have by [3, Thm. 1.2.B],

$$
\# V\left(\mathbb{F}_{p}\right)=p^{3}+O\left(p^{5 / 2}\right), \quad \# W_{1}\left(\mathbb{F}_{p}\right)=p^{2}+O\left(p^{3 / 2}\right) .
$$

Since $V$ is a homogeneous space of $G$ with a connected stabilizer, by [35, Prop. $3.22]$,

$$
\mathcal{O}(p)=V\left(\mathbb{F}_{p}\right) \text {, and hence } \mathcal{O}_{f_{1}}^{0}(p)=W_{1}\left(\mathbb{F}_{p}\right) .
$$

Therefore we deduce $g_{1}(p)=\frac{\# \mathcal{O}_{f_{1}}^{0}(p)}{\# \mathcal{O}(p)}=p^{-1}+O\left(p^{-3 / 2}\right)$, proving (1).

Now $W_{2}:=\left\{x \in V: f_{2}(x)=0\right\}$ is the union of two quadrics given by $V \cap\left\{x_{1}=\right.$ $0\}$ and $V \cap\left\{x_{2}=0\right\}$. Hence

$$
\# W_{2}\left(\mathbb{F}_{p}\right)=2 p^{2}+O\left(p^{3 / 2}\right) .
$$

This yields that $g(p)=2 p^{-1}+O\left(p^{-3 / 2}\right)$. 
We are now ready to prove Theorem 1.4 First consider $f_{1}\left(x_{1}, x_{2}, x_{3}, x_{4}\right)=x_{1}$ so that $\mathrm{A}(T)=\left\{a_{n}(T)\right\}$, where

$$
a_{n}(T):=\sum_{\substack{\gamma \in \operatorname{Stab}_{\Gamma}(\xi) \backslash \Gamma \\ f_{1}(\xi \gamma)=n}} w_{T}(\gamma)
$$

is a smoothed count for the number of vectors $\left(x_{1}, x_{2}, x_{3}, x_{4}\right)$ in the orbit $\xi \mathcal{A}^{t}$ of max norm bounded above by $T$ and $x_{1}=n$.

By Lemma 8.9 and as $\# \mathcal{O}_{f_{1}}^{0}(d)$ is multiplicative with $\# \mathcal{O}_{f_{1}}^{0}(p)=p^{2}+O\left(p^{3 / 2}\right)$, the quantity $r_{d}(\mathrm{~A})$ in the decomposition (8.2) satisfies for some $\epsilon_{0}>0$,

$$
r_{d}(\mathrm{~A}) \ll d^{2} T^{\alpha-\epsilon_{0}} .
$$

Thus for any $\varepsilon_{1}>0$,

$$
\sum_{d<D, d \mid P} \tau_{3}(d)\left|r_{d}(\mathrm{~A})\right| \ll_{\varepsilon_{1}} D^{3+\varepsilon_{1}} T^{\alpha-\epsilon_{0}},
$$

which is $\ll T^{\alpha} / \log T$ for $D=T^{\epsilon_{0} / 4}$, say. The key here is that $D$ can be taken as large as a fixed power of $T$. Let $P$ be the product of all primes $p<D=T^{\epsilon_{0} / 4}$ outside of the bad set $S$.

As $h$ is a multiplicative function defined by $h(p)=\frac{g_{1}(p)}{1-g_{1}(p)}$ for primes $p$ with $g_{1}$ in Proposition 8.10, we deduce that (cf. [19, 6.6])

$$
\sum_{d<\sqrt{D}, d \mid P} h(d) \gg \log D \gg \log T,
$$

and Theorem 8.1 gives

$$
S(\mathrm{~A}(T), P) \ll T^{\alpha} / \log T \text {. }
$$

Therefore

$$
\begin{aligned}
& \#\left\{\left(x_{1}, x_{2}, x_{3}, x_{4}\right) \in \xi \mathcal{A}^{t}: \max _{1 \leq i \leq 4}\left|x_{i}\right|<T,\left(x_{1}, \prod_{p<T^{\epsilon_{0} / 4}} p\right)=1\right\} \\
& \ll S(\mathrm{~A}((1+\epsilon) T), P) \ll T^{\alpha} / \log T .
\end{aligned}
$$

Hence

$$
\#\left\{\left(x_{1}, x_{2}, x_{3}, x_{4}\right) \in \xi \mathcal{A}^{t}: \max _{1 \leq i \leq 4}\left|x_{i}\right|<T, x_{1}: \operatorname{prime}\right\} \ll T^{\alpha} / \log T .
$$

Since this argument is symmetric in the $x_{i}$ 's, we have

$$
\#\left\{\left(x_{1}, x_{2}, x_{3}, x_{4}\right) \in \xi \mathcal{A}^{t}: \max _{1 \leq i \leq 4}\left|x_{i}\right|=\text { prime at most } T\right\} \ll T^{\alpha} / \log T .
$$

By Lemma 2.5. this proves that

$$
\pi^{\mathcal{P}}(T) \ll \frac{T^{\alpha}}{\log T} .
$$

In order to prove

$$
\pi_{2}^{\mathcal{P}}(T) \ll \frac{T^{\alpha}}{(\log T)^{2}},
$$

we proceed the same way with the polynomial $f_{2}\left(x_{1}, x_{2}, x_{3}, x_{4}\right)=x_{1} x_{2}$ and with the sequence $\mathrm{A}(T)=\left\{a_{n}(T)\right\}$, where

$$
a_{n}(T):=\sum_{\substack{\gamma \in \operatorname{Stab} \Gamma(\xi) \backslash \Gamma \\ f_{2}(\xi \gamma)=n}} w_{T}(\gamma)
$$


is a smoothed count for the number of vectors $\left(x_{1}, x_{2}, x_{3}, x_{4}\right)$ in the orbit $\xi \mathcal{A}^{t}$ of max norm bounded above by $T$ and $x_{1} x_{2}=n$.

Note that $g_{2}(p)=2 p^{-1}+O\left(p^{-3 / 2}\right)$ by Proposition 8.10 and that

$$
\sum_{d<\sqrt{D}, d \mid P} h(d) \gg(\log D)^{2} \gg(\log T)^{2}
$$

for $h(p)=\frac{g_{2}(p)}{1-g_{2}(p)}$ for primes $p$.

Therefore Theorem 8.1 gives

$$
S(\mathrm{~A}(T), P) \ll T^{\alpha} /(\log T)^{2},
$$

which implies that

$$
\#\left\{\left(x_{1}, x_{2}, x_{3}, x_{4}\right) \in \xi \mathcal{A}^{t}: \max _{1 \leq i \leq 4}\left|x_{i}\right|<T, x_{1}, x_{2}: \text { primes }\right\} \ll T^{\alpha} /(\log T)^{2} .
$$

Again by the symmetric property of the $x_{i}$ 's, we have

$$
\#\left\{x \in \xi \mathcal{A}^{t}: \max _{1 \leq i \leq 4}\left|x_{i}\right|<T, x_{i}, x_{j} \text { : primes for some } i \neq j\right\} \ll T^{\alpha} /(\log T)^{2} .
$$

By Lemma 2.5, this proves

$$
\pi_{2}^{\mathcal{P}}(T) \ll T^{\alpha} /(\log T)^{2} .
$$

\section{ACKNOWLEDGMENTS}

We are grateful to Peter Sarnak for introducing us to this problem and for helpful discussions. We also thank Yves Benoist, Jeff Brock and Curt McMullen for useful conversations.

\section{Appendix. Non-ACCumulation of EXPANDing ClOSED hOROSPHERES ON singular tubes (by Hee Oh and Nimish Shah)}

In this appendix, we deduce Theorem A.1 from Theorem 6.1 Recall that $\Gamma<$ $G=\mathrm{PSL}_{2}(\mathbb{C})$ is a torsion-free discrete geometrically finite group with $\delta_{\Gamma}>1$ and that $\phi_{0} \in L^{2}\left(\Gamma \backslash \mathbb{H}^{3}\right)$ denotes the positive base eigenfunction of $\Delta$ of eigenvalue $\delta_{\Gamma}\left(2-\delta_{\Gamma}\right)$ and of norm $\int_{\Gamma \backslash \mathbb{H}^{3}} \phi_{0}^{2}(g) d \mu(g)=1$.

We continue the notation $N, a_{y}, N^{-}$, etc., from section 6 . We assume that $\Gamma \backslash \Gamma N$ is closed. By Corollary 4.13, for some $c_{\phi_{0}}>0$ and $d_{\phi_{0}} \geq 0$,

$$
\int_{\Gamma \backslash \Gamma N} \phi_{0}\left(n a_{y}\right) d n=c_{\phi_{0}} y^{2-\delta_{\Gamma}}+d_{\phi_{0}} y^{\delta_{\Gamma}} .
$$

We also recall the Burger-Roblin measure $\hat{\mu}$ defined in Theorem 6.10 which is normalized so that $\hat{\mu}\left(\phi_{0}\right)=1$.

Theorem A.1. For $\psi \in C_{c}\left(\mathrm{~T}^{1}\left(\Gamma \backslash \mathbb{H}^{3}\right)\right)$,

$$
\int_{n_{x} \in(N \cap \Gamma) \backslash N} \psi\left(n_{x} a_{y}\right) d x \sim c_{\phi_{0}} \cdot \hat{\mu}(\psi) \cdot y^{2-\delta_{\Gamma}} \quad \text { as } y \rightarrow 0 .
$$

Proof. The idea of the proof is motivated by the proof of Lemma 2.1 in [37. For each $0<y \leq 1$, define the measure $\mu_{y}$ on $\Gamma \backslash G / M=\mathrm{T}^{1}\left(\Gamma \backslash \mathbb{H}^{3}\right)$ by

$$
\mu_{y}(\psi)=c_{\phi_{0}}^{-1} y^{-2+\delta_{\Gamma}} \int_{(N \cap \Gamma) \backslash N} \psi\left(\Gamma \backslash \Gamma\left[n_{x} a_{y}\right]\right) d x
$$

for $\psi \in C_{c}(\Gamma \backslash G / M)$. 
Consider the family

$$
\mathcal{M}:=\left\{\mu_{y}: 0<y<1\right\} .
$$

We claim that $\mathcal{M}$ is relatively compact in the set of locally finite Borel measures on $\Gamma \backslash G / M$ with respect to the weak*-topology. For any compact subset $C \subset$ $\Gamma \backslash G / M$, let $\psi$ be a $K$-invariant smooth non-negative function of compact support which is one over $C$. Then

$$
\mu_{y}(C) \leq \mu_{y}(\psi) .
$$

As $\mu_{y}(\psi) \rightarrow \hat{\mu}(\psi)=\left\langle\psi, \phi_{0}\right\rangle$ by Theorem 6.1, the claim follows.

It now suffices to show that every accumulation point of $\mathcal{M}$ is equal to $\hat{\mu}$. Let $\mu_{0}$ be an accumulation point of $\mathcal{M}$, which is clearly $N$-invariant.

We denote by $\mathcal{E}_{P}$ the set of vectors $v \in \mathrm{T}^{1}\left(\Gamma \backslash \mathbb{H}^{3}\right)$, the horospheres determined by which are closed. In the identification of $\mathrm{T}^{1}\left(\Gamma \backslash \mathbb{H}^{3}\right)$ with $\Gamma \backslash G / M$, the set $\mathcal{E}_{P}$ corresponds to the image under the projection $\Gamma \backslash G \rightarrow \Gamma \backslash G / M$ of the sets $\Gamma \backslash \Gamma g N A$ for $\Gamma \backslash \Gamma g N$ closed.

Fix a bounded parabolic fixed point $\xi_{0}$ of $\Gamma$. If $\xi_{0}=g_{0}(\infty)$ for $g_{0} \in \mathrm{PSL}_{2}(\mathbb{C})$, then a cusp, say, $D\left(\xi_{0}\right)$, at $\xi_{0}$ is the image of $\bigcup_{y>y_{1}} g_{0} N a_{y}$ for some $y_{1} \gg 1$ under the projection $\pi: G \rightarrow \Gamma \backslash G / M$.

There exists $c_{0}>1$ such that for any $z=\Gamma \backslash \Gamma g_{0} n a_{y} \in D\left(\xi_{0}\right)$,

$$
c_{0}^{-1} y^{r-\delta} \leq \phi_{0}(z) \leq c_{0} y^{r-\delta},
$$

where $r \in\{1,2\}$ is the rank of $\xi_{0}$ (see [46, Sec. 5] as well as the proof of [8, Lem. $3.5])$.

Noting that $D_{0}=D_{0}\left(\xi_{0}\right) \subset \mathcal{E}_{P}$, we first claim that for any weak limit, say, $\mu_{0}$, of $\mathcal{M}$,

$$
\mu_{0}\left(D_{0}\right)=0 .
$$

Let $Q$ be a relatively compact open subset of $D_{0}$. For $y_{\epsilon}>1$, setting $D_{\epsilon}:=$ $D_{0} a_{y_{\epsilon}} \subset D_{0}$, we have that

$$
\int_{D_{\epsilon} K} \phi_{0}^{2} d \mu(g) \ll y_{\epsilon}^{r-2 \delta} .
$$

For the part of $D_{\epsilon} K$ inside the unit neighborhood of the convex core of $\Gamma$, this estimate follows from the proof of [8, Lem. 4.2]. When $r=1$, the integral of $\phi_{0}^{2}$ over the part of $D_{\epsilon} K$ outside the unit neighborhood of the convex core of $\Gamma$ is comparable to

$$
\int_{\log y_{\epsilon}}^{\infty} \int_{x=e^{t}}^{\infty} e^{-2 \delta t} d x d t \asymp y_{\epsilon}^{1-2 \delta}
$$

Fixing a neighborhood $U$ of $e$ in $N^{-} M$ such that $D_{0} U \subset D_{0} K$, the set $D_{\epsilon} U a_{y_{\epsilon}}^{-1}$ is a neighborhood of $Q$. Therefore if $\mu_{y_{i}}$ weakly converges to $\mu_{0}$,

$$
\begin{aligned}
c_{\phi_{0}} \cdot \mu_{0}\left(\phi_{0} \cdot \chi_{Q}\right) & \leq \lim _{y_{i} \rightarrow 0} \frac{1}{y_{i}^{2-\delta}} \int_{\Gamma \backslash \Gamma N}\left(\phi_{0} \cdot \chi_{D_{\epsilon} U a_{y_{\epsilon}}^{-1}}\right)\left(n a_{y_{i}}\right) d n \\
& =\lim _{y_{i} \rightarrow 0} \frac{1}{y_{i}^{2-\delta}} \int_{\Gamma \backslash \Gamma N} \phi_{0}\left(n a_{y_{i}}\right) \cdot \chi_{D_{\epsilon} U}\left(n a_{y_{i}} a_{y_{\epsilon}}\right) d n .
\end{aligned}
$$

As $y_{\epsilon}>1$ and $U \subset N^{-} M$,

$$
a_{y_{\epsilon}} U a_{y_{\epsilon}^{-1}} \subset U \text {. }
$$

Hence if $n a_{y y_{\epsilon}} \in D_{\epsilon} U$, then

$$
n a_{y} \in D_{\epsilon} a_{y_{\epsilon}^{-1}}\left(a_{y_{\epsilon}} U a_{y_{\epsilon}^{-1}}\right) \subset D_{\epsilon} a_{y_{\epsilon}^{-1}} U \subset D_{0} U \text {. }
$$


Moreover if $n a_{y y_{\epsilon}} \in \Gamma g_{0} n^{\prime} a_{y^{\prime}} M U$ for some $n^{\prime} \in N$ and $y^{\prime}>y_{1}$, then

$$
n a_{y} \in \Gamma g_{0} n^{\prime} a_{y^{\prime}} a_{y_{\epsilon}}^{-1}\left(a_{y_{\epsilon}} M U a_{y_{\epsilon}}^{-1}\right) \subset g_{0} n^{\prime} a_{y^{\prime} y_{\epsilon}^{-1}} M U .
$$

Using the formula

$$
\phi_{0}(g)=\int_{\xi \in \Lambda(\Gamma)} e^{-\delta \beta_{\xi}(g o, o)} d \nu_{0}(\xi)
$$

for $g \in G$ (see (4.2)), it is easy to check that

$$
e^{-\delta d(u o, o)} \phi_{0}(g) \leq \phi_{0}(g u) \leq e^{\delta d(u o, o)} \phi_{0}(g)
$$

for any $g, u \in G$. Therefore we can deduce from (A.2) and (A.5) that for some constant $c^{\prime}>1$

$$
\phi_{0}\left(n a_{y}\right) \leq c^{\prime} \cdot y_{\epsilon}^{\delta-r} \cdot \phi_{0}\left(n a_{y} a_{y_{\epsilon}}\right)
$$

for all $n a_{y} a_{y_{\epsilon}} \in D_{\epsilon} U$. Hence

$$
\begin{aligned}
c_{\phi_{0}} \cdot & \mu_{0}\left(\phi_{0} \cdot \chi_{Q}\right) \\
& \leq c_{\phi_{0}} c^{\prime} y_{\epsilon}{ }^{2-r} \lim _{y_{i} \rightarrow 0} \frac{1}{\left(y_{i} y_{\epsilon}\right)^{2-\delta}} \int_{\Gamma \backslash \Gamma N}\left(\phi_{0} \cdot \chi_{D_{\epsilon} U}\right)\left(\Gamma \backslash \Gamma n a_{y_{i}} a_{y_{\epsilon}}\right) d n \\
& \leq c_{\phi_{0}} c^{\prime} y_{\epsilon}^{2-r} \int_{\Gamma \backslash \mathbb{H}^{3}}\left(\phi_{0}^{2} \cdot \chi_{D_{\epsilon} K}\right)(g) d \mu(g) \text { by Theorem } 6.1 \\
& \ll y_{\epsilon}^{2-2 \delta} \text { by }
\end{aligned}
$$

Therefore

$$
\mu_{0}\left(\phi_{0} \cdot \chi_{Q}\right) \ll y_{\epsilon}^{2-2 \delta} .
$$

As $\delta>1, y_{\epsilon}>1$ is arbitrary, and $\min _{g \in Q} \phi_{0}(g)>0$, we have $\mu_{0}(Q)=0$. This proves the claim A.3.

We now claim that $\mu_{0}\left(\mathcal{E}_{P}\right)=0$ for any weak limit $\mu_{0}: \mu_{y_{i}} \rightarrow \mu_{0}$. Suppose not. Since there are only finitely many cusps, there exists a relatively compact open subset $Q \subset \mathcal{E}_{P}$ and a bounded parabolic fixed point $\xi_{0} \in \Lambda(\Gamma)$ such that $\mu_{0}(Q)>0$ and its image $a_{y}(Q)=Q a_{y}$ under the geodesic flow converges to $\xi_{0}$ as $y \rightarrow \infty$. Fix $y_{0}>1$ such that

$$
Q a_{y_{0}} \subset D_{0}\left(\xi_{0}\right)
$$

By passing to a subsequence, we may assume that $\mu_{y_{i} y_{0}}$ is convergent with a weak limit, say, $\mu_{0}^{\prime}$. Since $Q a_{y_{0}} \subset D_{0}\left(\xi_{0}\right)$, by (A.3), we have

$$
\mu_{0}^{\prime}\left(Q a_{y_{0}}\right)=0 \text {. }
$$

Therefore for any $\epsilon>0$, there exists a neighborhood $U_{\epsilon} \subset N^{-} M$ of $e$ such that

$$
\mu_{0}^{\prime}\left(Q a_{y_{0}} U_{\epsilon}\right)<y_{0}^{-2+\delta} \epsilon .
$$

Noting that $Q a_{y_{0}} U_{\epsilon} a_{y_{0}}^{-1}$ is a neighborhood of $Q$, we have

$$
\begin{aligned}
c_{\phi_{0}} \cdot \mu_{0}(Q) & \leq \lim _{y_{i} \rightarrow 0} \frac{1}{y_{i}^{2-\delta}} \int_{\Gamma \backslash \Gamma N} \chi_{Q a_{y_{0}} U_{\epsilon} a_{y_{0}}^{-1}}\left(n a_{y_{i}}\right) d n \\
& =y_{0}^{2-\delta} \lim _{y_{i} \rightarrow 0} \frac{1}{\left(y_{0} y_{i}\right)^{2-\delta}} \int_{\Gamma \backslash \Gamma N} \chi_{Q a_{y_{0}} U_{\epsilon}}\left(n a_{y_{i}} a_{y_{0}}\right) d n \\
& =y_{0}^{2-\delta} \mu_{0}^{\prime}\left(Q a_{y_{0}} U_{\epsilon}\right) \\
& \leq \epsilon \text { by (A.6). }
\end{aligned}
$$


Since $\epsilon>0$ is arbitrary, $\mu_{0}(Q)=0$. This proves

$$
\mu_{0}\left(\mathcal{E}_{P}\right)=0 .
$$

We deduce from Theorem 6.9 that

$$
\mu_{0}=\alpha_{1} \hat{\mu}
$$

for some $\alpha_{1} \geq 0$. On the other hand, by Theorem 6.1

$$
\mu_{0}\left(\phi_{0}\right)=\hat{\mu}\left(\phi_{0}\right)=1 \text {. }
$$

It follows that $\alpha_{1}=1$.

\section{REFERENCES}

[1] Thierry Aubin. Nonlinear analysis on manifolds. Monge-Ampère equations, volume 252 of Grundlehren der Mathematischen Wissenschaften [Fundamental Principles of Mathematical Sciences]. Springer-Verlag, New York, 1982. MR681859 (85j:58002)

[2] Alan F. Beardon and Bernard Maskit. Limit points of Kleinian groups and finite sided fundamental polyhedra. Acta Math., 132:1-12, 1974. MR0333164 (48:11489)

[3] A. I. Borevich and I. R. Shafarevich. Number theory. Translated from the Russian by Newcomb Greenleaf. Pure and Applied Mathematics, Vol. 20. Academic Press, New York, 1966. MR0195803 (33:4001)

[4] Jean Bourgain, Alex Gamburd, and Peter Sarnak. Affine linear sieve, expanders and sumproduct, Inventiones Math., 179: 559-644, 2010. MR2587341

[5] Jean Bourgain, Alex Gamburd, and Peter Sarnak. Generalization of Selberg's theorem and Selberg's sieve, 2009. Preprint.

[6] David W. Boyd. The sequence of radii of the Apollonian packing. Math. Comp., 39(159):249254, 1982. MR658230 (83i:52013)

[7] Marc Burger. Horocycle flow on geometrically finite surfaces. Duke Math. J., 61(3):779-803, 1990. MR 1084459 (91k:58102)

[8] Kevin Corlette and Alessandra Iozzi. Limit sets of discrete groups of isometries of exotic hyperbolic spaces. Trans. Amer. Math. Soc., 351(4):1507-1530, 1999. MR1458321|(99f:58122)

[9] H. S. M. Coxeter. The problem of Apollonius. Amer. Math. Monthly, 75:5-15, 1968. MR0230204 (37:5767)

[10] F. Dal'bo. Topologie du feuilletage fortement stable. Ann. Inst. Fourier (Grenoble), 50(3):981-993, 2000. MR:1779902 (2001i:37045)

[11] S. G. Dani. Invariant measures and minimal sets of horospherical flows. Invent. Math., 64(2):357-385, 1981. MR629475 (83c:22009)

[12] Alex Eskin and C. T. McMullen. Mixing, counting, and equidistribution in Lie groups. Duke Math. J., 71(1):181-209, 1993. MR.1230290 (95b:22025)

[13] E. Fuchs. Ph.D. Thesis, Princeton University, 2010.

[14] Ramesh Gangolli and V. S. Varadarajan. Harmonic analysis of spherical functions on real reductive groups, volume 101 of Ergebnisse der Mathematik und ihrer Grenzgebiete [Results in Mathematics and Related Areas]. Springer-Verlag, Berlin, 1988. MR.954385 (89m:22015)

[15] Ronald L. Graham, Jeffrey C. Lagarias, Colin L. Mallows, Allan R. Wilks, and Catherine H. Yan. Apollonian circle packings: number theory. J. Number Theory, 100(1):1-45, 2003. MR1971245 (2004d:11055)

[16] Ronald L. Graham, Jeffrey C. Lagarias, Colin L. Mallows, Allan R. Wilks, and Catherine H. Yan. Apollonian circle packings: geometry and group theory. I. The Apollonian group. Discrete Comput. Geom., 34(4):547-585, 2005. MR2173929 (2009a:11090a)

[17] Y. Guivarc'h and A. Raugi. Products of random matrices: convergence theorems. In Random matrices and their applications (Brunswick, Maine, 1984), volume 50 of Contemp. Math., pages 31-54. Amer. Math. Soc., Providence, RI, 1986. MR841080 (87m:60024)

[18] K. E. Hirst. The Apollonian packing of circles. J. London Math. Soc., 42:281-291, 1967. MR0209981(35:876) 
[19] Henryk Iwaniec and Emmanuel Kowalski. Analytic number theory, volume 53 of American Mathematical Society Colloquium Publications. American Mathematical Society, Providence, RI, 2004. MR2061214 (2005h:11005)

[20] Michael Kapovich. Hyperbolic manifolds and discrete groups, volume 183 of Progress in Mathematics. Birkhäuser Boston Inc., Boston, MA, 2001. MR.1792613 (2002m:57018)

[21] Alex Kontorovich. The hyperbolic lattice point count in infinite volume with applications to sieves. Duke Math. J., 149(1):1-36, 2009. MR.2541126

[22] Alex Kontorovich and Hee Oh. Almost prime Pythagorean triples in thin orbits. Preprint, 2009, arXiv:1001.0370.

[23] Serge Lang. Algebra, volume 211 of Graduate Texts in Mathematics. Springer-Verlag, New York, third edition, 2002. MR1878556 (2003e:00003)

[24] Peter D. Lax and Ralph S. Phillips. The asymptotic distribution of lattice points in Euclidean and non-Euclidean spaces. J. Funct. Anal., 46(3):280-350, 1982. MR661875 (83j:10057)

[25] Gregory Margulis. On some aspects of the theory of Anosov systems. Springer Monographs in Mathematics. Springer-Verlag, Berlin, 2004. With a survey by Richard Sharp: Periodic orbits of hyperbolic flows, Translated from the Russian by Valentina Vladimirovna Szulikowska. MR2035655 (2004m:37049)

[26] Bernard Maskit. Kleinian groups, volume 287 of Grundlehren der Mathematischen Wissenschaften [Fundamental Principles of Mathematical Sciences]. Springer-Verlag, Berlin, 1988. MR959135 (90a:30132)

[27] C. Matthews, L. Vaserstein, and B. Weisfeiler. Congruence properties of Zariski-dense subgroups. Proc. London Math. Soc, 48:514-532, 1984. MR735226 (85d:20040)

[28] F. Maucourant. Homogeneous asymptotic limits of Haar measures of semisimple linear groups and their lattices. Duke Math. J., 136(2):357-399, 2007. MR2286635 (2007j:22022)

[29] C. T. McMullen. Hausdorff dimension and conformal dynamics. III. Computation of dimension. Amer. J. Math., 120(4):691-721, 1998. MR.1637951 (2000d:37055)

[30] Amir Mohammadi and Alireza Salehi Golsefidy. Translates of horospherical measures and counting problems. Preprint, 2008.

[31] Hee Oh. Dynamics on Geometrically finite hyperbolic manifolds with applications to Apollonian circle packings and beyond. Proc. of ICM (Hyderabad, 2010).

[32] Hee Oh and Nimish Shah. Equidistribution and counting for orbits of geometrically finite hyperbolic groups. Preprint, arXiv:1001.2096.

[33] Hee Oh and Nimish Shah. The asymptotic distribution of circles in the orbits of Kleinian groups. Preprint, arXiv:1004.2130.

[34] S.J. Patterson. The limit set of a Fuchsian group. Acta Mathematica, 136:241-273, 1976. MR0450547(56:8841)

[35] Vladimir Platonov and Andrei Rapinchuk. Algebraic groups and number theory, volume 139 of Pure and Applied Mathematics. Academic Press Inc., Boston, MA, 1994. Translated from the 1991 Russian original by Rachel Rowen. MR.1278263 (95b:11039)

[36] Marina Ratner. On Raghunathan's measure conjecture. Ann. of Math. (2), 134(3):545-607, 1991. MR1135878 (93a:22009)

[37] Marina Ratner. Raghunathan's topological conjecture and distributions of unipotent flows. Duke Math. J., 63(1):235-280, 1991. MR1106945 (93f:22012)

[38] Thomas Roblin. Ergodicité et équidistribution en courbure négative. Mém. Soc. Math. Fr. (N.S.), (95):vi+96, 2003. MR2057305 (2005d:37060)

[39] Peter Sarnak. Asymptotic behavior of periodic orbits of the horocycle flow and Eisenstein series. Comm. Pure Appl. Math., 34(6):719-739, 1981. MR634284(83m:58060)

[40] Peter Sarnak. Letter to J. Lagarias, 2007. available at www.math.princeton.edu/ sarnak.

[41] Barbara Schapira. Equidistribution of the horocycles of a geometrically finite surface. Int. Math. Res. Not., (40):2447-2471, 2005. MR.2180113 (2006i:37073)

[42] Yehuda Shalom. Rigidity, unitary representations of semisimple groups, and fundamental groups of manifolds with rank one transformation group. Ann. of Math. (2), 152(1):113-182, 2000. MR1792293 (2001m:22022)

[43] F. Soddy. The bowl of integers and the hexlet. Nature, 139:77-79, 1936.

[44] F. Soddy. The kiss precise. Nature, 137:1021, 1937.

[45] Dennis Sullivan. The density at infinity of a discrete group of hyperbolic motions. Inst. Hautes Études Sci. Publ. Math., (50):171-202, 1979. MR.556586 (81b:58031) 
[46] Dennis Sullivan. Entropy, Hausdorff measures old and new, and limit sets of geometrically finite Kleinian groups. Acta Math., 153(3-4):259-277, 1984. MR766265 (86c:58093)

[47] William Thurston. The Geometry and Topology of Three-Manifolds. available at www.msri.org/publications/books. Electronic version-March 2002.

[48] J. B. Wilker. Sizing up a solid packing. Period. Math. Hungar., 8(2):117-134, 1977. MR0641055 (58:30759)

Department of Mathematics, Brown University, Providence, Rhode Island 02912

E-mail address: alexk@math.brown.edu

Current address: Department of Mathematics, Stony Brook University, Stony Brook, New York 11794-3651

E-mail address: alexk@math.sunysb.edu

Department of Mathematics, Brown University, Providence, Rhode Island 02912 and Department of Mathematics, Korea Institute for Advanced Study, Seoul, Korea

E-mail address: heeoh@math.brown.edu

Department of Mathematics, The Ohio State University, Columbus, Ohio 43210

E-mail address: shah@math.ohio-state.edu 